\title{
Biodiversitätsmuseum Göttingen
}

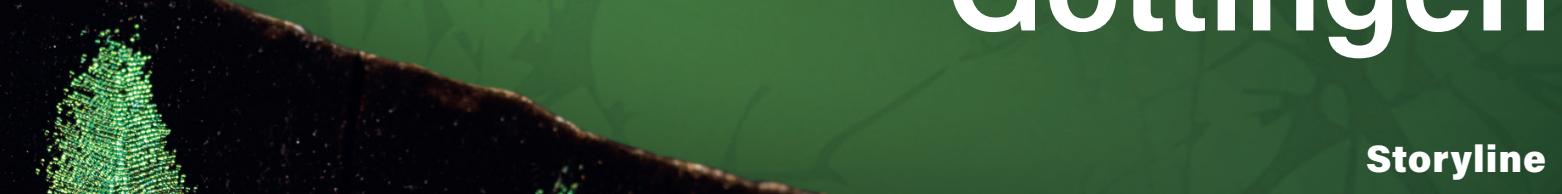

(1)
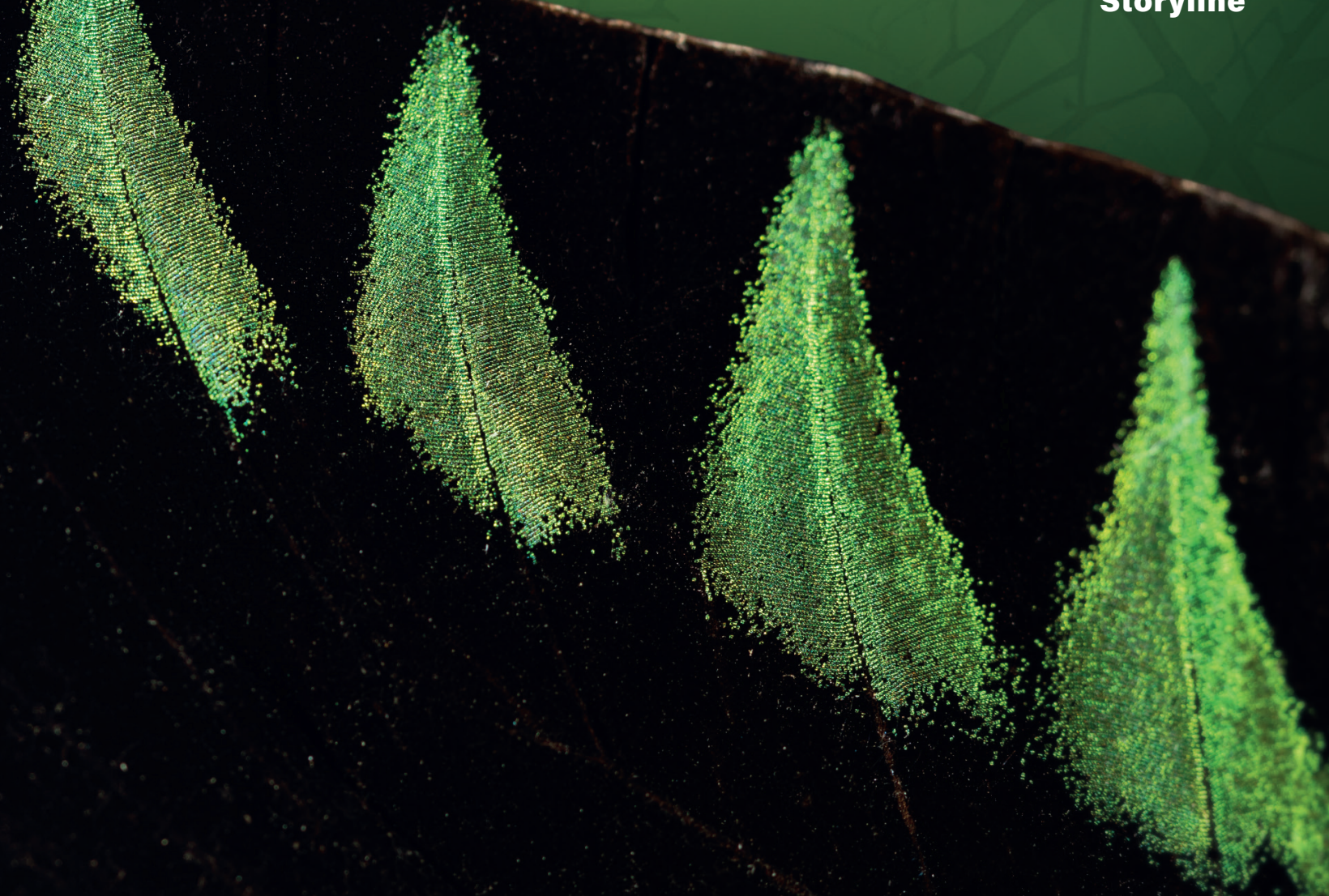


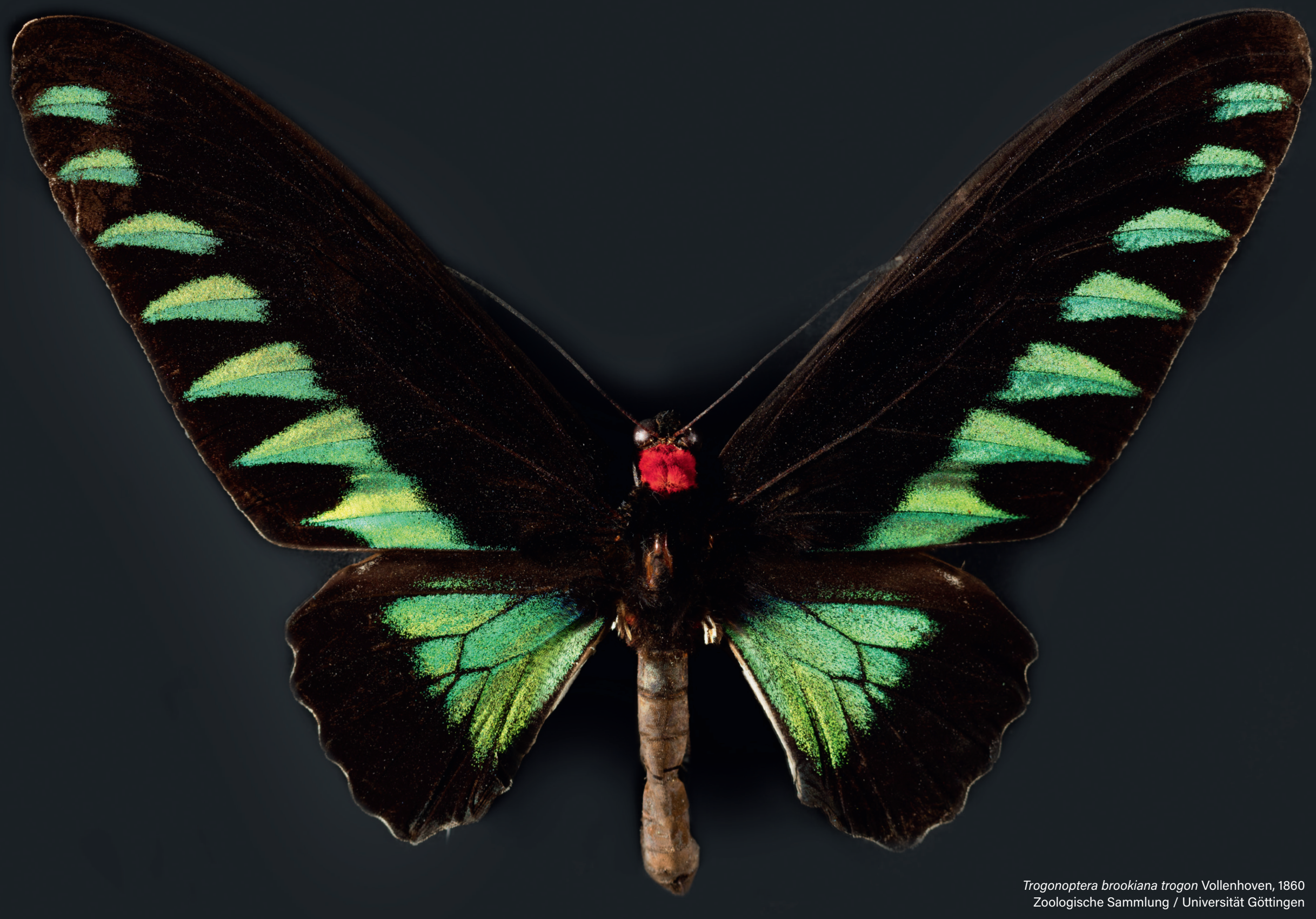




\section{Biodiversitätsmuseum Göttingen}

\section{Konzept und Storyline}

Biediversitätsmuseum

Vorgestellt von der Georg-August-Universität Göttingen

und Kunstraum GfK mbH - vivid exhibitions 


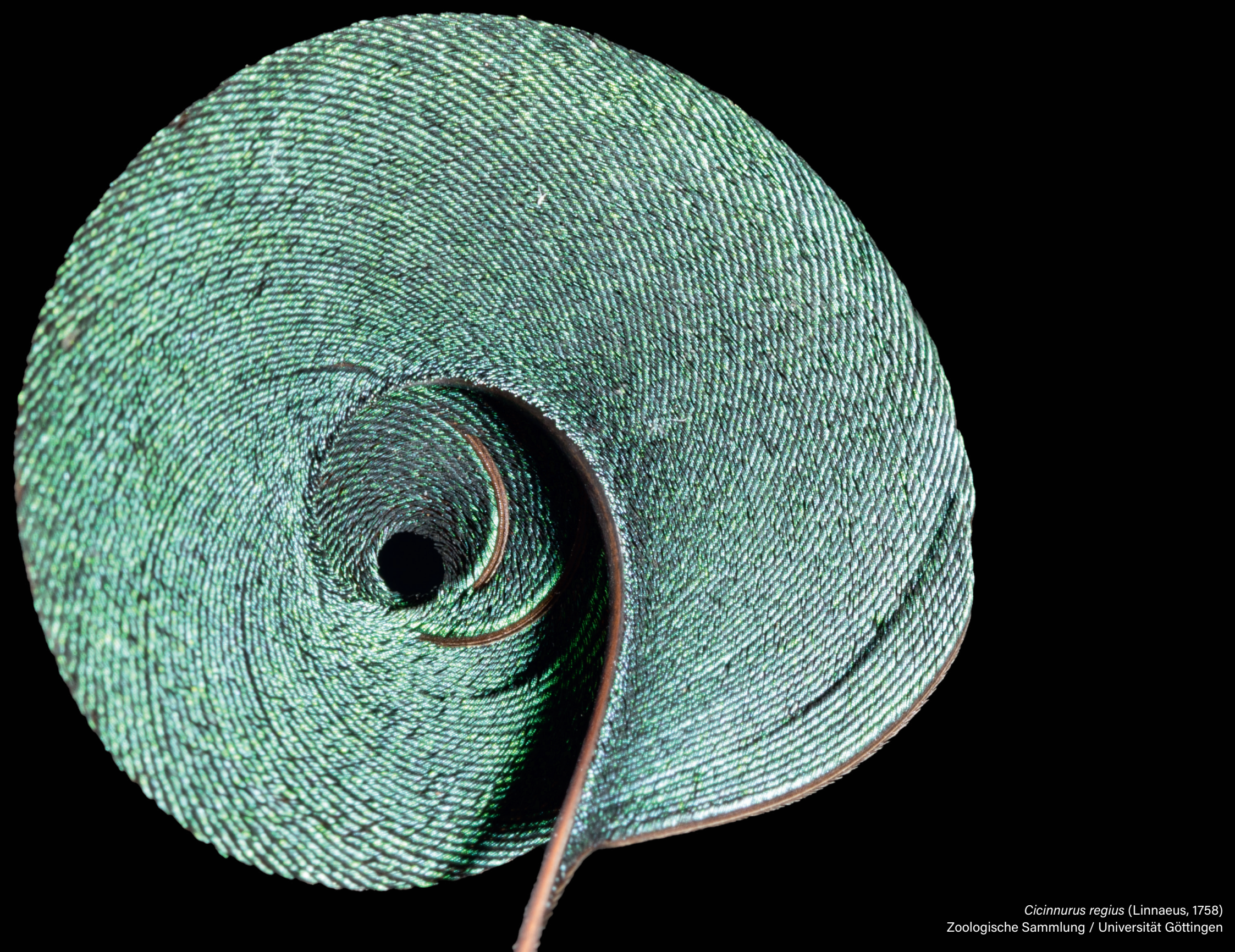


«Hier ist eine Fülle von Leben versammelt, die über einen Zeitraum von einer Milliarde Jahren entstanden ist. Diese Vielfalt hat Stürme überdauert sie in ihre Gene aufgenommen - und die Welt geschaffen, die uns geschaffen hat. Sie hält die Welt im Gleichgewicht. Wir sollten jedes bisschen der Biodiversität als unbezahlbar bewahren, während wir lernen, sie zu nutzen, und uns bewusst werden, was sie für die Menschheit bedeutet.»

- Edward O. Wilson 


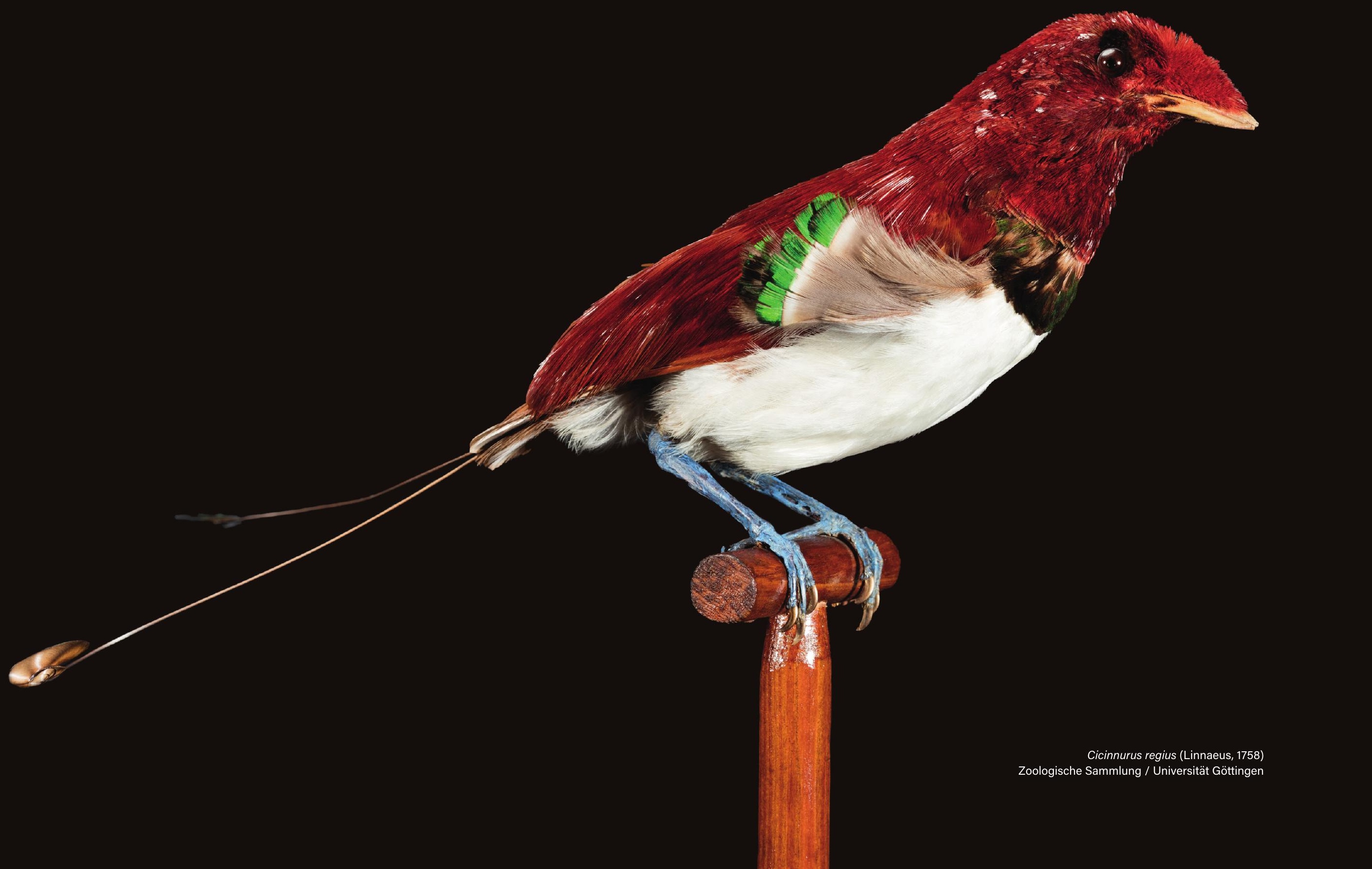




\section{Inhalt}

1. Unser Vorhaben

a. Biodiversität

b. Das Ziel 10

c. Das Museum 12

d. Unsere Intention 14

e. Die Themen 16

f. Die Zielgruppen 18

g. Die Sammlungen 20

h. Kommunikation \& Didaktik 22

2. Das Konzept 25

a. Wissenslücken \& wie man sie füllt 26

b. Die Dramaturgie 28

c. Die räumliche Organisation 30

d. Die Szenografie 32

e. Die Organisation der Information 34

f. Die Fensterelemente 36

g. Das Gefühl des Staunens 38

h. Wissenschaftliche Inhalte $\quad 40$

3. Gang durch die Ausstellung 43

- Prolog - Was ist Biodiversität?

Kapitel 1 - Was ist ein Ökosystem? 47

- Kapitel 2 - Was ist eine Art?

- Kapitel 3 - Was ist Evolution? 67

- Kapitel 4 - Warum ist Biodiversität wichtig? 77

- Kapitel 5 - Der Einfluss des Menschen 87

- Kapitel 6 - Entdecke Biodiversität in deiner Umgebung 97

- Epilog - Was kann ich tun? 105

4. Das Team 


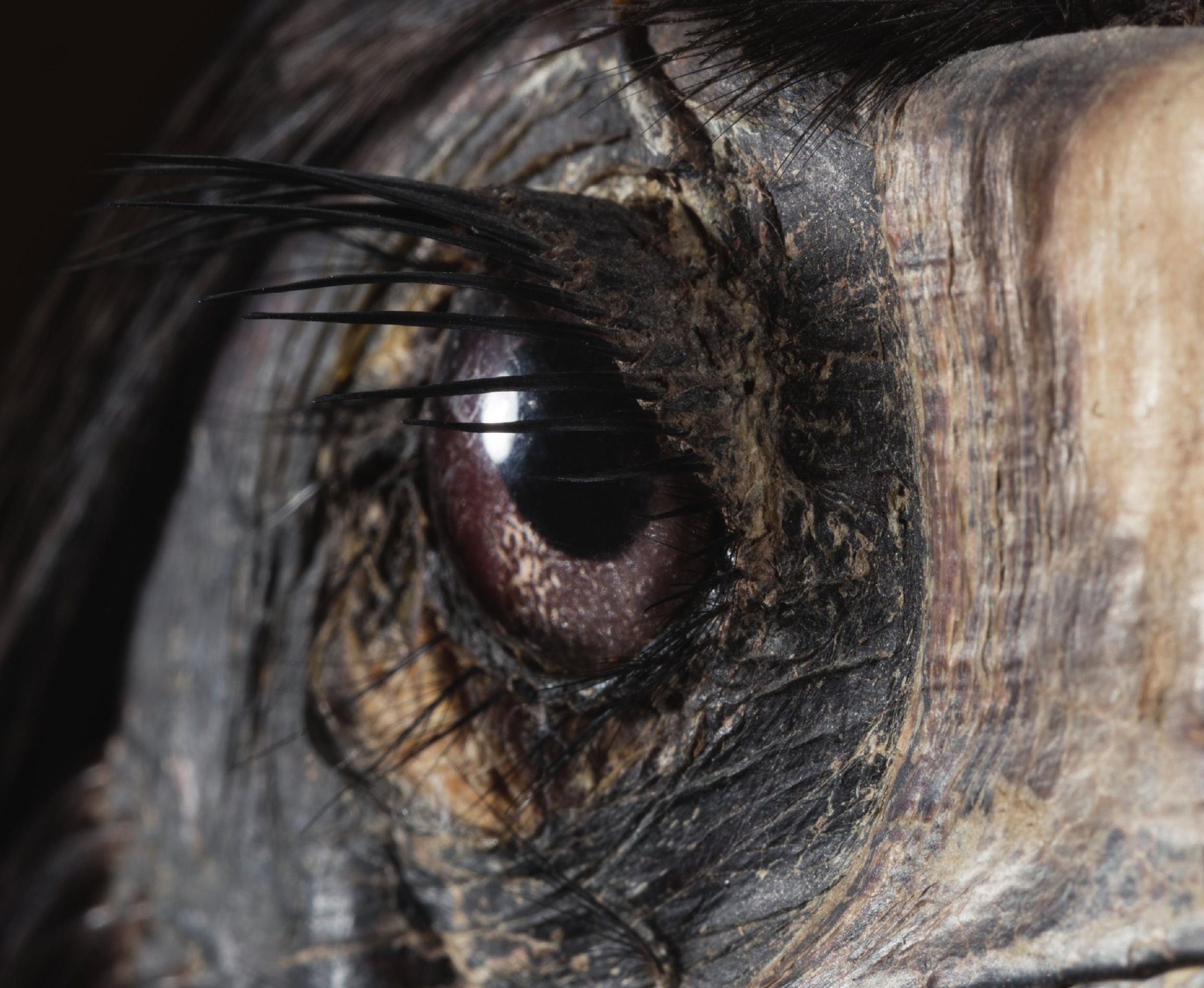


«Wenn man den Wert allen Lebens erkennt, verweilt man weniger in der Vergangenheit und konzentriert sich mehr auf die Erhaltung der Zukunft,»

- Dian Fossey

\section{Unser Vorhaben}




\section{Biodiversität}

Die Vielfalt des Lebens auf der Erde, in all seinen Formen und Interaktionen. Menschen und ihre unterschiedlichen Kulturen sind Teil der Biodiversität.

Gefährdung der Biodiversität

"Zwei globale Umweltkatastrophen, der Verlust der Biologischen Vielfalt und der Klimawandel, sind die vorherrschenden Probleme dieses Jahrtausends. Biodiversitätsverlust, das Aussterben von Millionen von Arten, ist eine sichtbare Konsequenz der ausufernden Ausbeutung des Planeten durch den Menschen. Der durch Treibhausgasemissionen beeinflusste Klimawandel droht unsere Atmosphäre zu verändern und somit auch die Lebensbedingungen auf der Erdoberfläche für uns Menschen und jede andere Spezies,»

- Thomas E. Lovejoy und Lee Hannah

$\rightarrow$ Ohne Biodiversität gibt es keine Zukunft für die Menschheit.

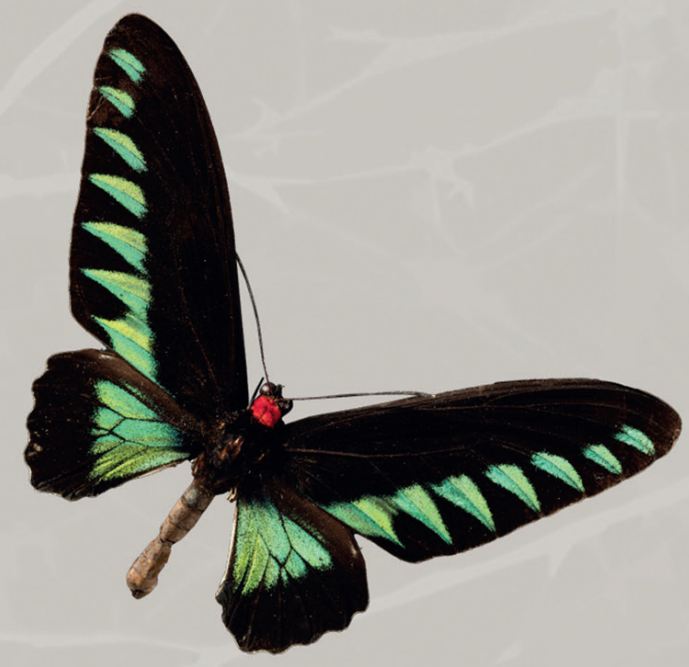




\section{Was ist Biodiversität?}

Warum sollten wir sie bewahren und schützen?

Was ist der Wert von Biodiversität?
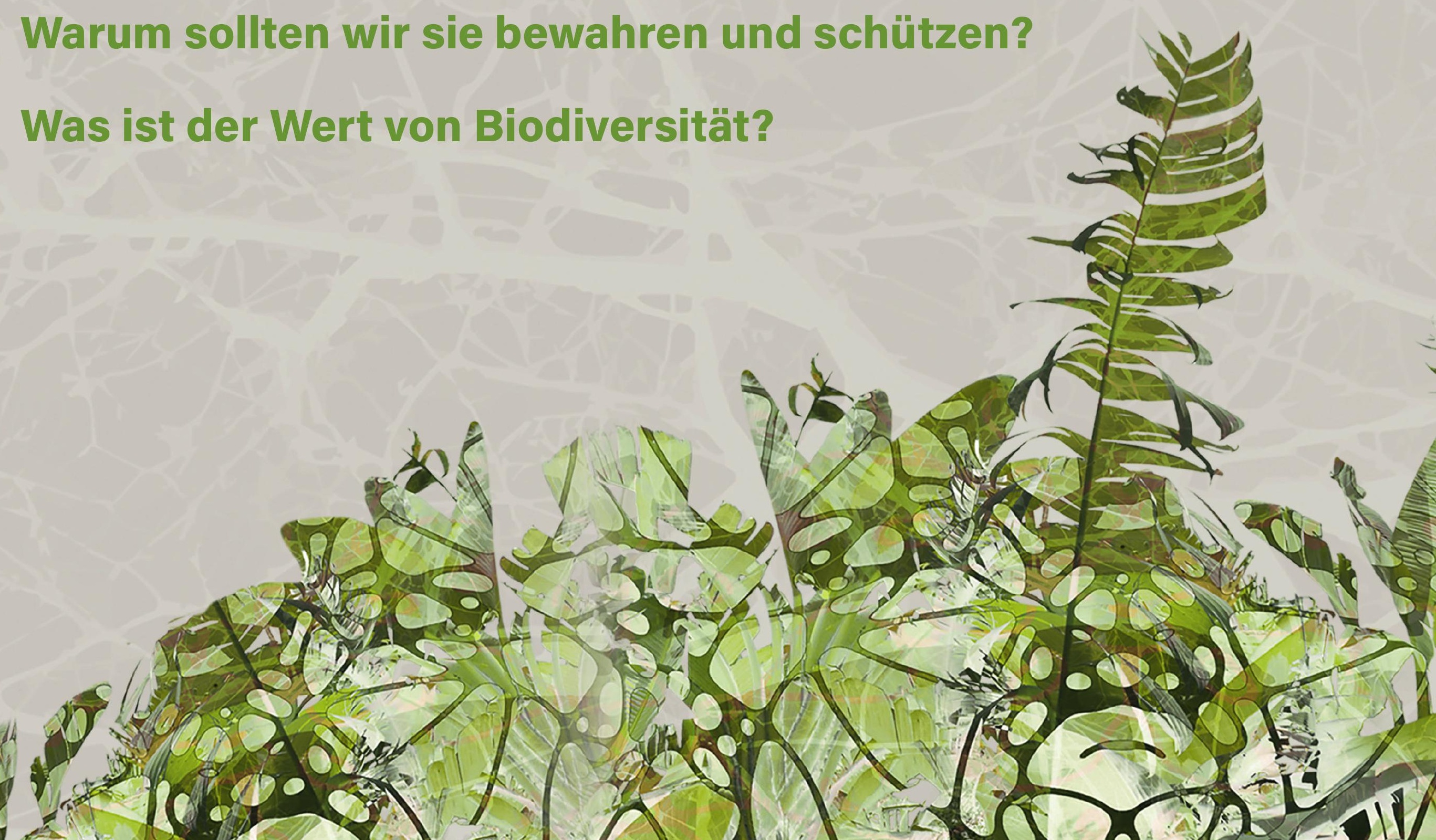


\section{Das Ziel}

«Wir befinden uns an einem kritischen Punkt, was die Biodiversität der Erde angeht, ein unmittelbares Resultat menschlichen Einflusses. Um diese Herausforderung anzunehmen, ist es wichtig, mehr über die Mechanismen, die die biologische Vielfalt hervorbringen und erhalten, zu wissen und die Vorhersage, wie Ökosysteme auf den vom Menschen verursachten globalen Wandel reagieren, zu verbessern. Das Ziel der Biodiversitätswissenschaft ist es, inklusiv und fachübergreifend zu sein und naturhistorische, entwicklungsbiologische, genetische und ökologische Kenntnisse und Erkenntnisse, sowie Sozialwissenschaften, zu vereinen.»

- Rafael Zardoya
Göttingen, die Stadt, die Wissen schafft, ist eng mit ihrer Universität und mit der Karriere von mehr als 40 Nobelpreisträger*innen verbunden. Derzeit arbeiten auf dem Göttinger Campus Forscher*innen vieler exzellenter Abteilungen im breiten Feld der Biodiversitäts- und Nachhaltigkeitsforschung. Im Biodiversitätsmuseum stellen wir die Forschungsprojekte dieser Gruppen vor, um zu veranschaulichen, was Biodiversität ist, wie sie entstanden ist und welche Wechselwirkungen sie in Ökosystemen hat. Wir, als Menschen, sind ebenso abhängig von der Biodiversität wie wir ein Teil von ihr sind. Aufgrund unseres Einflusses befindet sich die Biodiversität jedoch in einer Krise. Wir werden die erheblichen Auswirkungen menschlicher Aktivitäten auf die biologische Vielfalt des Planeten aufzeigen. Das Verständnis, dass Biodiversität für uns wichtig ist und was dies für unsere Zukunft bedeutet, ist der Schlüssel, um diese Krise zu stoppen.

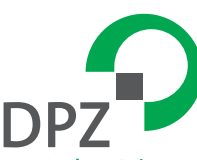

Deutsches Primatenzentrum

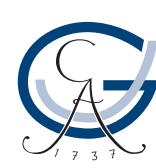

GEORG-AUGUST-UNIVERSITÄT GÖTTINGEN

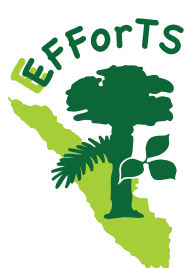




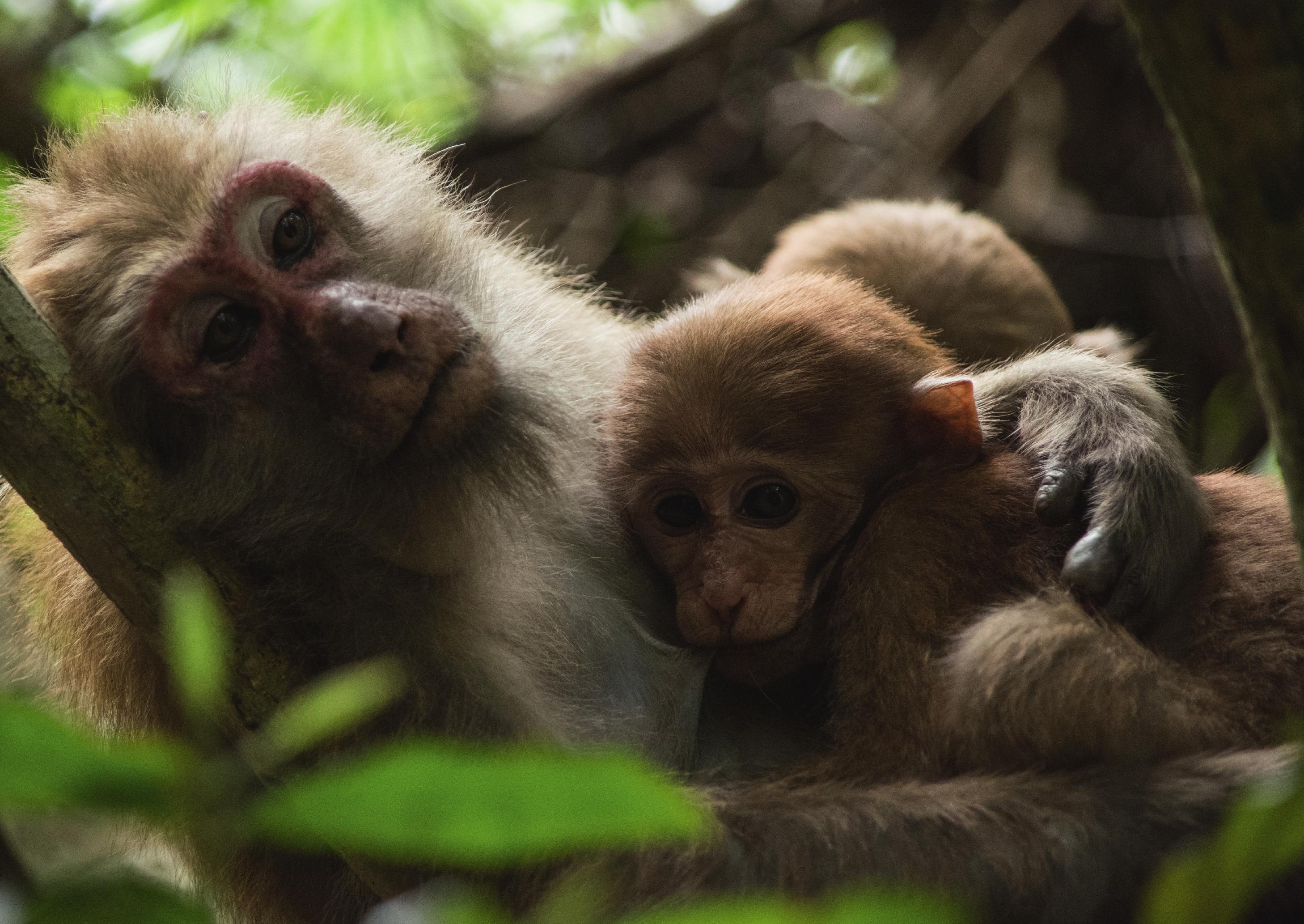




\section{Unser Vorhaben \\ Das Museum}

\section{Aus dem Zoologischen Museum wird das Biodiversitätsmuseum.}

Die zoologische Sammlung der Georg-August-Universität Göttingen hat eine lange Tradition und war ursprünglich Teil des 1773 gegründeten Königlichen Academischen Museums. Im Jahr 1878 zog die zoologische Sammlung zusammen mit der geologischen und mineralogischen Sammlung in das neu erbaute Naturhistorische Museum an der Berliner Straße um.

Im Laufe des letzten Jahrhunderts wurde jedoch die Ausstellungsfläche der zoologischen Sammlung langsam reduziert, während Forschungsabteilungen in das Gebäude einzogen. Inzwischen wurde der Wert von Museen und musealen Sammlungen wiederentdeckt. Das Gebäude an der Berliner Straße wird derzeit saniert und die zoologische Sammlung ist an andere temporäre Standorte in und um Göttingen umgezogen. Im Jahr 2023 soll im zweiten Obergeschoss des ehemaligen Gebäudes des Naturhistorischen Museums das neue Biodiversitätsmuseum eröffnet werden. Das erste Obergeschoss und ein Teil des Erdgeschosses werden das "Forum Wissen" beherbergen, das 2021 eröffnet wird. Diese Storyline legt den Grundstein für eine neue Ausstellung, die sich mit dem Thema Biodiversität beschäftigen wird.

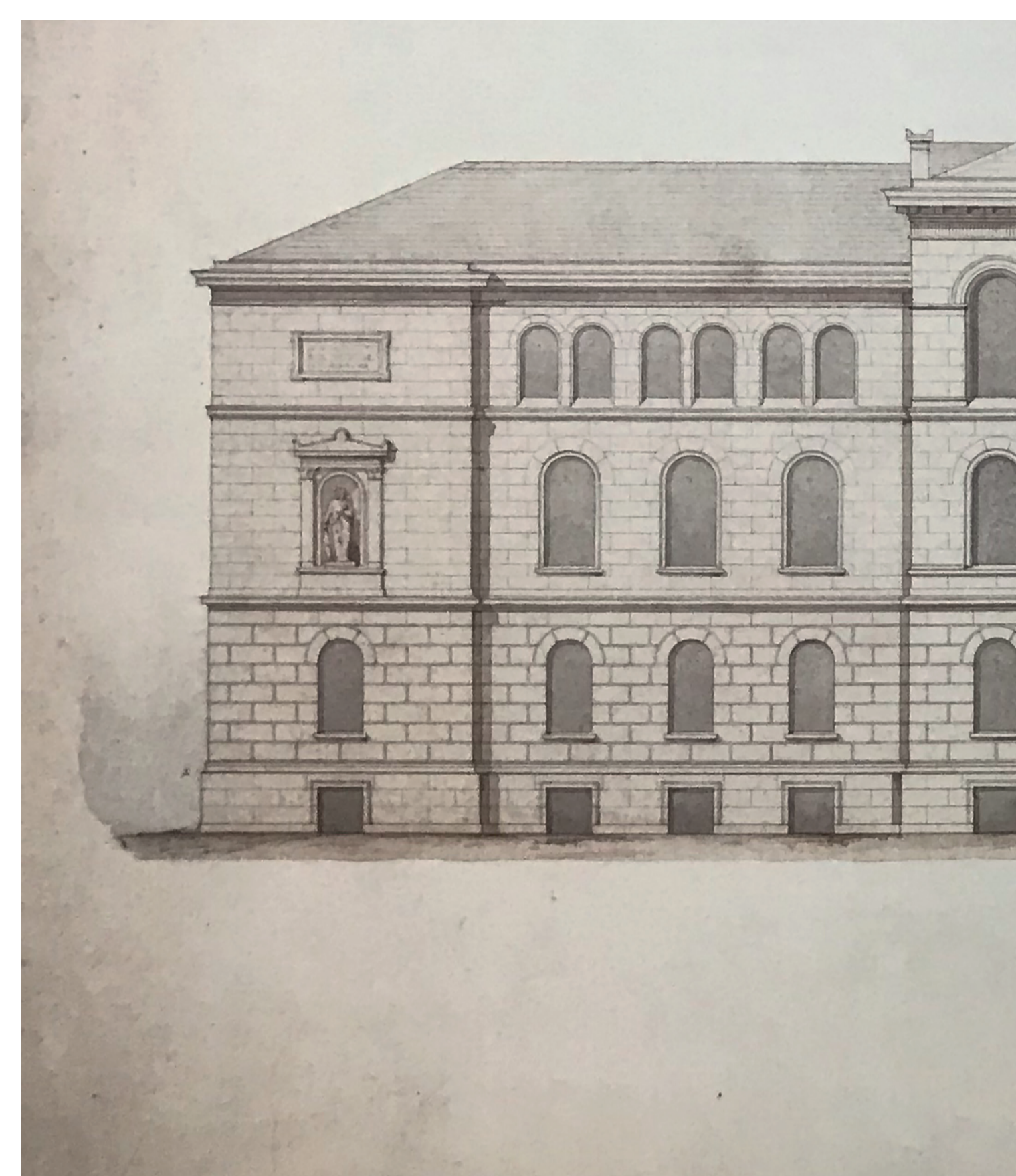

Gebäude des ehemaligen Naturhistorischen Museums in der Berliner Straße 


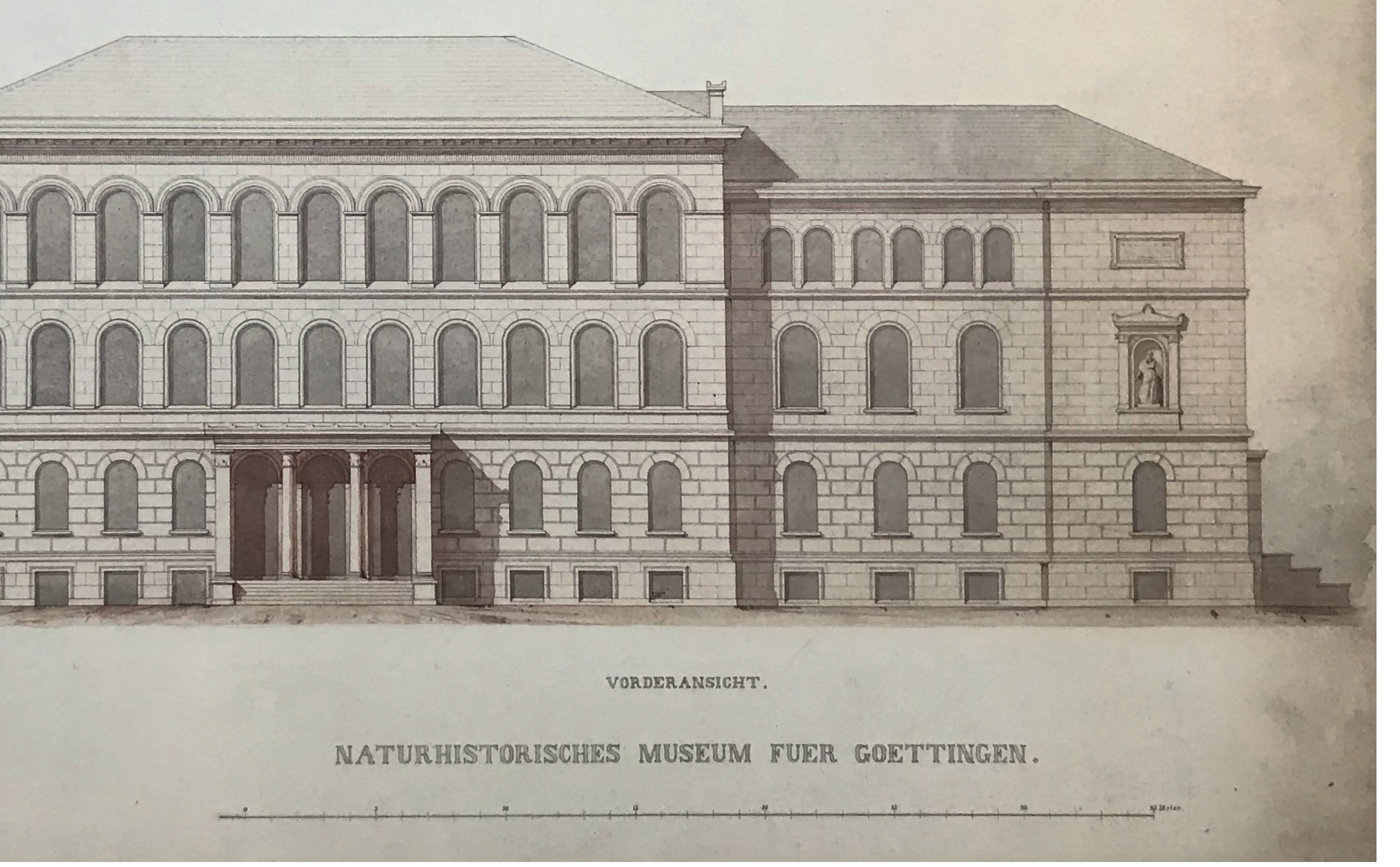




\section{Unsere Intention}

Das übergeordnete Ziel des Museums ist die Förderung und Unterstützung einer wissenschaftlichen Kultur in der Gesellschaft. Unsere Ausstellung soll in den Besucher*innen Begeisterung für die Natur und deren Erforschung wecken und somit auch die wissenschaftliche Arbeit der Universität einer breiten Öffentlichkeit zugänglich machen.

Wissenschaft - das ist die Botschaft - findet nicht in einem Elfenbeinturm statt und die gewonnenen Erkenntnisse haben Relevanz für unser aller Leben. Davon ausgehend wollen wir unseren Besucher*innen Werkzeuge an die Hand geben, damit sie selbst Akteure in ihrem Umfeld werden können. Darüber hinaus stellen wir uns das Museum als Treffpunkt und Bindeglied zwischen diversen Interessensgruppen vor. Als universitäre Einrichtung ist das Museum natürlich durch eine starke Verbindung zur wissenschaftlichen Gemeinschaft gekennzeichnet. Es soll daher auch als Treffpunkt für verschiedene wissenschaftliche Gruppen dienen und dabei auch Expertise der Wissenschaftspopularisierung anbieten.
Selbstverständlich bleibt dieses Museum auch weiterhin Teil der Museumsgemeinschaft, beteiligt sich an Plattformen und Netzwerken und wird eng mit dem im selben Gebäude beheimateten „Forum Wissen“ zusammenarbeiten. Ein weiteres Ziel ist es, starke Verbindungen zu den Göttinger Bürger*innen aufzubauen. Es soll ein Raum für Kunst, Denken und Erholung geschaffen werden, der die Kultur der Wissenschaft in der Stadt fördert. Ergänzend zur Ausstellung wird sich das Museum auch direkt an Schulen, Studierende und auch andere Besucher*innen wenden. Über die Zielgruppe der „üblichen Verdächtigen“ unter den Museumsbesucher*innen hinaus werden wir Programme entwickeln, die Minderheiten oder Menschen mit Behinderungen ansprechen.

Die Ausstellung selbst hat diverse didaktische Ziele: Sie wird ein Fenster in die aktuelle Forschung öffnen, aber auch eine intellektuelle und emotionale Reise durch die Wunder der Natur. Sie soll Neugierde, Kreativität und - im Idealfall - Engagement wecken. 


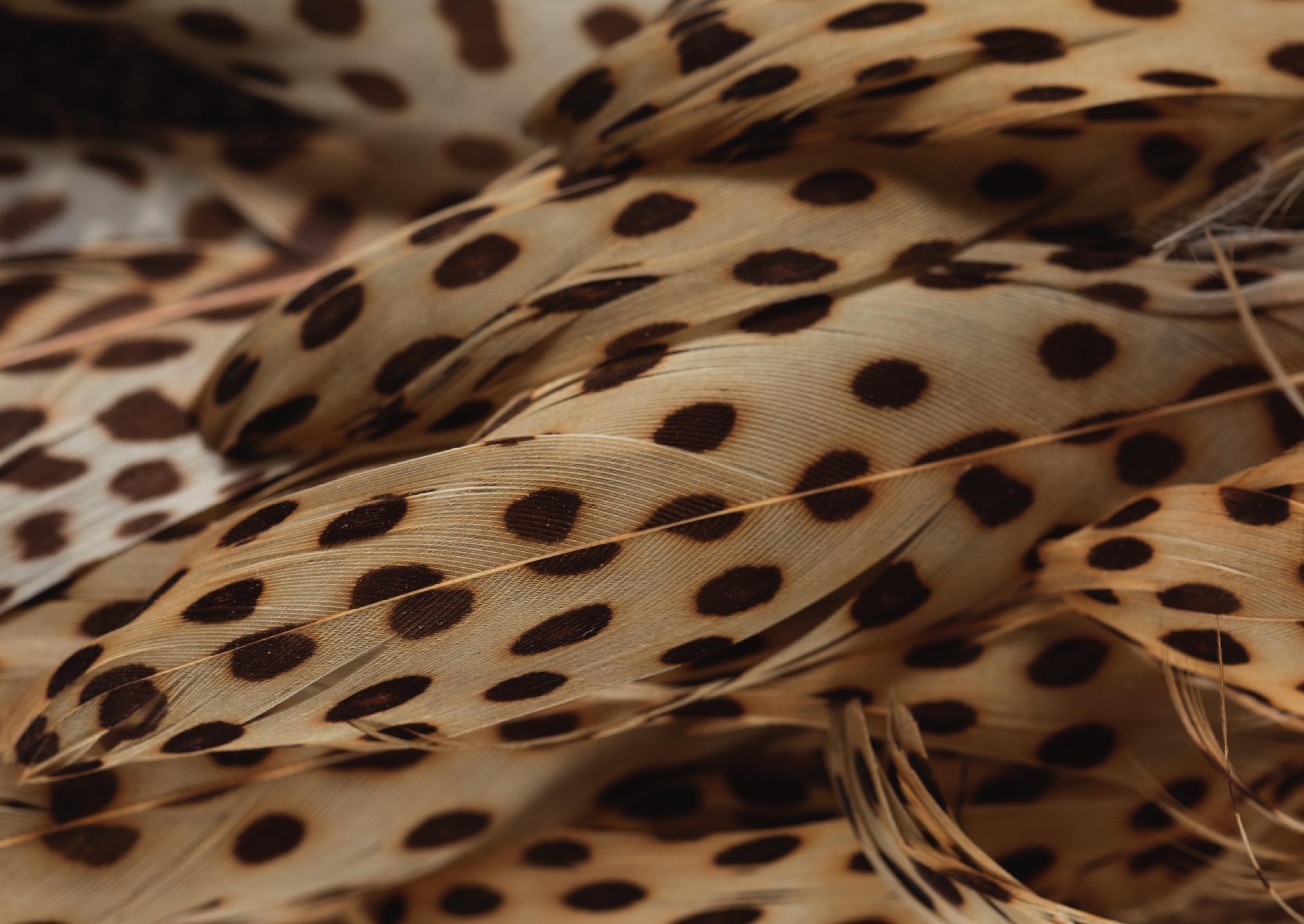




\section{Die Themen}

Grundlagen der Biodiversitätsforschung und Handlungsmöglichkeiten

Die Ausstellung hat drei übergeordnete Themen: Biodiversität und Evolution, Biodiversität und Ökosysteme sowie Biodiversität und der Einfluss des Menschen.

Die Inhalte werden den Besucher*innen in 6 Kapiteln, eingerahmt von einem Prolog und einem Epilog, vermittelt. Biodiversität und Evolution ist der Schwerpunkt in Kapitel 2 und 3, Kapitel 1 und 4 konzentrieren sich auf Ökosysteme, während Kapitel 5 und 6 dem menschlichen Einfluss auf die Biodiversität gewidmet sind. Wie im wirklichen Leben sind die verschiedenen Themen eng miteinander verwoben, weshalb der Inhalt nicht starr in drei Blöcken dargestellt werden kann. Stattdessen begeben sich die Besucher*innen auf eine Expedition und finden auf dem Weg Rätsel, die gelöst wurden, wie auch solche, die noch ungelöst sind. Dabei treffen sie auch auf die professionellen Entdecker, wie z. B. Wissenschaftler*innen, deren Forschung vorgestellt wird.

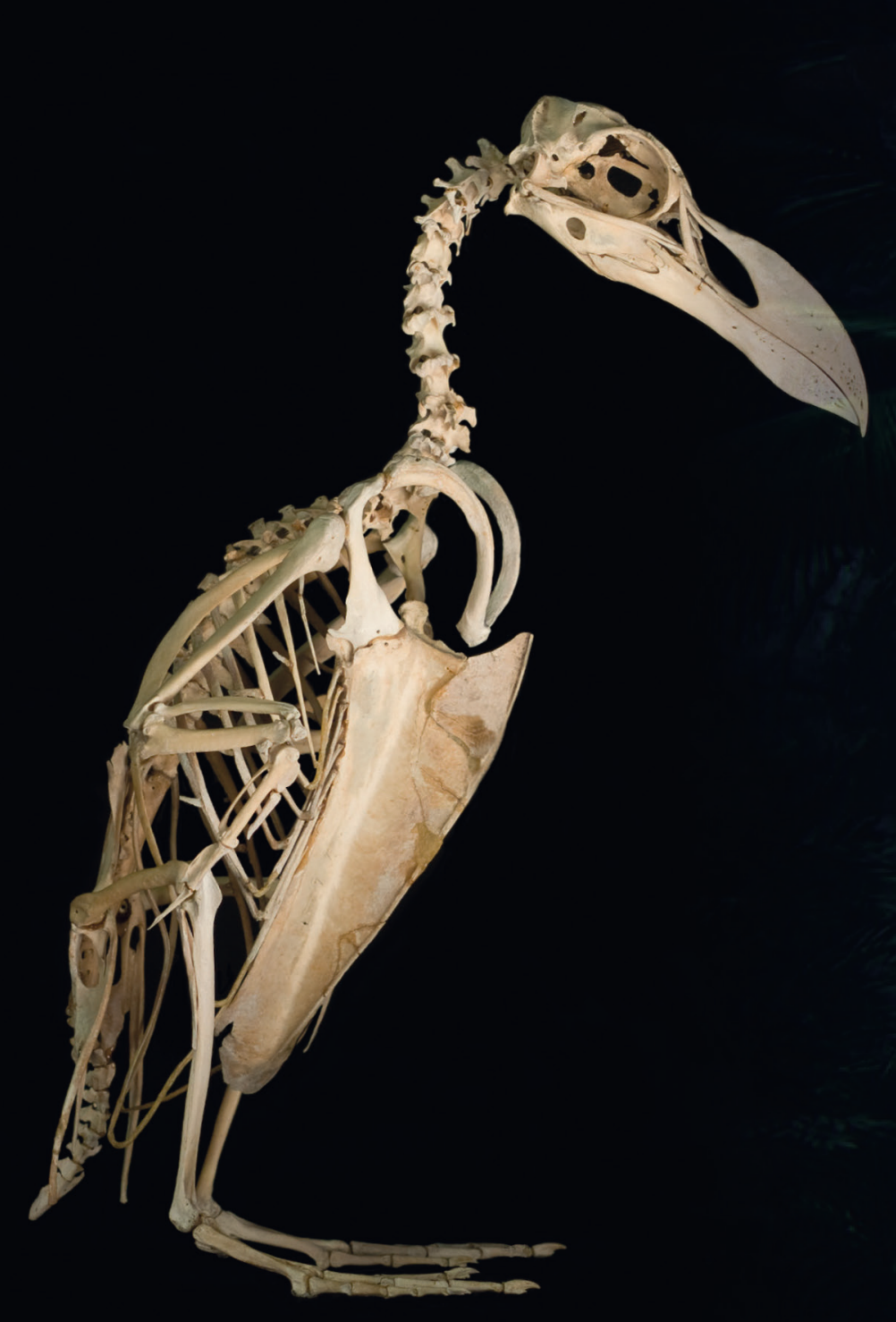




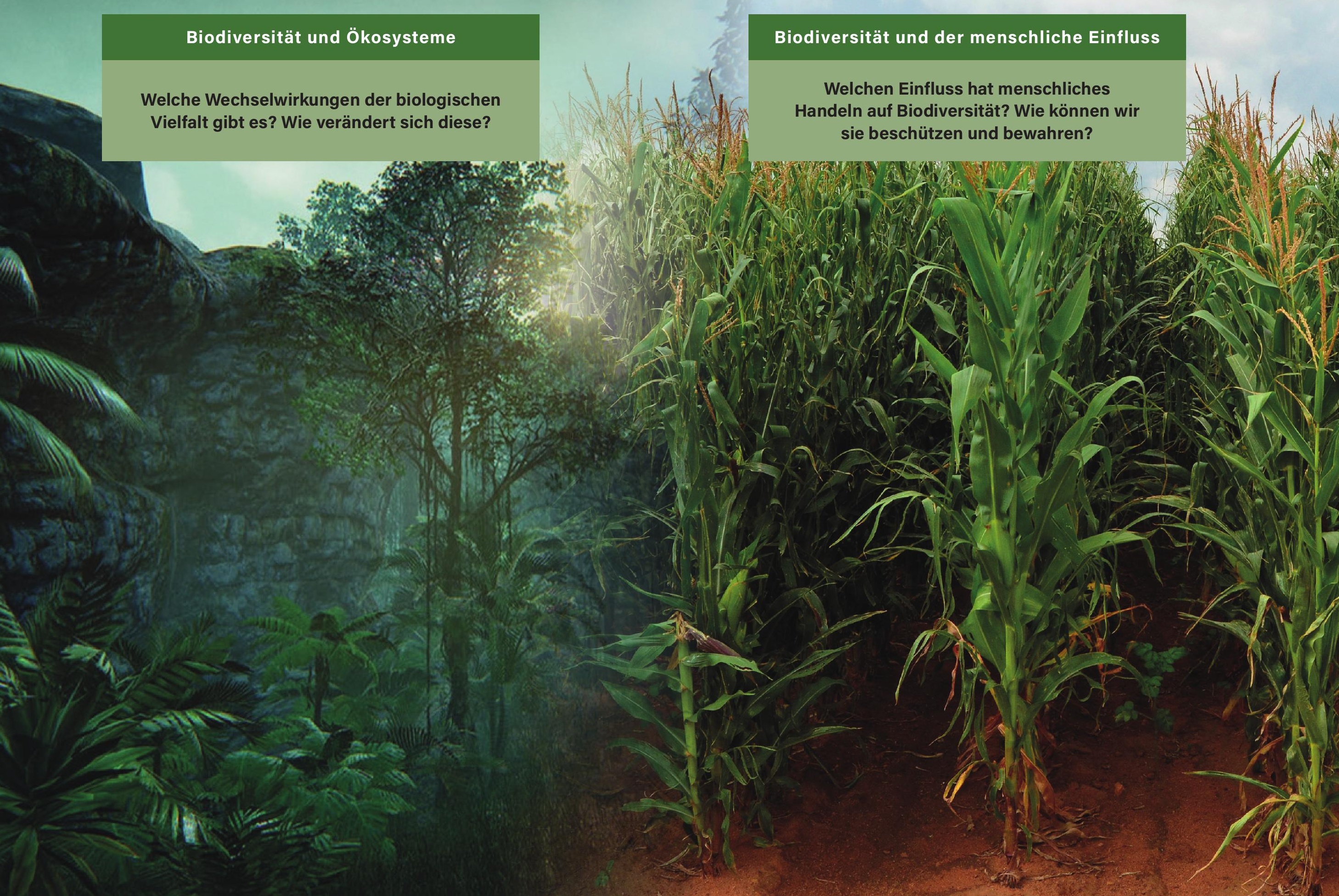




\section{Die Zielgruppen}

\section{Besucher sind Protagonisten}

Das Biodiversitätsmuseum möchte Bürger*innen mit wissenschaftlichem Wissen ausstatten und sie befähigen, aktive Mitglieder in ihrem gesellschaftlichen Umfeld zu werden.

Unsere potenziellen Besucher*innen sind verschiedene Typen von Menschen, in einem breiten Altersspektrum, motiviert durch unterschiedliche Erwartungen. Einige von ihnen werden nur auf der Suche nach Unterhaltung sein, andere werden zusätzlich eine intellektuelle Herausforderung suchen. Das Museum richtet sich an diejenigen, die neugierig sind, diejenigen, die ihrem Umfeld helfen und es verbessern wollen, Experten, kreative Köpfe oder einfach diejenigen, die sich langweilen oder nicht wissen, was sie an einem regnerischen Tag sonst tun sollen.

Sie alle sind willkommen. Bürger*innen engagieren sich auf vielen verschiedenen Ebenen in ihren Gemeinden. Sie sind der Motor, der die Gesellschaft bewegt, und auch der Hauptbestandteil jeder gesellschaftlichen Bewegung. Allerdings haben Bürger*innen oft nicht das Gefühl, dass sie sich an Wissenschaft beteiligen (können). Wissenschaft wird normalerweise als eine Kombination verschiedener unverbundener Pakete von Fachwissen gesehen, die schwer zu verstehen sind und nur wenig mit unserem täglichen Leben zu tun haben. Dieses Museum bietet die Möglichkeit, diese Lücke zu füllen, die verschiedenen Abschnitte zu verstehen und daran teilzunehmen sowie Verbindungen zu finden. Jedes Kapitel ist als eine neue Erfahrung konzipiert. Von einem Raum in den anderen zu gehen, bietet eine allmähliche Steigerung der miteinander verbundenen Konzepte und Gefühle.

Die Gesamterfahrung zielt darauf ab, so inklusiv wie möglich zu sein und auf unterschiedliche Bedürfnisse und Erwartungen einzugehen. Es muss sorgfältig gestaltet und in verschiedenen Ebenen strukturiert werden, die physisch und intellektuell zugänglich sind. Das reicht von Sitzgelegenheiten für ältere Besucher, Grafikdesign und Möbel, die für Rollstuhlfahrer zugänglich sind, über Kreativstationen, die kleine Kinder beschäftigen, bis hin zu Interaktionen, die von Gruppen von Jugendlichen genutzt werden können. Zum Erlebnis gehören maßgeschneiderte Gruppenaktivitäten wie Rallyes, kreative Aufgaben oder Rollenspiele. Die Informations-, Interaktions- und Erlebnismöglichkeiten sind, ebenfalls unter Berücksichtigung der unterschiedlichen Interessenslagen, auf verschiedenen inhaltlichen und physischen Niveaus organisiert, „höher" für Erwachsene und „niedriger" für Kinder. 


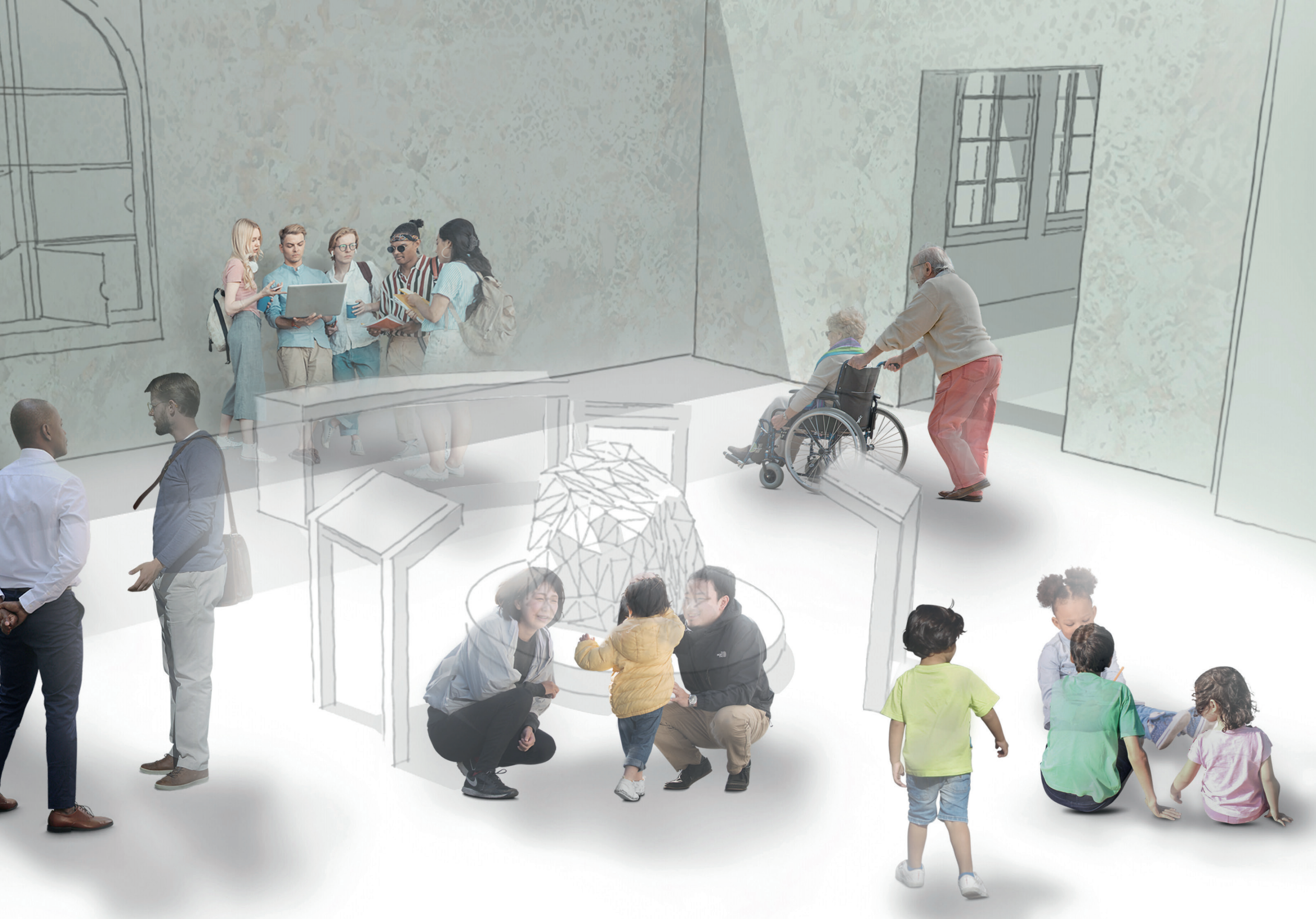




\section{Unser Vorhaben g}

\section{Die Sammlungen}

\section{Wissen und Wunder}

Die Georg-August-Universität beherbergt eine Reihe von botanischen und zoologischen Sammlungen, die ein breites Themenspektrum abdecken.

Allein die zoologischen Sammlungen umfassen mehr als 100.000 Objekte, die von Plattwürmern über ausgestorbene Vögel bis hin zu einem kompletten Pottwalskelett reichen. Viele dieser Exemplare haben neben dem wissenschaftlichen auch einen kulturhistorischen Wert. Das neue Museum wird die Sammlungen im Kontext der Biodiversitätsforschung präsentieren. In jedem Kapitel werden einige sorgfältig ausgewählte Schlüsselexponate aus den Universitätssammlungen ausgestellt, die die Geschichte der Ausstellung illustrieren und stützen. Die Darstellung der Exponate wird je nach Kapitel der Ausstellung unterschiedliche Aspekte betonen. Mal wird die Vielfalt und Fülle der Natur hervorgehoben, mal ein bestimmtes Detail wie ein einzelner Knochen, der die Verwandtschaft zwischen zwei Arten nahelegt.
Sammlung von Algenkulturen (SAG) der Universität Göttingen

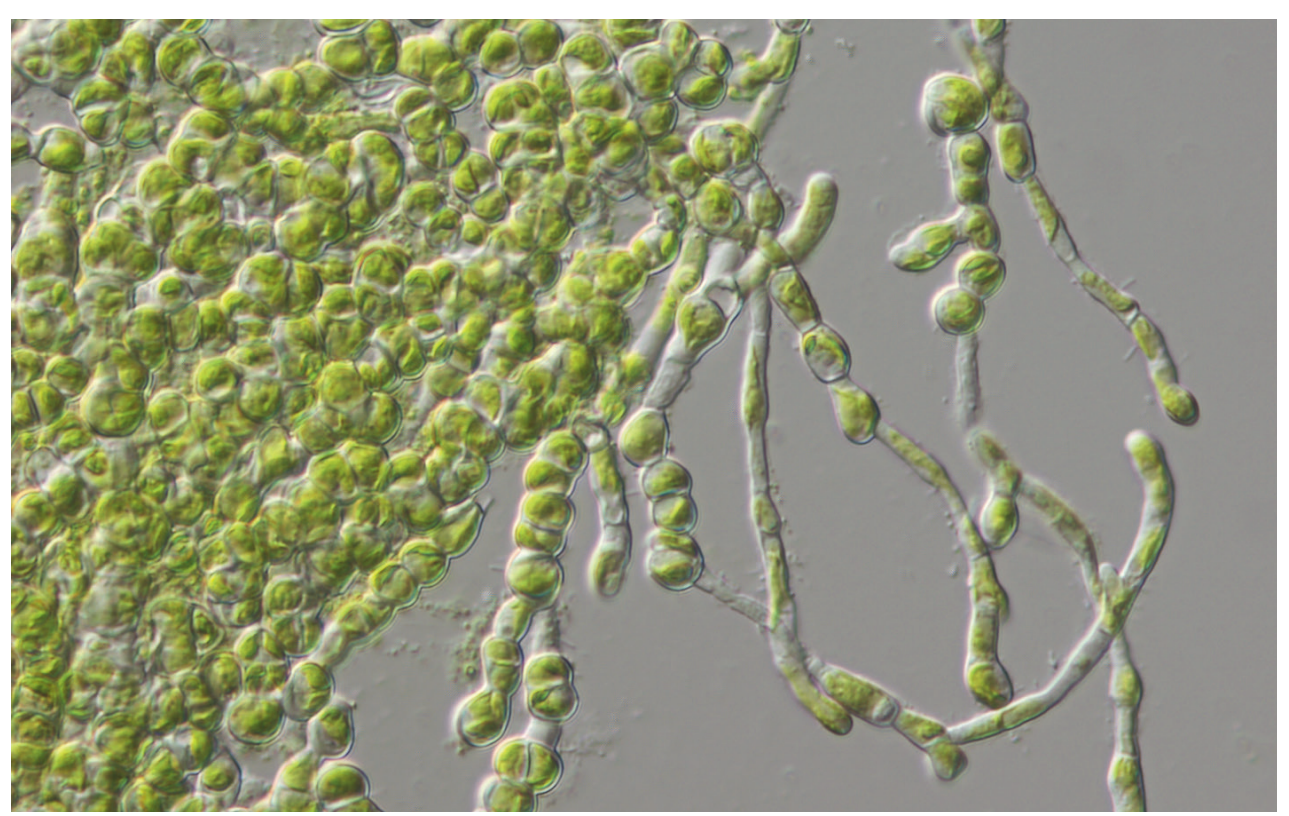

Halofilum ramosum Darienko \& Pröschold, 2017

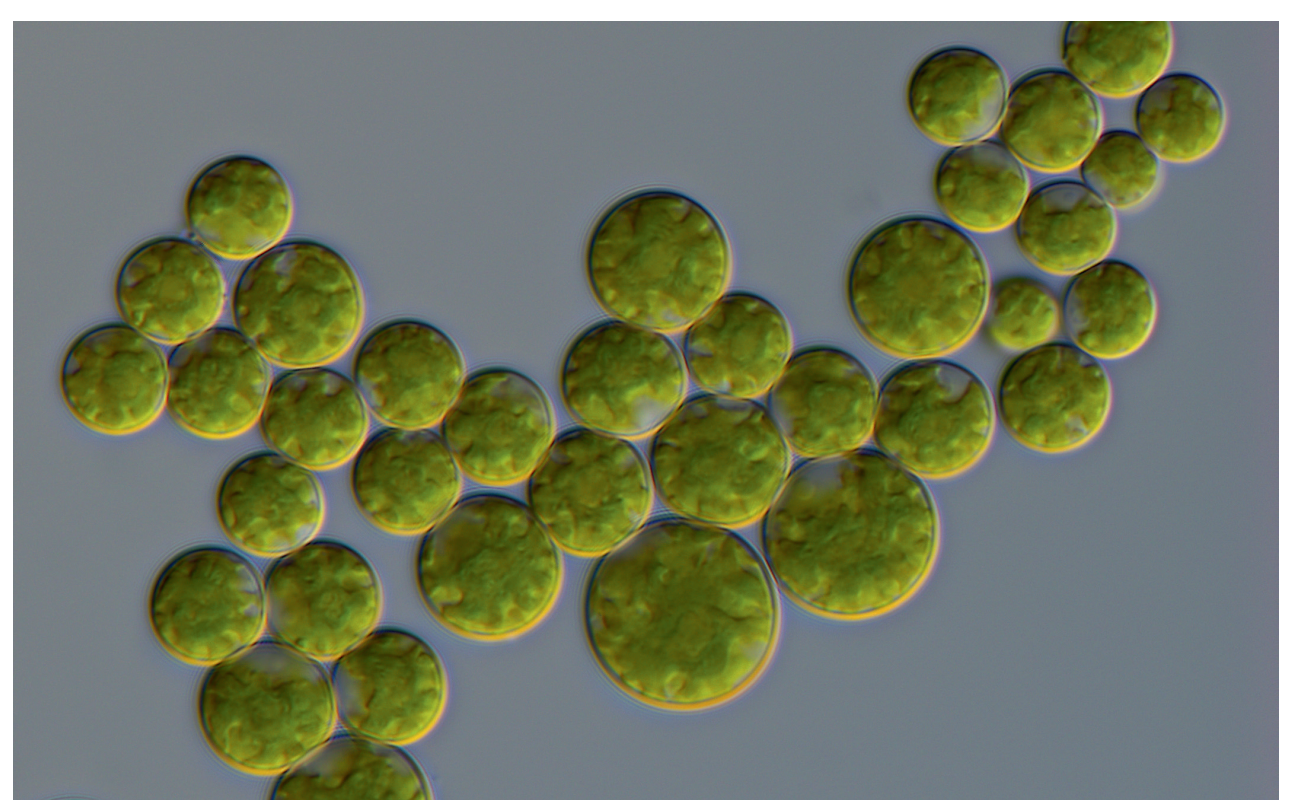

Asterochloris gaertneri Skaloud \& Peksa, 2015 (Fotos von T. Darienko) 


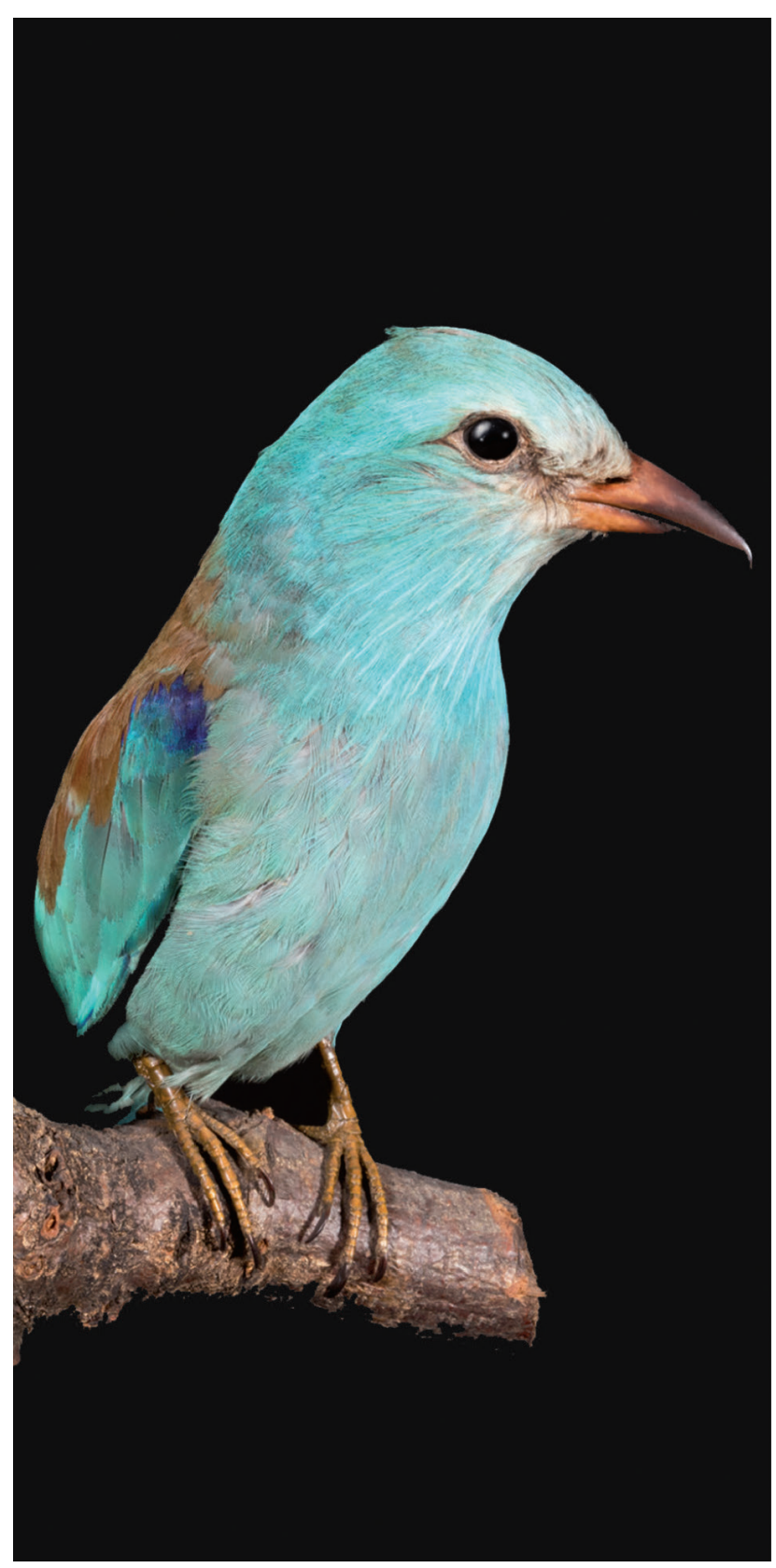

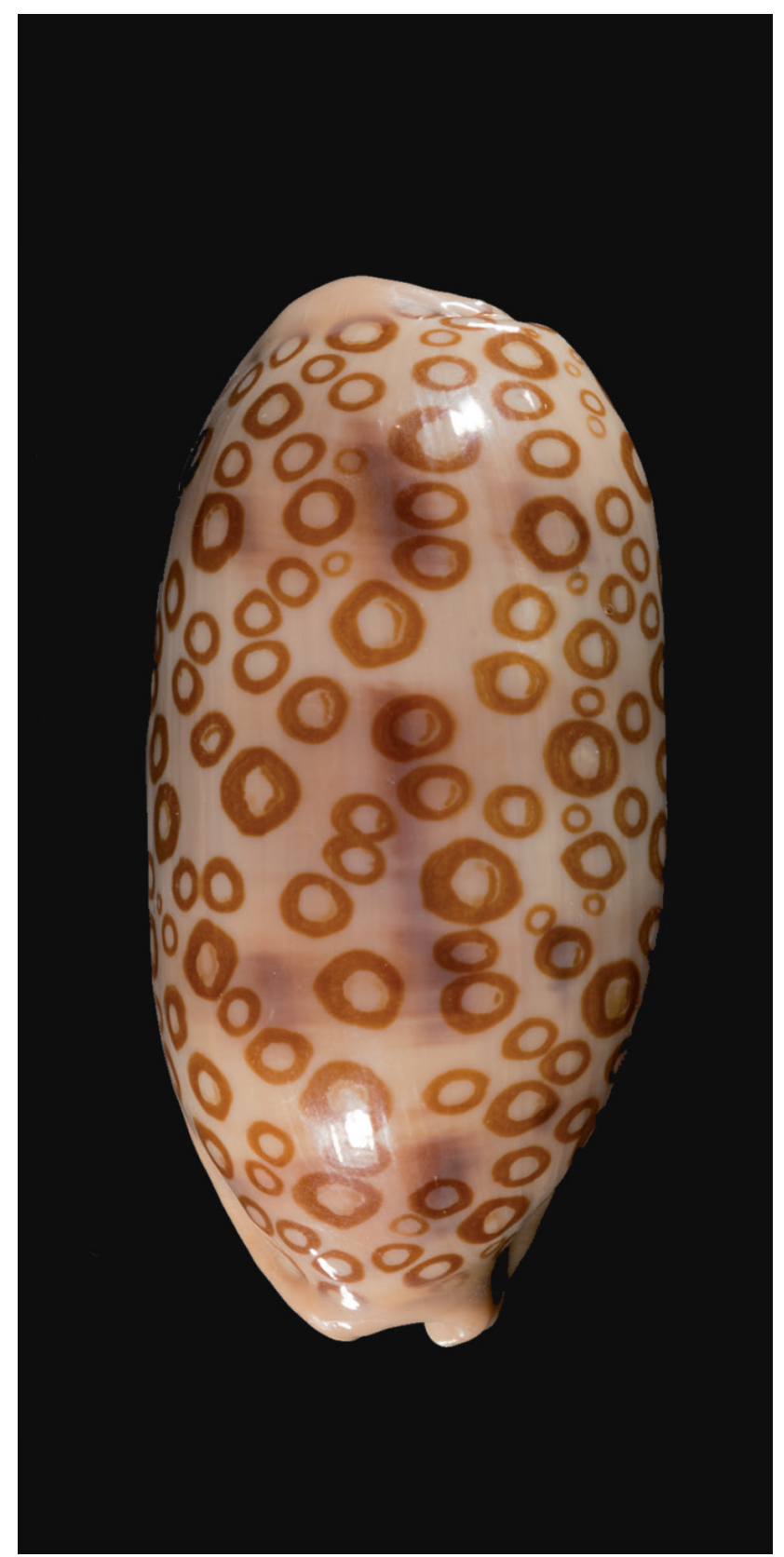

Arestorides argus (Linnaeus, 1758)

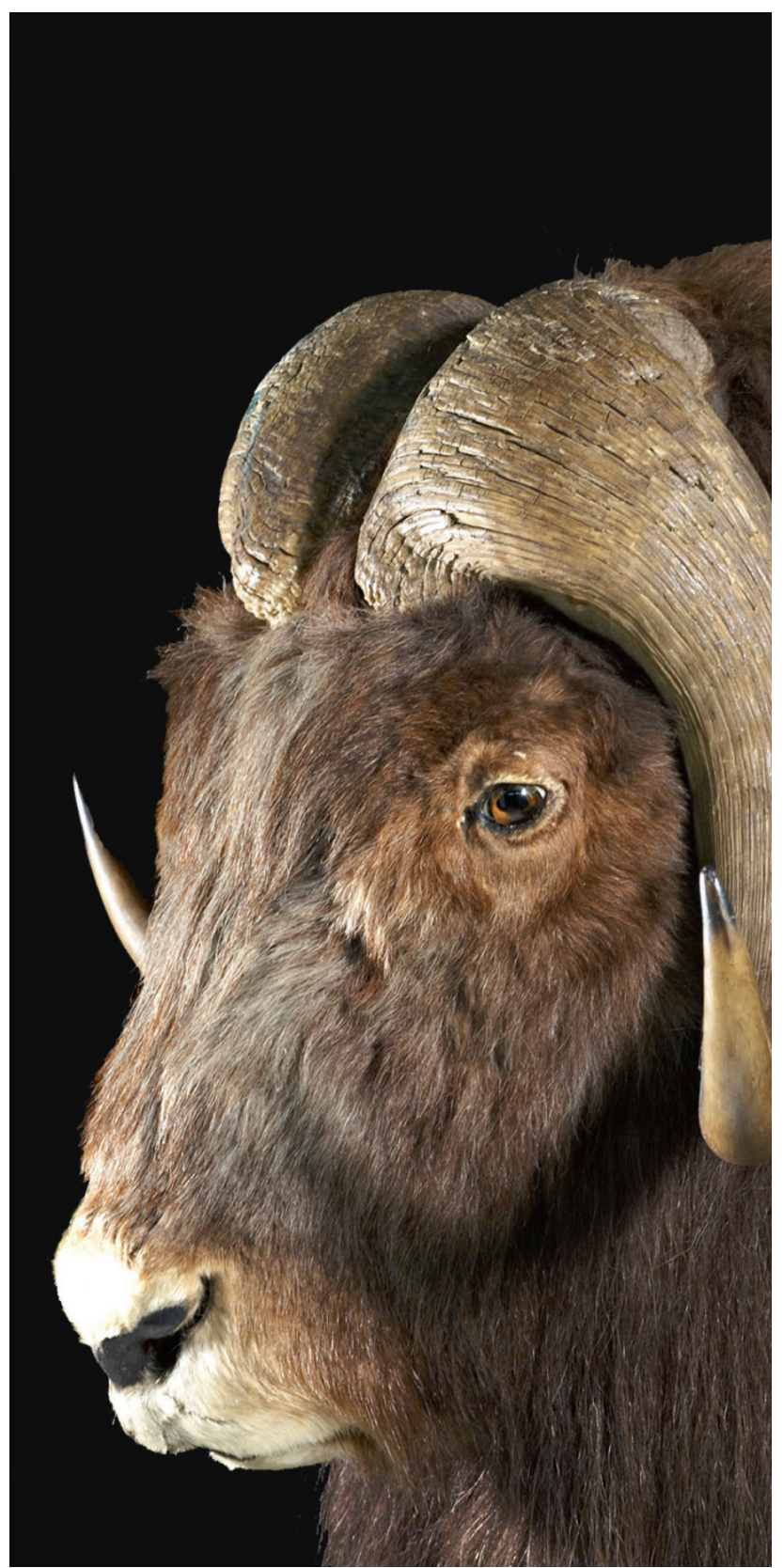

Ovibos moschatus (Zimmermann, 1780) (Foto von C. Fischer) 


\section{Kommunikation \& Didaktik}

Alle Sinne ins Spiel bringen

Neben den Exponaten wird die Ausstellung auch andere Wege zur Kommunikation mit den Besucher*innen nutzen: gedruckte Texte, multimediale Installationen, Mitmachaktionen, Analogien, Simulationen und Stationen für Kreativität und Partizipation. Unterschiedliche Lernkompetenzen werden gefördert.

\section{Denken - konzeptionelle Fähigkeiten: Minds on!}

Die Ausstellung wird einiges erklären müssen, und das kann gleichzeitig lehrreich und unterhaltsam geschehen: Es wird Texte, Infografiken und Videos geben, aber auch echte Präparate und Analogien für bestimmte Vorgänge in der Natur.

\section{Machen - prozedurale Fähigkeiten: Hands on!}

Hands-ons sind eine großartige Möglichkeit, Informationen zu vermitteln. Diese können einfach sein, wie Puzzles, ein Quiz oder Riechstationen, aber auch komplexer wie eine wissenschaftliche Simulation oder ein Rollenspiel mit mehreren Charakteren.
Fühlen - emotionale Erfahrungen: Hearts on!

Eine gute Ausstellung ist eine, die auch die Emotionen der Besucher*innen anspricht. Wir wollen, dass die Besucher*innen über die Vielfalt und Schönheit der Natur staunen. Aber auch der Verlust (in Form des Aussterbens) wird dargestellt sowie dessen Beschleunigung durch menschlichen Einfluss. Und wir werden zeigen, wie Wissenschaftler die Biodiversität erforschen und sich für deren Erhalt einsetzen. Den Besucher*innen werden Citizen-Science-Projekte vorgestellt und Möglichkeiten aufgezeigt, sich für den Naturschutz zu engagieren. Dies soll die Öffentlichkeit motivieren, sich aktiv an Schutz und Erhaltung der Artenvielfalt zu beteiligen.
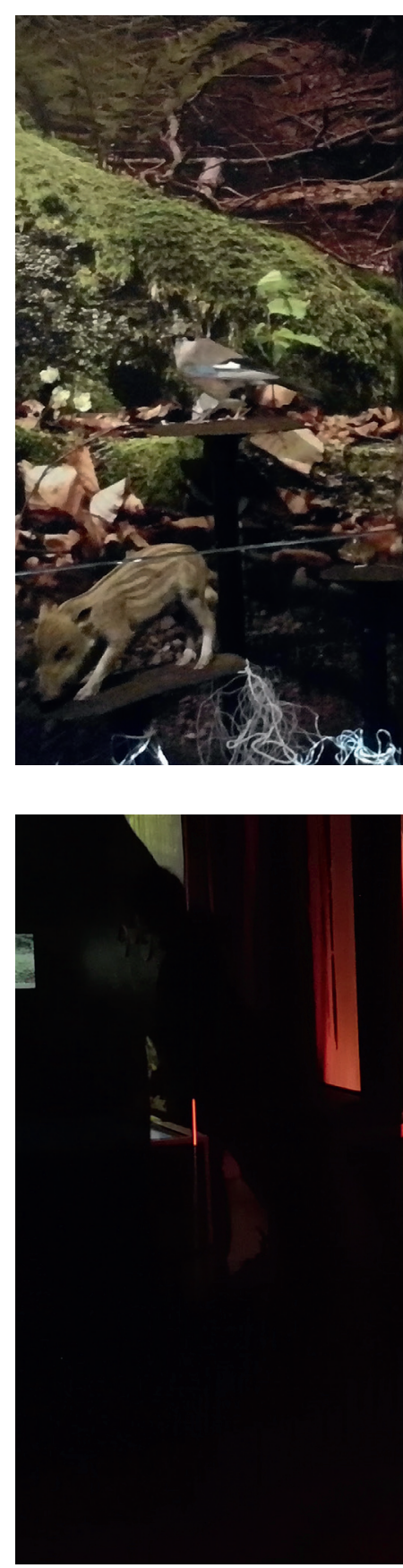


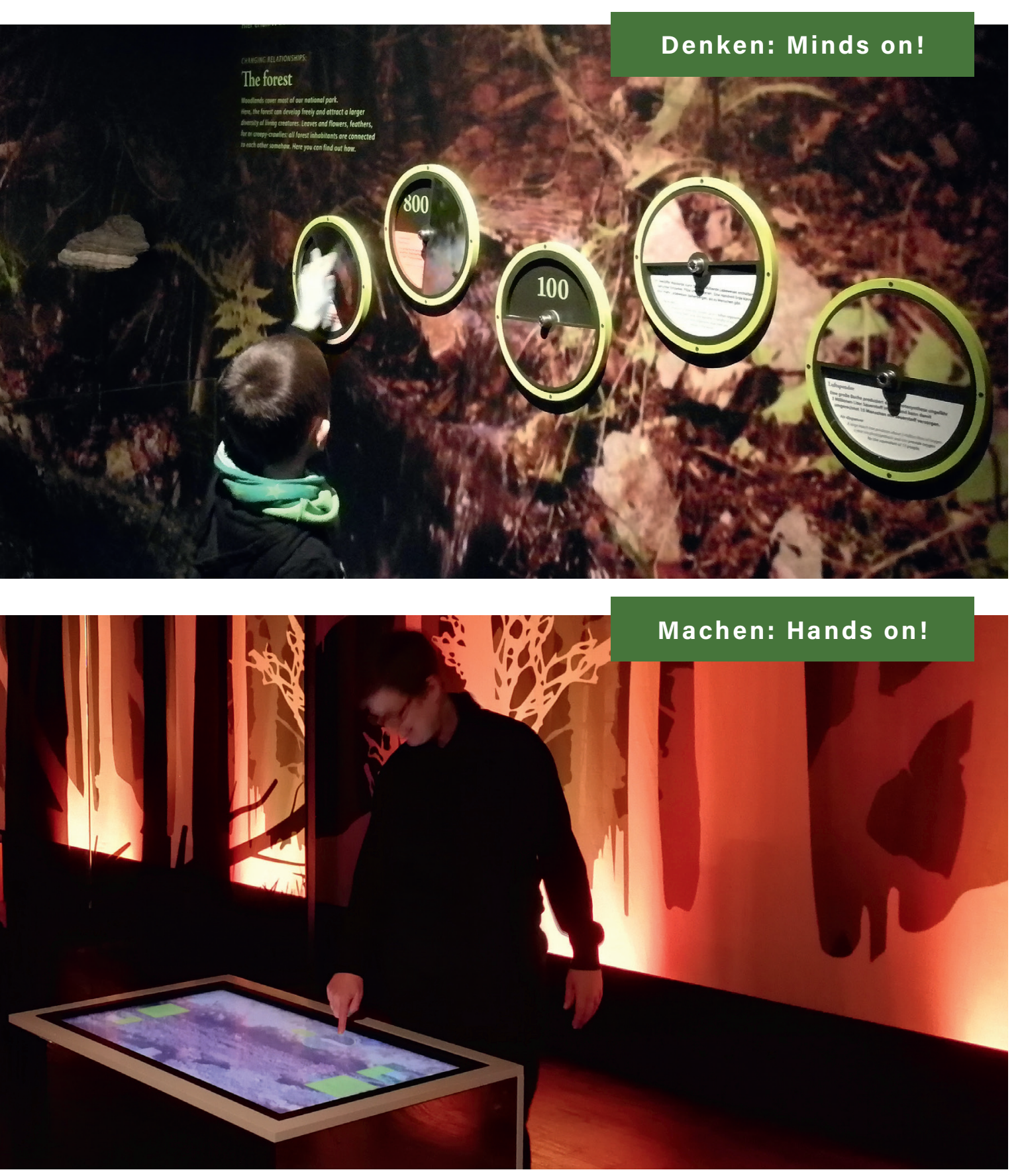

\section{Fühlen: Hearts on!}

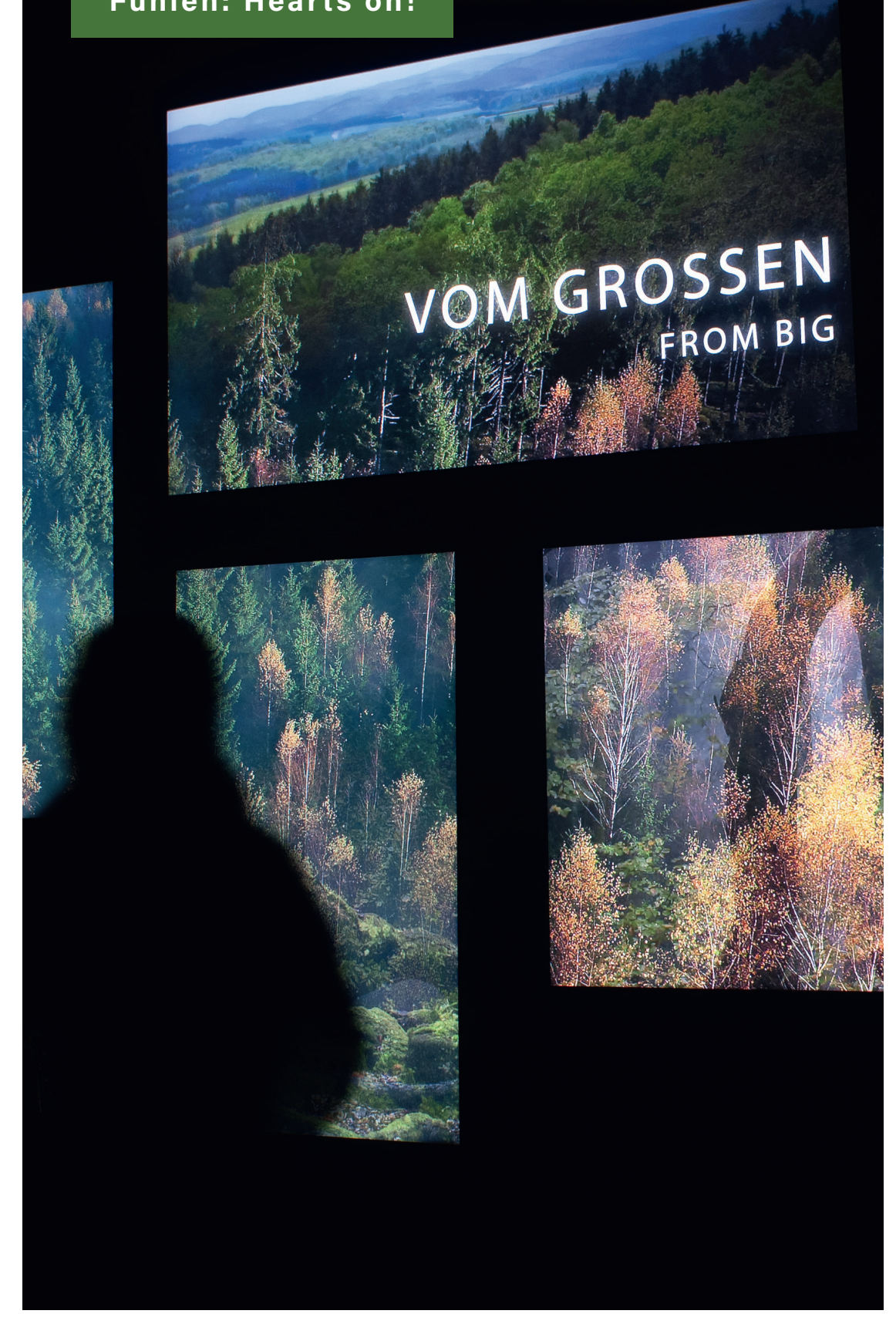



«Wir wurden nicht durch eigenes Verschulden oder durch bewusste Befolgung irgendeines kosmischen Plans, sondern durch die Macht eines glorreichen evolutionären Zufalls, den wir als Intelligenz begreifen, zu den Sachwaltern der Kontinuität des Lebens auf der Erde. Wir haben uns zwar nicht um die Rolle gerissen, aber wir können sie auch nicht ablehnen.»

- Stephen Jay Gould

\section{Das Konzept}




\section{Wissenslücken \& wie man sie füllt}

Biodiversitätswissenschaften sind eine relativ neue Disziplin und nur wenige Menschen abseits der akademischen Welt haben ein vollständiges Verständnis davon, was „Biodiversität" genau ist und wie sie sich von dem Begriff „Artenvielfalt" unterscheidet.

Während Grundlagen der Evolutionstheorie und der Ökologie in der Schule gelehrt werden, haben die meisten Menschen kein umfassendes Wissen über die komplexeren Zusammenhänge. Das macht manchmal die praktische Umsetzung der Erhaltung der Biodiversität schwierig. Dies wird immer wieder deutlich, wenn beispielsweise ein Bauprojekt aufgrund einer seltenen Art gestoppt wird. Häufig von der Öffentlichkeit als „unwichtig" angesehen, bemühen sich Umweltschützer*innen, die Dringlichkeit des Arterhalts zu erklären.
Dieser Mangel an Verständnis ist nicht überraschend einige Mechanismen, die dem Verlust der Artenvielfalt zugrunde liegen, sind nicht intuitiv. Viele Missverständnisse bezüglich der Evolution sind in der Öffentlichkeit immer noch weit verbreitet. So auch die Vorstellung, dass die Evolution eine klare Richtung hat und sich immer vom Einfachen zum Komplexen entwickelt. Daher ist es elementar, in der Ausstellung die Grundlagen der Biodiversitätsforschung unterhaltsam und verständlich zu erklären. Die Besucher*innen können so die nötigen Einsichten gewinnen, um ihre eigene Rolle zu verstehen und ihr Handeln besser einzuschätzen. Die meisten Menschen, vor allem Kinder, lieben die Natur und eine gute Geschichte. Das ist die beste Voraussetzung, um die Schönheit der Natur und die gewundenen Pfade der Evolution zu bestaunen und dabei das Wissen nebenbei aufzusaugen. 


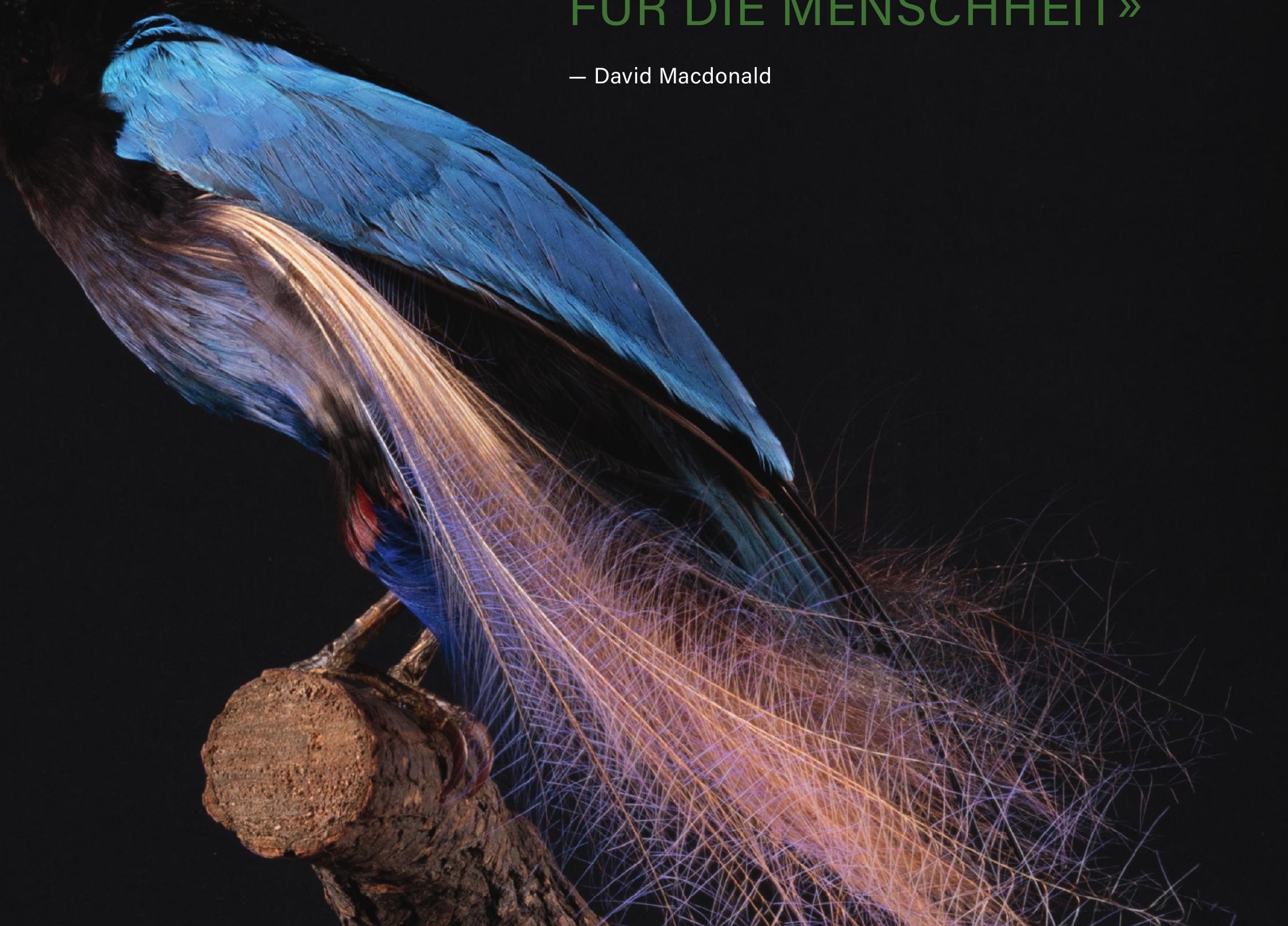




\section{Die Dramaturgie}

Die Ausstellung des Biodiversitätsmuseums wird einen festen Rundgang haben, der die Geschichte in verschiedenen Kapiteln erzählt, beginnend mit grundlegenden Informationen und endend mit komplexen Wechselwirkungen.

Man kann sich die Ausstellung wie ein Pop-up-Buch vorstellen: Jedes Mal, wenn eine Seite umgeblättert wird, entfaltet sich ein neues Wunderwerk und weitere, spannende Informationen werden vermittelt. Jedes Kapitel hat einen visuellen "Konnektor", der den Weg zum nächsten Kapitel weist und sie auf eine überraschende Weise miteinander verbindet. Die Konnektoren werden von den Besucher*innen physisch aktiviert, als Metapher für die Erfahrung: Lernen durch Handeln. 


\section{PROLOG}

Was ist Biodiversität (und warum ist sie so wichtig)?

\section{KAPITEL 1}

Was ist ein Ökosystem?

\section{KAPITEL 2}

Was ist eine Art?

KAPITEL 3

Was ist Evolution?

\section{KAPITEL 4}

Warum ist Biodiversität wichtig?

\section{KAPITEL 5}

Menschlicher Einfluss

KAPITEL 6

Entdecke Biodiversität in deiner Umgebung

\section{EPILOG}

Was kann ich tun?
Die drei Säulen

der Biodiversität

Ein mit Flechten überzogener

Stein in einer kargen Umgebung

Ein Mobile, das die Phylogenie der Insekten darstellt

Der Übergang zwischen

terrestrischem und

marinem Lebensraum

Rekonstruktion eines Dschungels einer indonesischen Insel

Eine Palmölplantage in

Indonesien und eine Mais-

Monokultur in Deutschland

Diorama von

Göttingen

Baum der

Wünsche
Motte

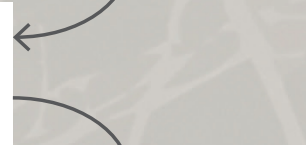

Fledermaus

Seestern

Vogel

Biene 


\section{Räumliche Aufteilung}

Das Biodiversitätsmuseum wird den größten Teil der zweiten Etage des Gebäudes einnehmen, welches ehemals das Naturhistorische Museum in der Berliner Straße beherbergte. Das beeindruckende historische Gebäude neben dem Göttinger Hauptbahnhof ist gut an den öffentlichen Nahverkehr angebunden.
Der Grundriss der räumlichen Aufteilung des zweiten Obergeschosses gab die Inspiration für die Organisation der Ausstellung in einzelne Kapitel. Besucher*innen folgen dem Parcours, wobei jeder Raum eine andere Perspektive auf die biologische Vielfalt repräsentiert und eine andere Szenografie und Atmosphäre hat. Während ein Gebäudeflügel die Ausstellung beherbergen wird, ist der andere Flügel für Labore und interaktive Gruppenveranstaltungen vorgesehen. 
Räume für Gruppenarbeit

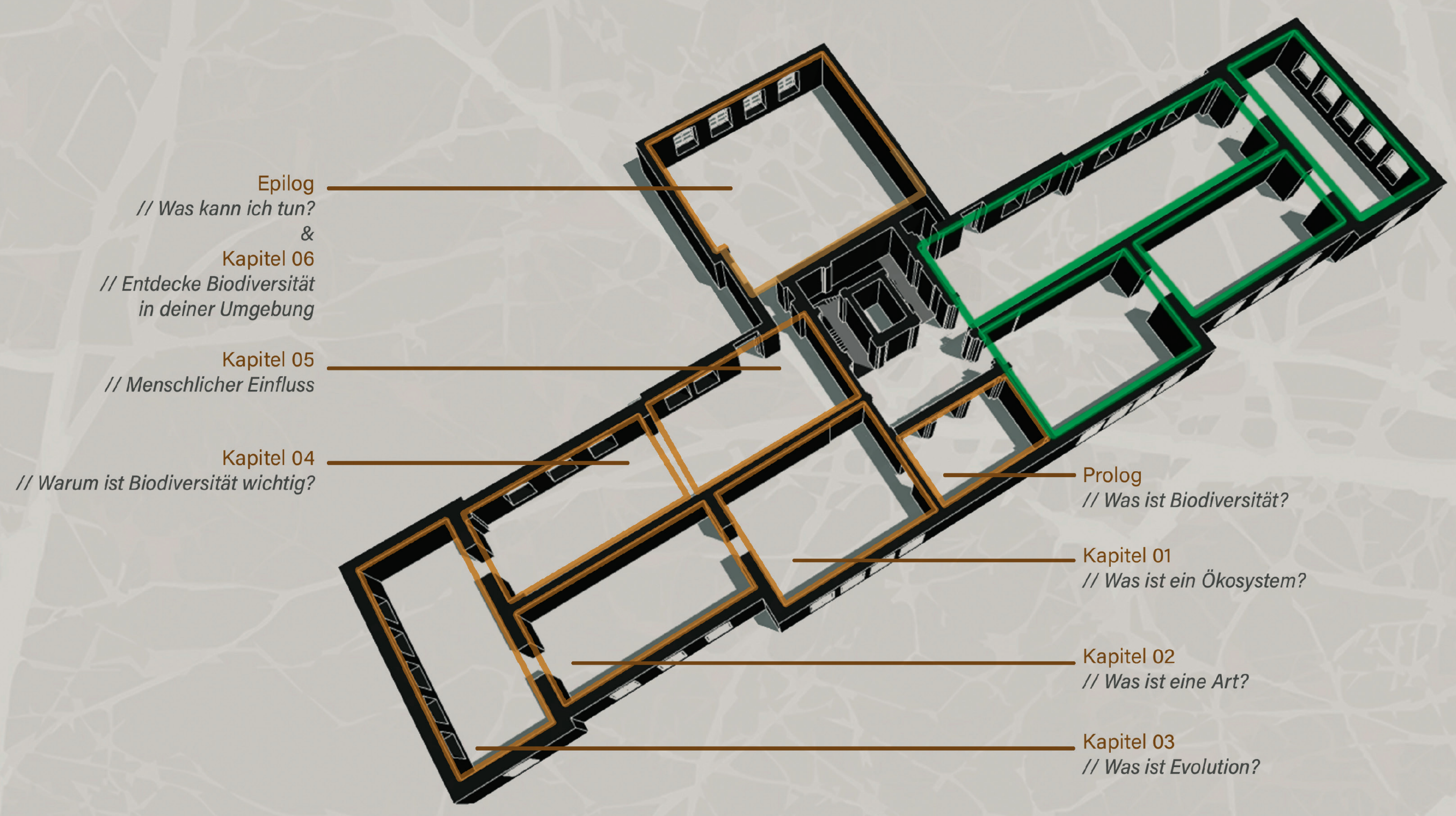




\section{Die Szenografie}

Jedes Kapitel des Museums ist in einem eigenen Raum untergebracht und besitzt seine eigene abgeschlossene Szenografie. Die Besucher*innen können sich von Raum zu Raum bewegen.

Auch wenn jedes Kapitel und damit jeder Raum die gleiche Grundstruktur hat, lassen themenbezogen gestaltete, eindrucksvolle Installationen im Zentrum, umgeben von Interaktionen und "Fensterelementen", jedes Kapitel überraschend anders wirken. Jeder Raum eröffnet eine neue, spannende Welt, in der neue Fragen gestellt und beantwortet werden. Das vermittelt den Besucher*innen das Gefühl, sich auf einer spannenden Entdeckungsreise durch die Wunder der Biodiversität zu befinden.
Der Mittelpunkt des Raumes - der Kristall - ist eine stimmungsvolle und zugleich künstlerische 3D-Darstellung des Kernthemas eines jeden Kapitels. Der Kristall enthält z. B. Nachbildungen eines Ökosystems in Kombination mit Objekten aus den Sammlungen, künstlerische und konzeptionelle Darstellungen des Themas sowie Möglichkeiten zur Interaktion mit seinen Inhalten. Rund um den Kristall sind auch mehrere Medienstationen und Interaktionen in konzentrischen Ringen gruppiert. Die Kapitel werden von den sogenannten „Fensterelementen" eingerahmt: physische Fenster, die den Blick auf die Arbeit von Wissenschaftler*innen eröffnen (Näheres hierzu auf der nächsten Seite).
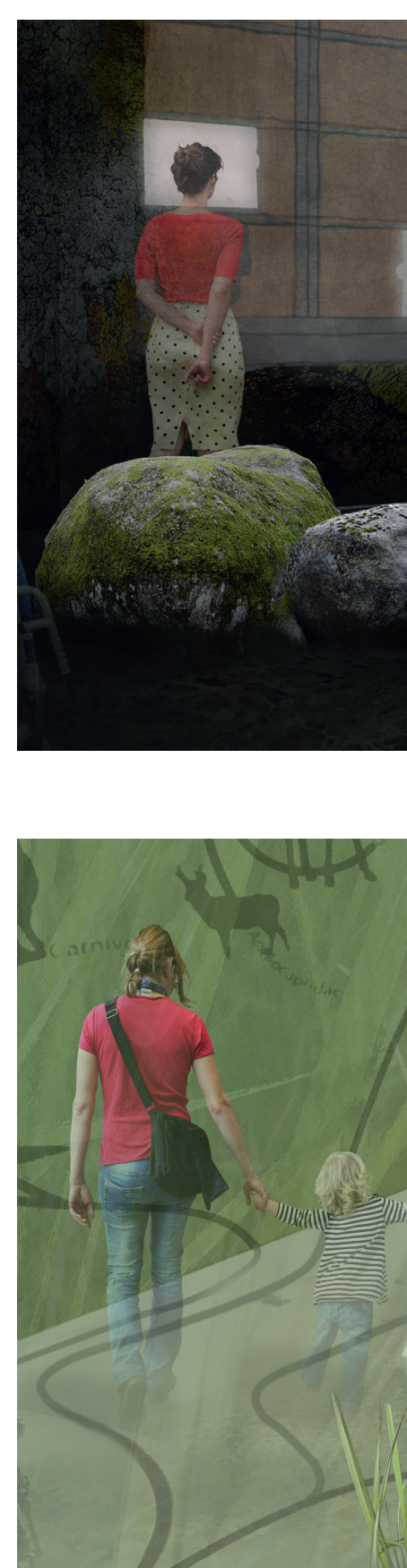


\section{Organisation der Informationen}

\section{Wissensringe und Erlebnisse}

Da die Zielgruppen sehr divers sind, muss sichergestellt werden, dass alle gleichermaßen von Medien \& Informationsebenen, den sogenannten „Wissensringen", angesprochen werden.

Jeder Raum besteht aus mindestens drei verschiedenen Wissensringen in konzentrischer Reihenfolge und verschiedene Informationsstufen umfassend. Der erste Ring beinhaltet die Grundinformationen und befindet sich in der Mitte, der äußerste die Zusatzinformationen.

Der erste, zentrale Ring, als grundlegende Informationsebene, ist als lebendige Szenografie („Kristall") gestaltet, welche durch Überschriften und Zitate ergänzt wird. Sie bestimmt die Stimmung und das Thema des jeweiligen Kapitels. Innerhalb dieser ersten Ebene müssen grundlegende Fragen gestellt und beantwortet werden, beispielsweise ist "Was ist ein Ökosystem?" die zentrale Frage im „Flechtenraum" (Kapitel 1). Die Antwort wird in relativ einfachen Worten am Beispiel der das Gestein überwachsenden Flechte erklärt. In ähnlicher Weise werden so Raum für Raum grundlegende Fragen zu den Hauptthemen innerhalb der entsprechenden ersten Verständnisebene beantwortet. Das ermöglicht den Besucher*innen, auch mit relativ geringer Aufmerksamkeit durch die Aus- stellung zu gehen und trotzdem die Kerninformationen aufzunehmen.

Über dieses Grundverständnis hinaus wird es den Besucher*innen möglich sein, weiterführende Informationen und Erfahrungen zu sammeln. Dies kann in den weiteren "Wissensringen" erreicht werden. So bietet der zweite Ring im „Flechtenraum" beispielsweise vertiefende Informationen über Flechten und Ökosysteme und die Besucher*innen können verschiedene Flechten unter dem Mikroskop betrachten. Auf dieser Ebene werden interaktive Stationen, Tafeln und Objekte als didaktische Hilfsmittel eingesetzt.

Die Wände des Raumes, an denen sich die „Fensterelemente" befinden, umschließen das Kapitel als dritte Ebene. Diese "Fenster" werden wie reale Fenster bedient und können von den Besucher*innen geöffnet werden. Sie ermöglichen den Betrachter*innen den Blick auf Orte außerhalb des Gebäudes und der Ausstellung, wie wissenschaftliche Labore, in denen verschiedene Forscher*innen der Universität Göttingen arbeiten. Wir können sehen, was sie tun, und mit ihnen interagieren. Hierfür werden wir unter anderem audiovisuelle und interaktive Stationen nutzen. 
- - - - - - - - - - - - - - - - - - - - - - - - - - - - - - - - - - - - - - - - - - - - - - - - - - - - - - - - - - - - - - - - - - - - - - -

\section{Kapitel / Raum}

Szenografie, große Headlines und Open-Sound

\section{西}

,

Ring 3

Ein $360^{\circ}$-Blick auf den Kristall ermöglicht dem Besucher

verschiedene Sichtweisen auf das Thema.

Ring 2

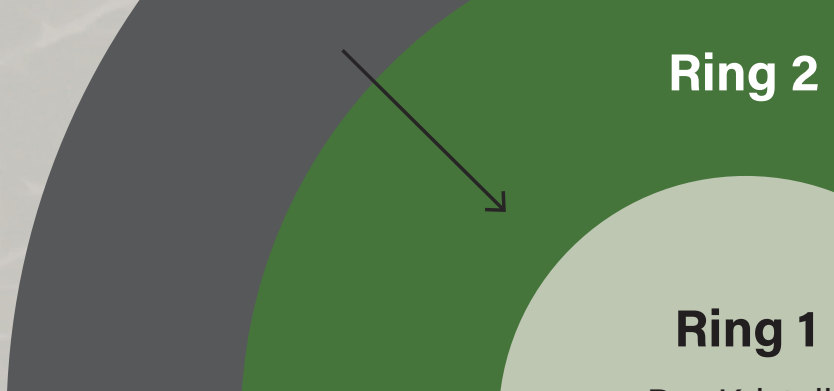

Der Kristall:

zentrales

szenografisches

Element

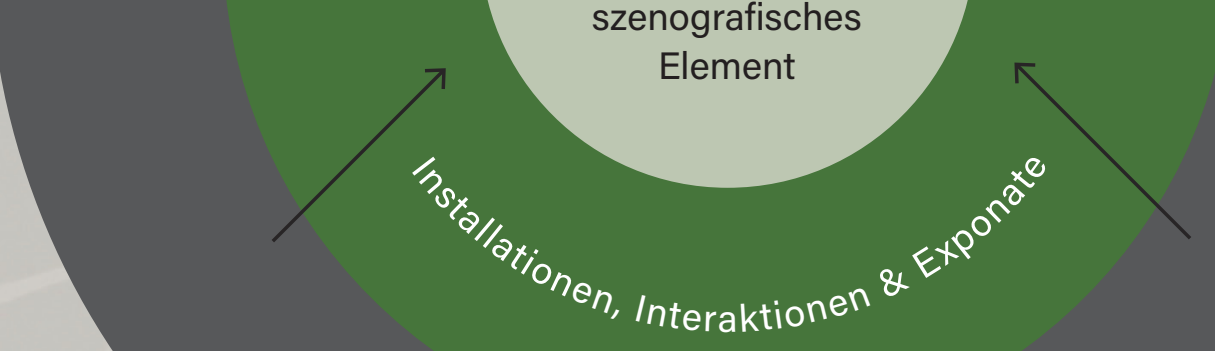




\section{Die Fensterelemente}

Jenseits der Ausstellung

Die „Fenster" sind Elemente in jedem Raum, die einen vertieften (Ein-)blick in ein bestimmtes Thema ermöglichen.

Einige dieser Fenster können von den Besucher*innen geöffnet werden, um "nach draußen", also über die Ausstellung hinaus, zu schauen. Es kann ein Blick in eine andere räumliche Dimension, eine andere Region der Welt oder eine andere Zeitperiode sein. Zudem geben sie auch Einblick in die Wissenschaft und ausgewählte Projekte unserer Wissenschaftler*innen. Die vorgestellten Wissenschaftler*innen sind teilweise Mitglieder des wissenschaftlichen Beirats (Seite 40) und/oder Pionier*innen der Biodiversitätsforschung, unter denen sich auch viele Frauen befinden.

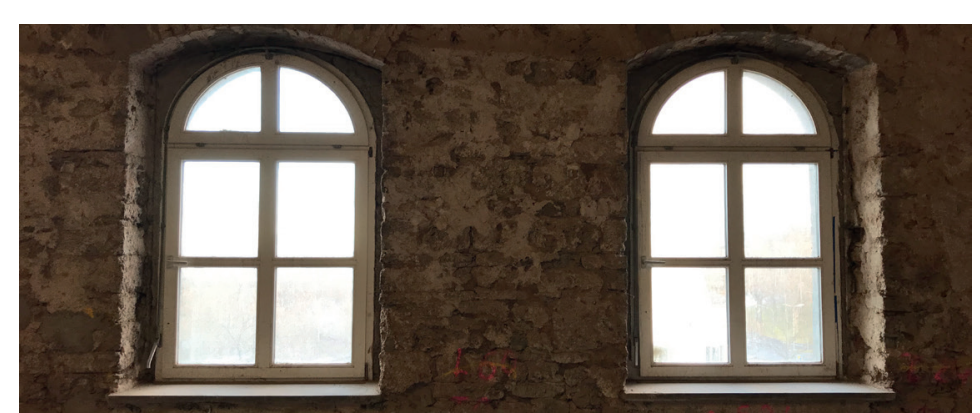

Beispiel 1:

Ein Fenster zu einem Labor, in dem ein wissenschaftliches Projekt erklärt wird. 


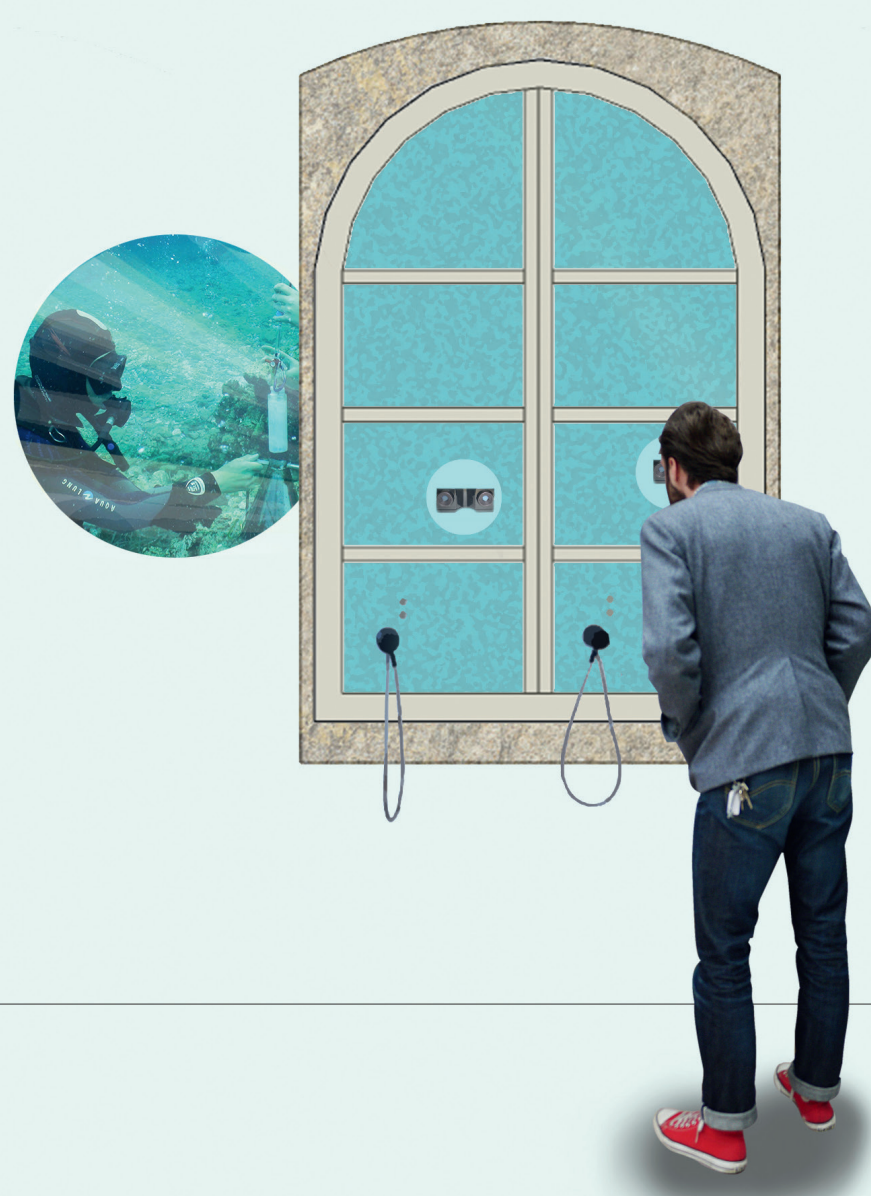

Beispiel 2:

Ein Fenster zum Ozean, welches die Arbeit von Meeresbiologen beleuchtet.

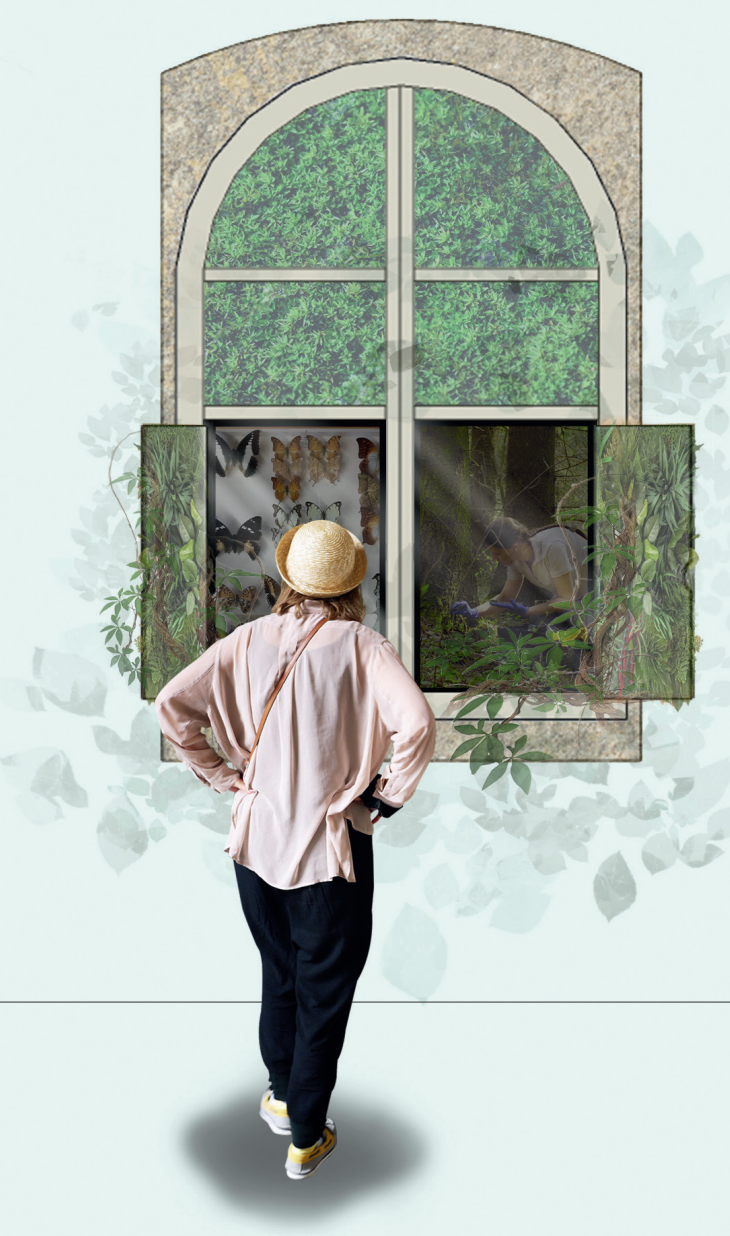

Beispiel 3:

Ein Fenster in den Dschungel, das zeigt, wie die Feldarbeit von Biologen aussieht.

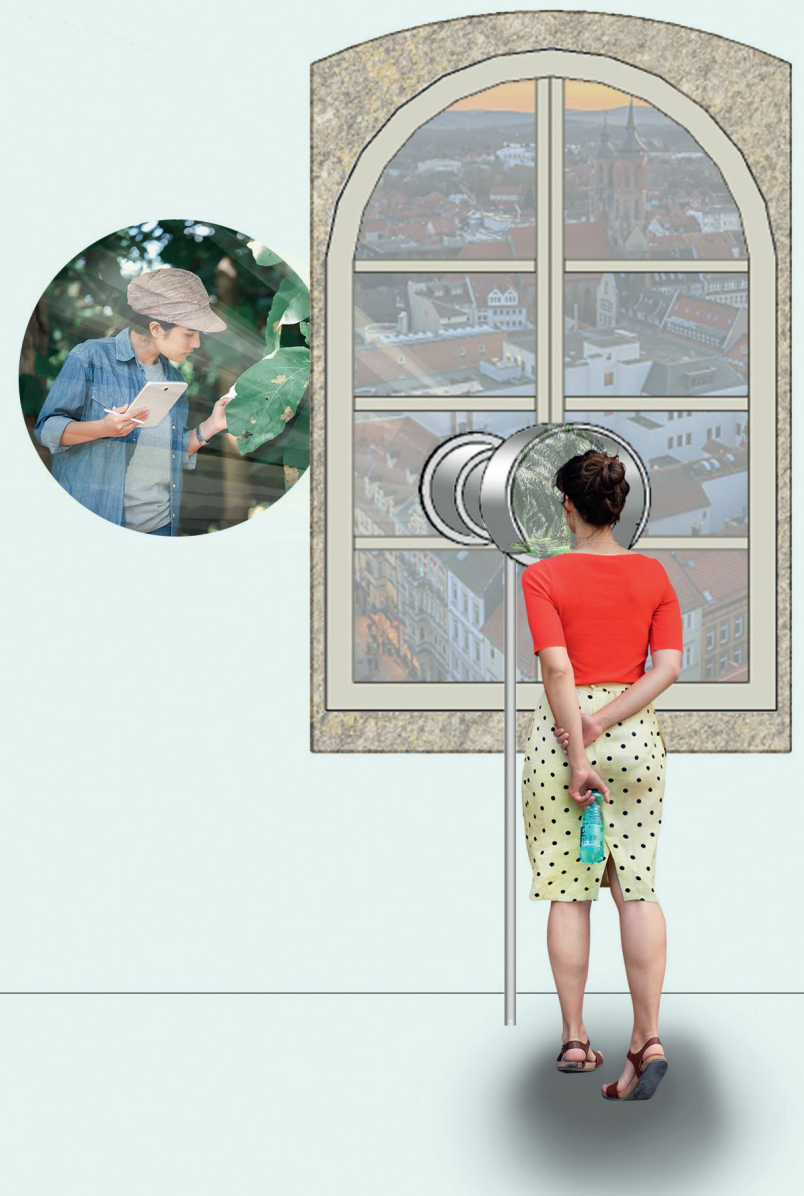

Beispiel 4:

Ein Fenster, welches die „versteckte" biologische Diversität innerhalb von Städten zeigt. 


\section{Das Gefühl des Staunens}

Die wenigsten Menschen besuchen eine Ausstellung allein. Deshalb ist es wichtig, dass Gruppen die Ausstellung gemeinsam erleben können, um ein kollektives Erlebnis zu ermöglichen.

In Familien mit Kindern übernehmen Eltern und/oder Großeltern in der Regel die Rolle der Betreuer für die kleineren Kinder, während ältere Kinder oder Teenager oft als eine Art "digital guide" agieren. Textinformationen müssen daher leicht lesbar und übersichtlich gestaltet sein, und die Exponate und Interaktionen sollten für alle zugänglich sein. Speziell für die ganz kleinen Kinder ist der "Tiny Explorers"-Bereich in jedem Kapitel gestaltet. In diesem Bereich gibt es themenbezogene Spiele und Interaktionen, die Kinder selbständig nutzen können. Darüber hinaus wird das wiederkehrende Spiel „Ich sehe was, was du nicht siehst" die Familien durch die Ausstellung begleiten, bei dem Kinder und Eltern versuchen können ein Detail im Raum, wie zum Beispiel eine Pflanze oder ein Tier, zu entdecken. Für Schulklassen werden wir eine Rallye durch die Ausstellung mit Stift-und-Papier oder in digitaler Form entwickeln.
Zusätzlich werden andere didaktische Ressourcen wie Handy-Apps zur Verfügung stehen, die mit den anderen Ausstellungen im Gebäude, wie dem "Forum Wissen", verknüpft sind.

Für alle - Alt oder Jung, mit oder ohne Einschränkung verstärkt das Ansprechen und die Aktivierung verschiedener Sinne das Erleben der Ausstellung.

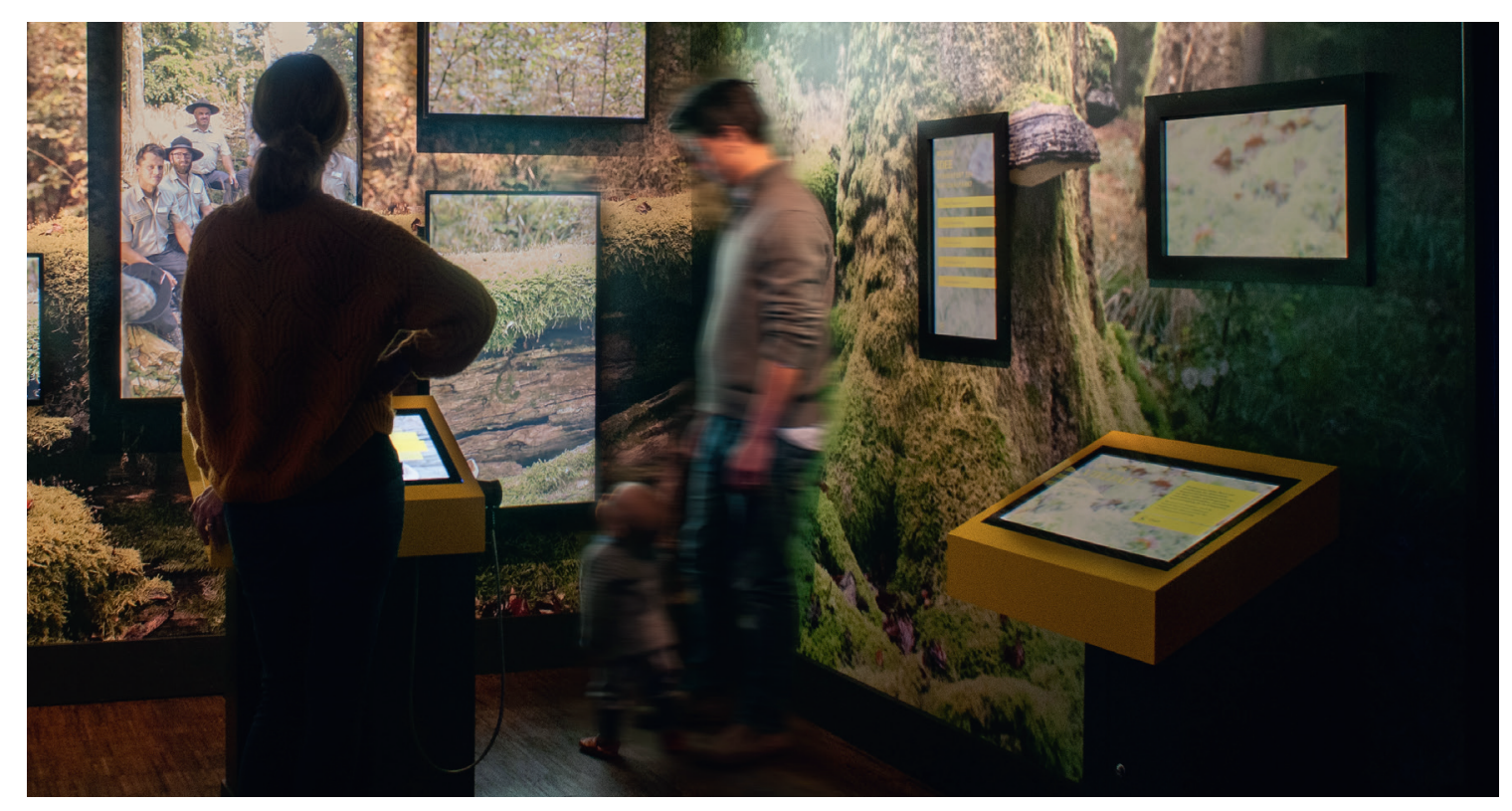



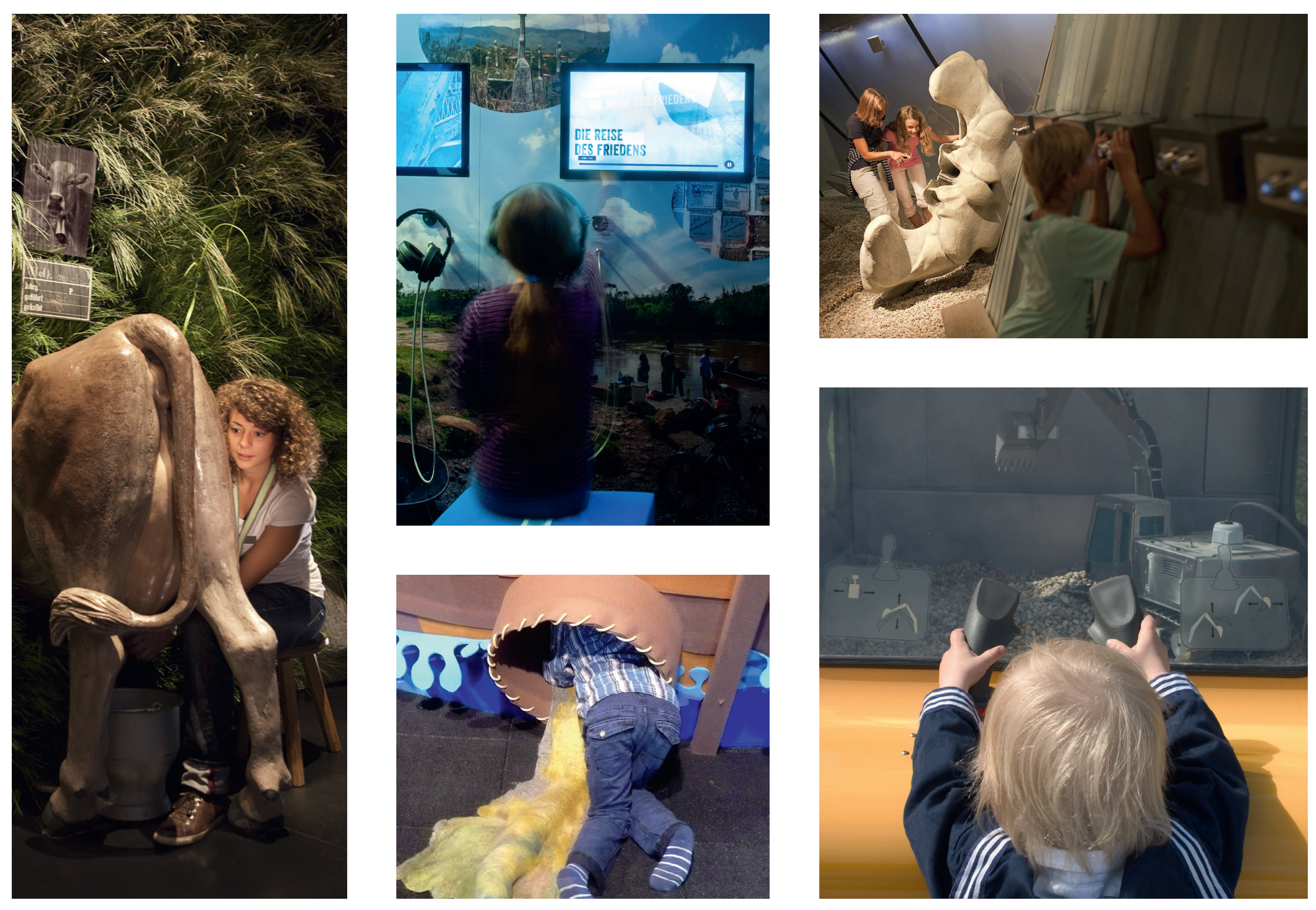


\section{Wissenschaftliche Inhalte}

Neben der Direktion und dem Kuratorium gibt es den wissenschaftlichen Beirat des Museums, ein Gremium aus (bisher) zwölf Wissenschaftler*innen, die an der Georg-August-Universität Göttingen forschen und lehren.

In dieser Gruppe finden sich Spezialist*innen in den Bereichen Biodiversität und Evolution der Tiere, Pflanzenund Tierökologie und Agrar- und Waldökologie, um nur einige zu nennen. Sie haben die Entwicklung dieses Konzeptes bisher beratend begleitet und werden das Projekt auch in der Realisierungsphase weiterhin tatkräftig unterstützen. Jedes Kapitel stellt einen oder mehrere Wissenschaftler*innen vor, so dass Besucher einen Einblick in echte wissenschaftliche Arbeit erhalten.

\section{Direktion und Kuration}

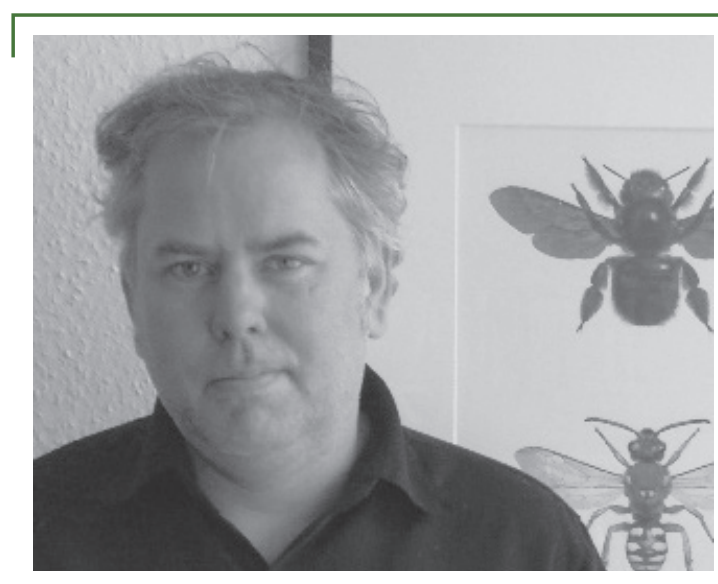

Prof. Dr. Christoph Bleidorn Fakultät für Biologie \& Psychologie

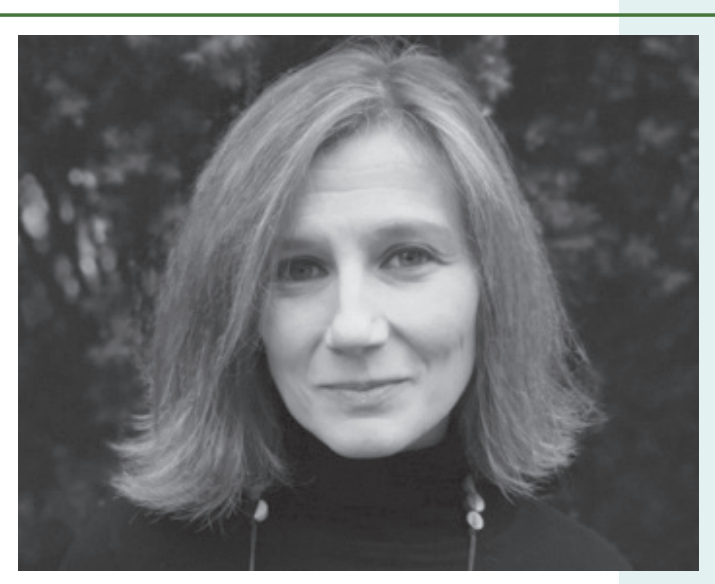

Dr. Maria Teresa Aguado Molina Fakultät für Biologie \& Psychologie 


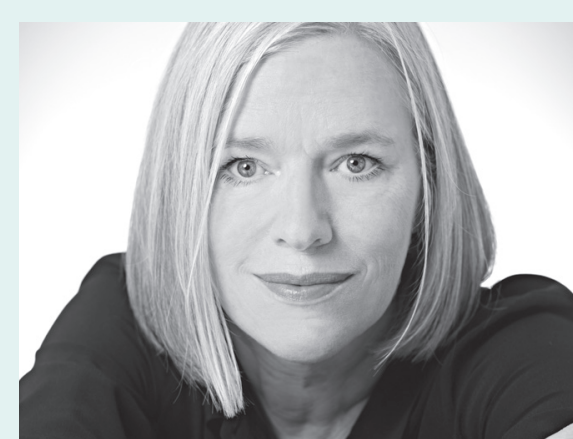

Prof. Dr. Julia Fischer

Fakultät für Biologie \& Psychologie

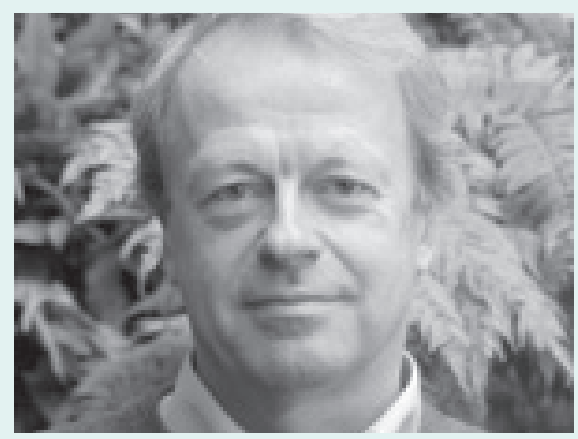

Prof. Dr. Christoph Leuschner Fakultät für Biologie \& Psychologie

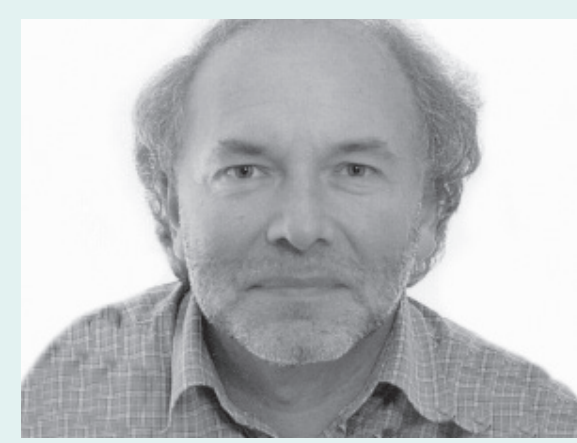

Prof. Dr. Hermann Behling Fakultät für Biologie \& Psychologie

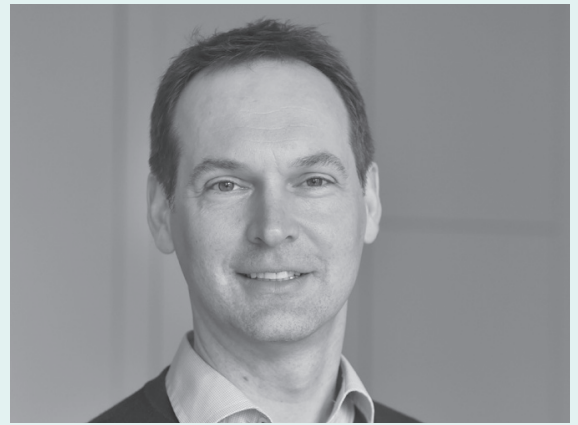

Prof. Dr. Alexander Knohl

Fakultät für Forstwissenschaften \& Waldökologie

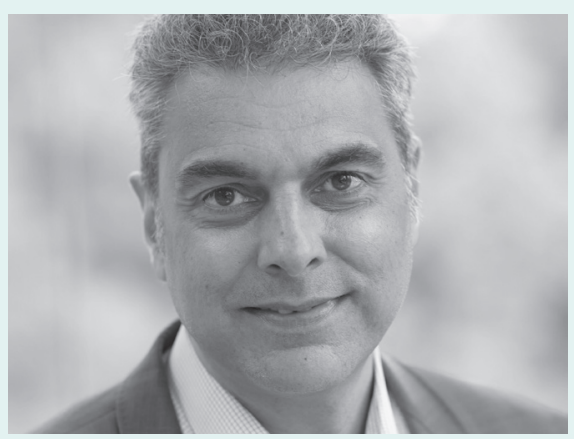

Prof. Dr. Matin Qaim

Fakultät für Agrarwissenschaften

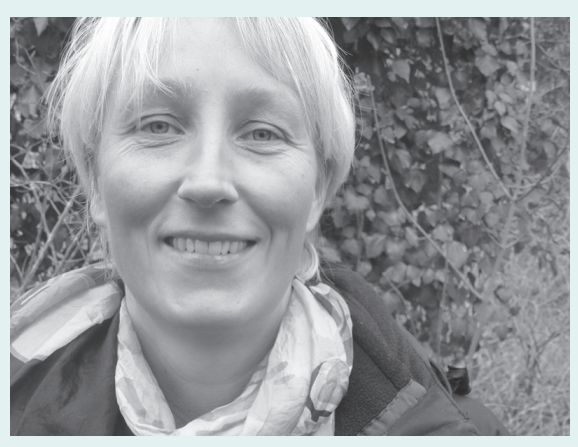

Dr. Maike Lorenz

Fakultät für Biologie \& Psychologie

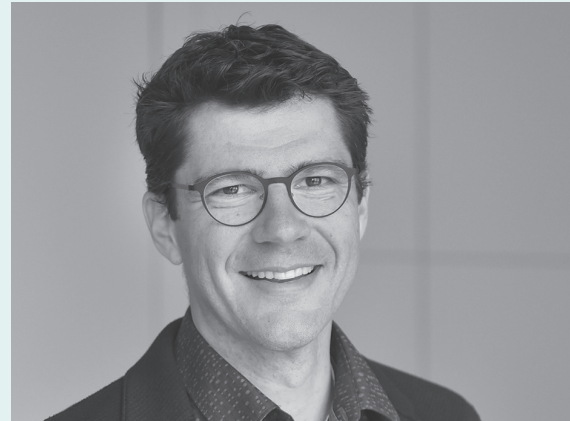

Prof. Dr. Holger Kreft

Fakultät für Forstwissenschaften \& Waldökologie

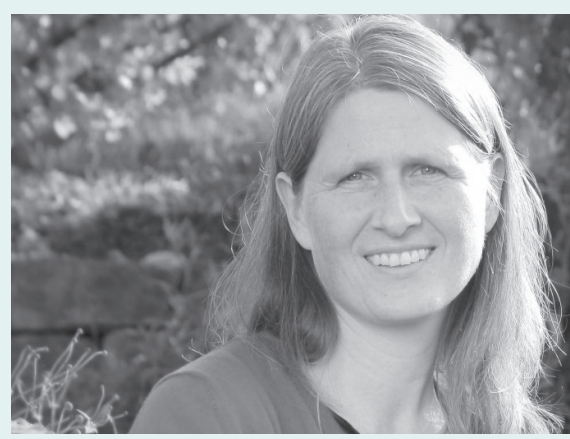

Prof. Dr. Catrin Westphal

Fakultät für Agrarwissenschaften

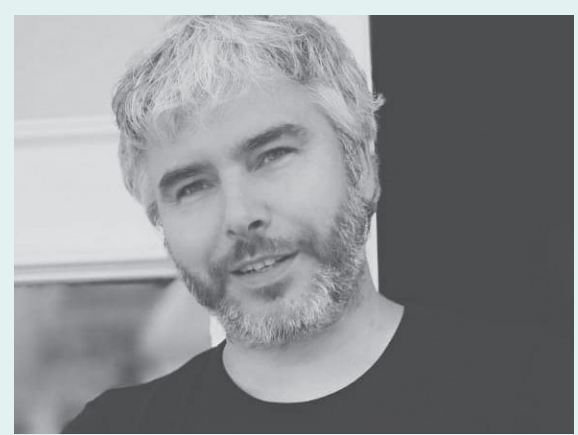

Dr. Sven Bradler

Fakultät für Biologie \& Psychologie

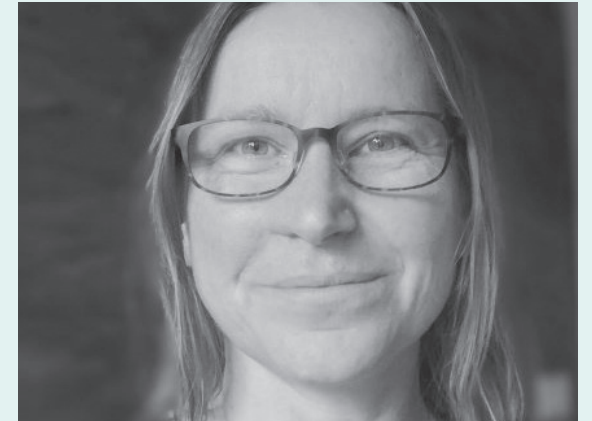

Dr. Simone Pfeiffer

Zentrum für Biodiversität \& Nachhaltige Landnutzung

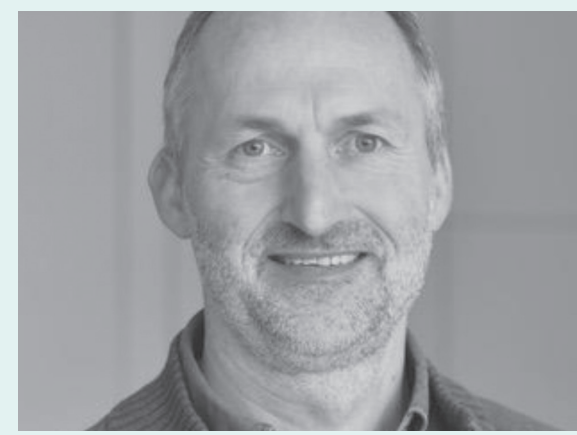

Prof. Dr. Stefan Scheu

Fakultät für Biologie \& Psychologie

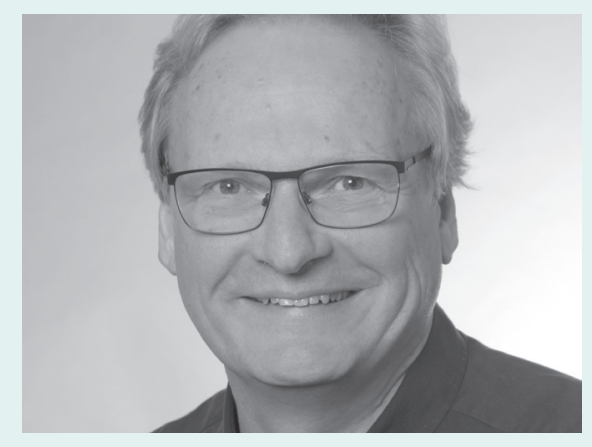

Prof. Dr. Thomas Friedl Fakultät für Biologie \& Psychologie 


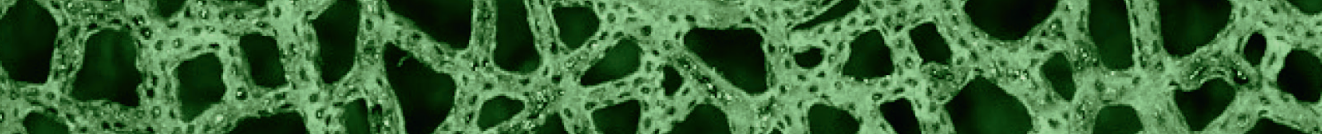

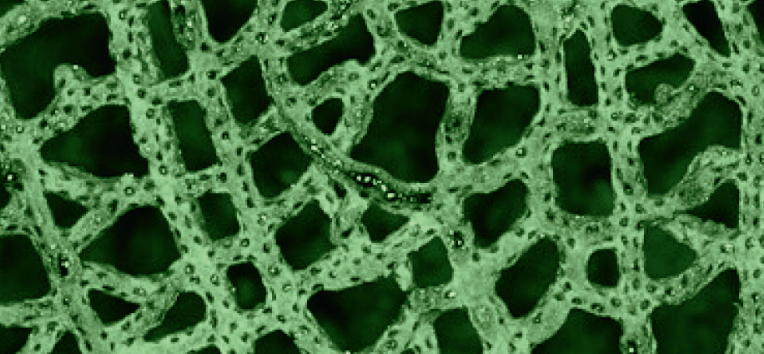

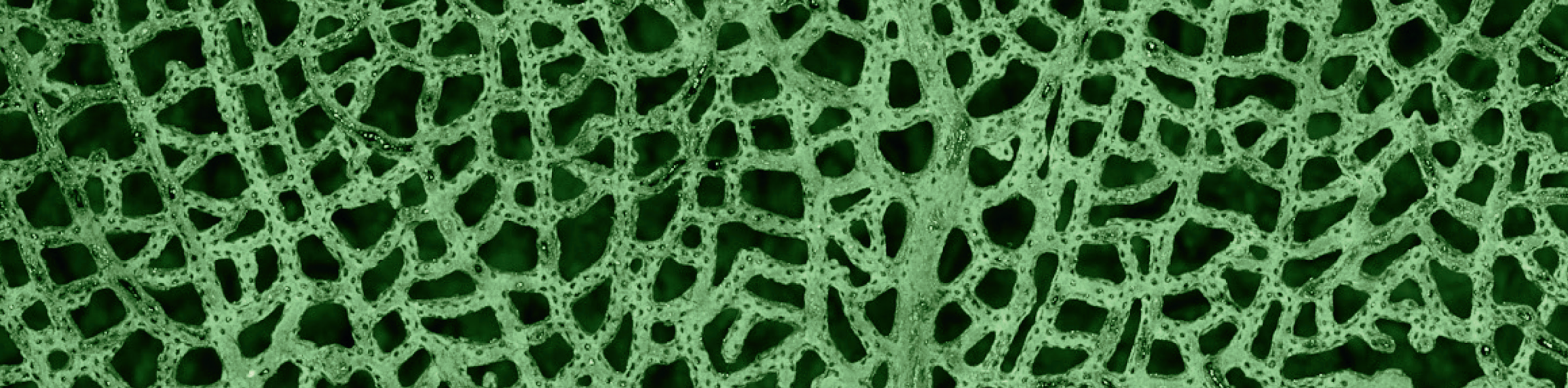

201

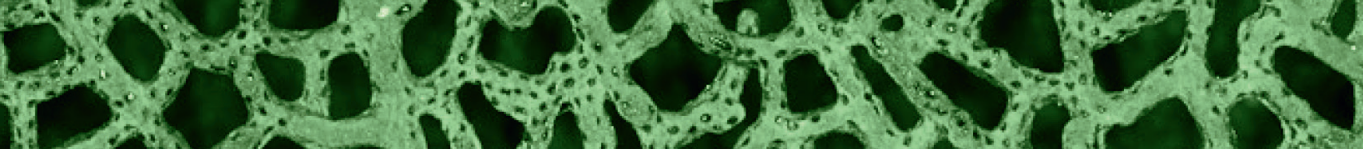

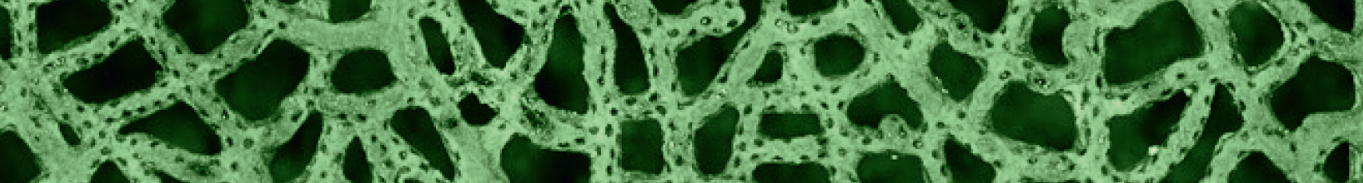

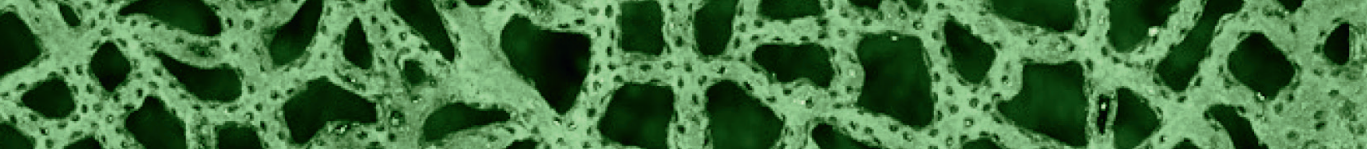

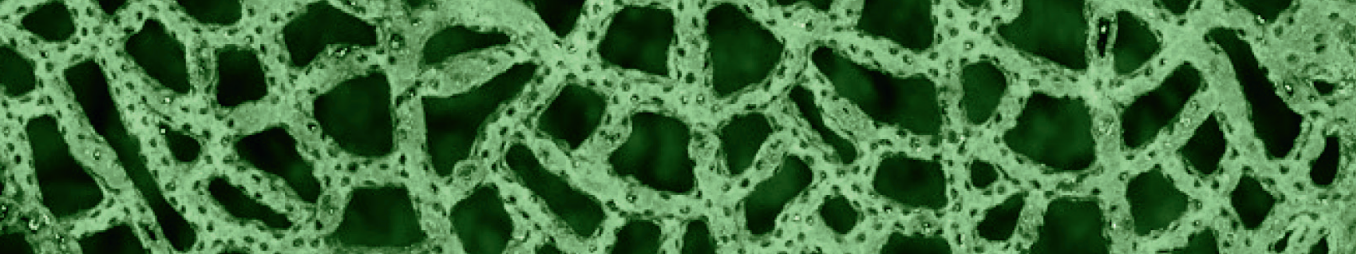

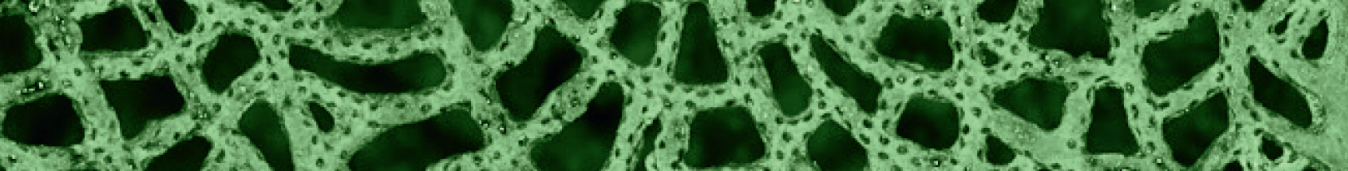

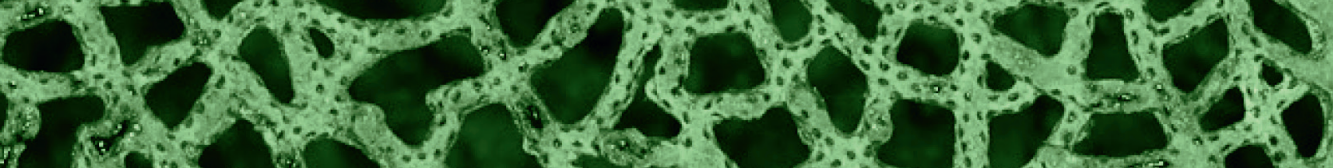

(SW

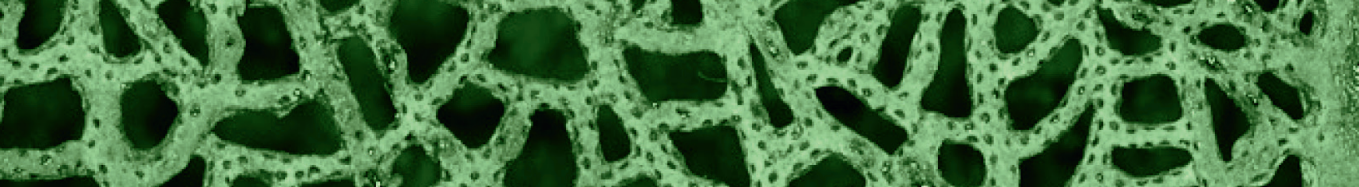

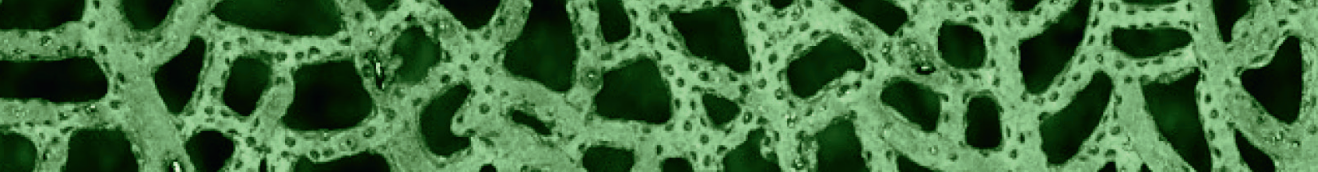

W The

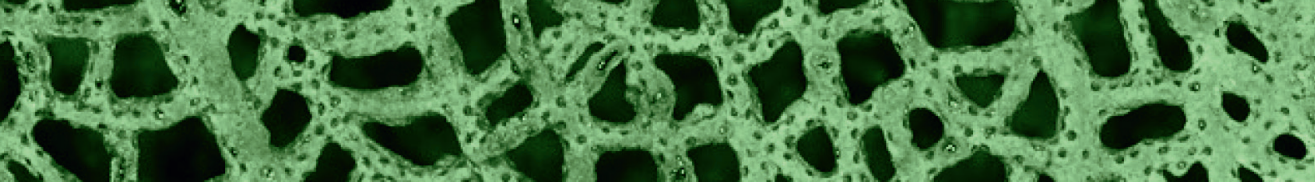

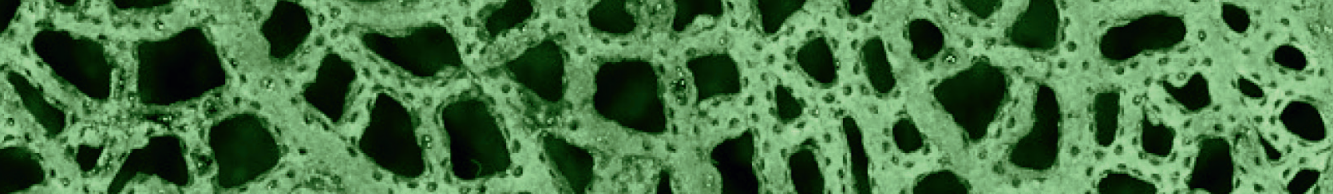

(to

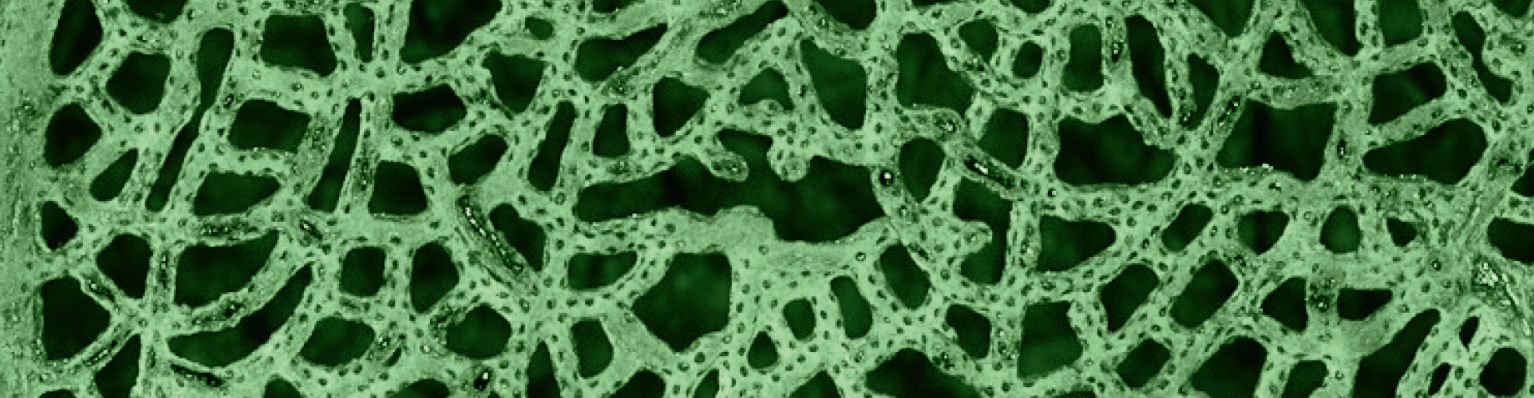

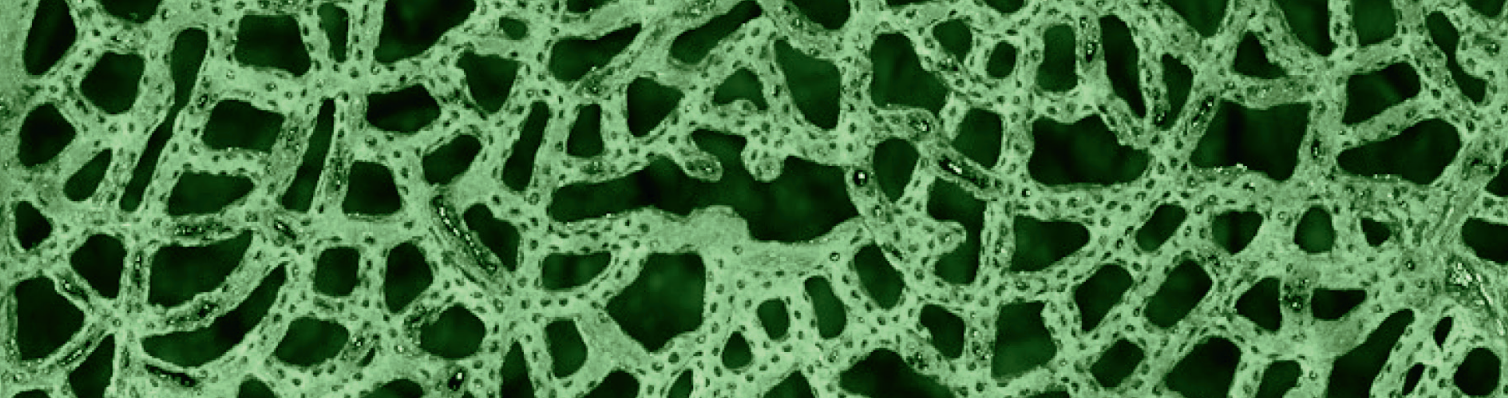

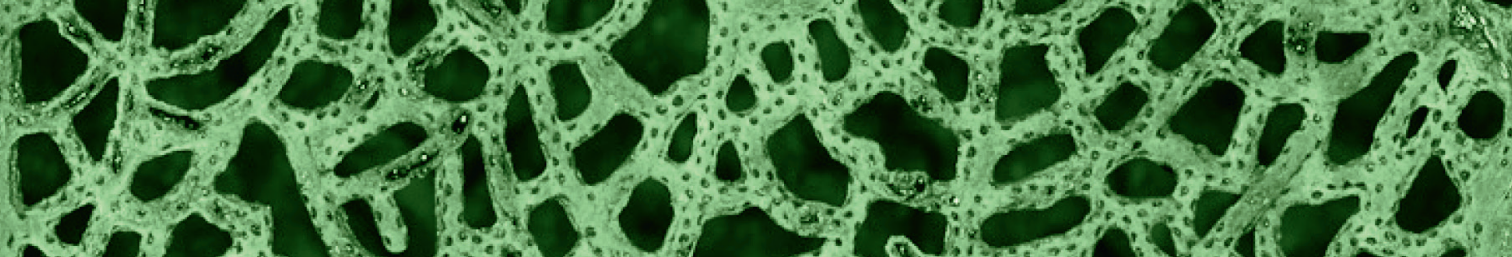

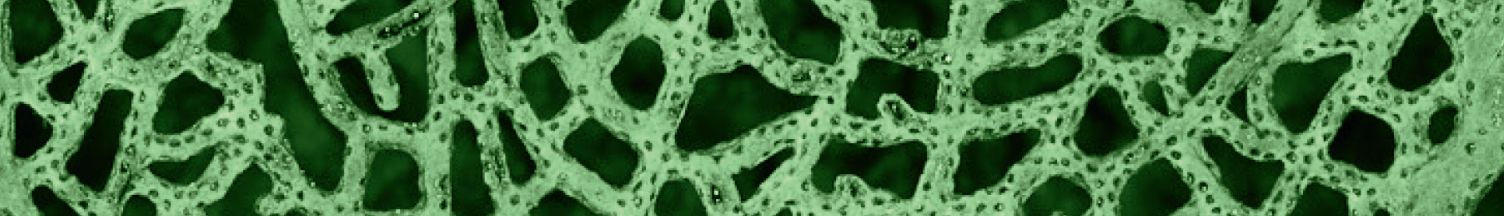

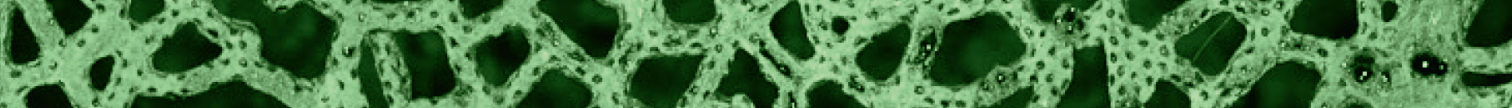

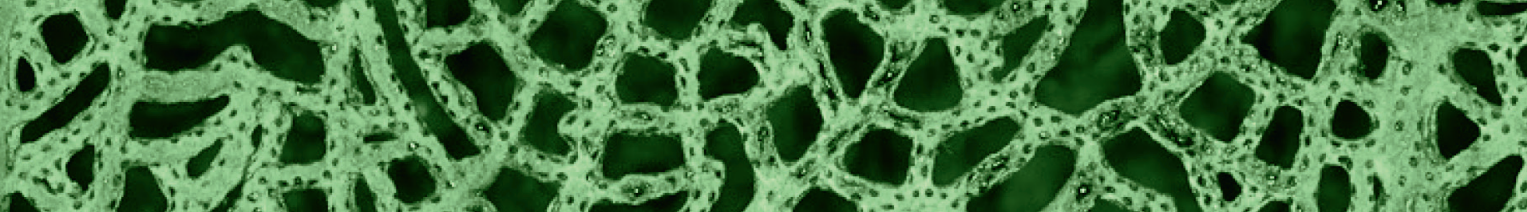
W.

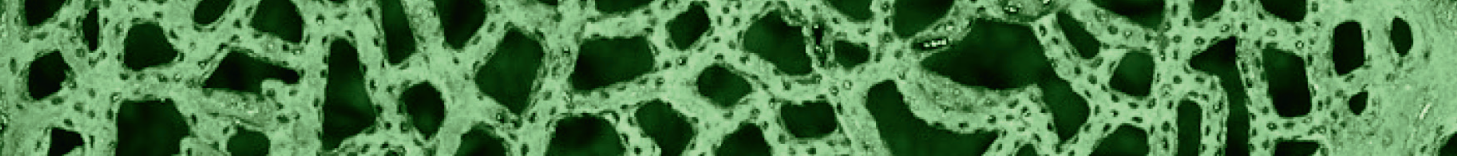

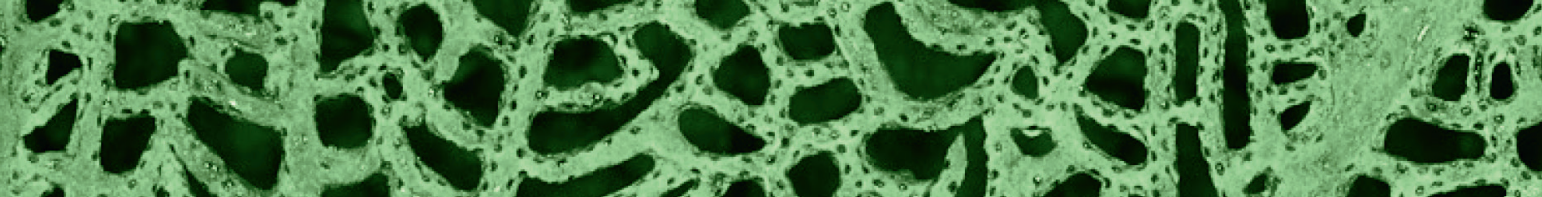

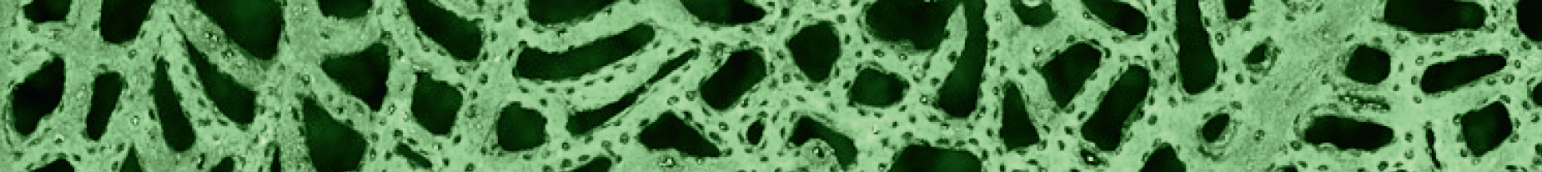

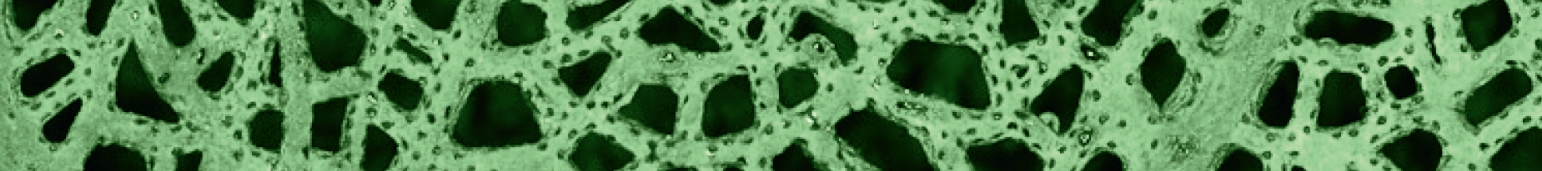

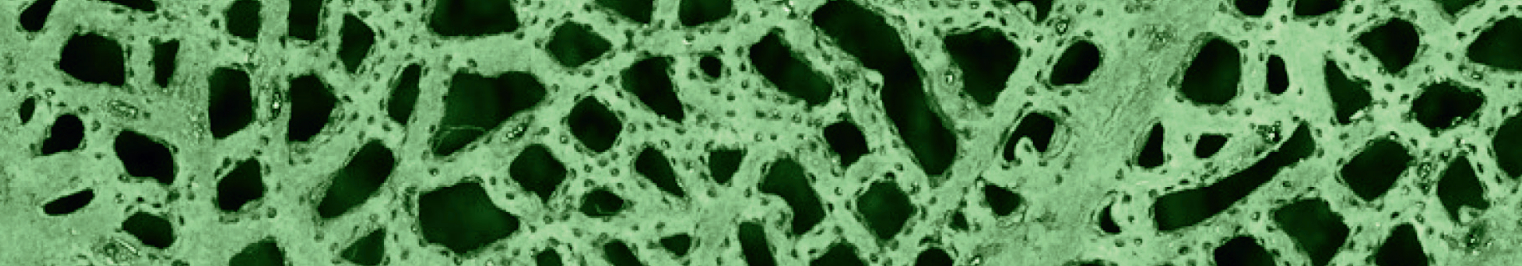

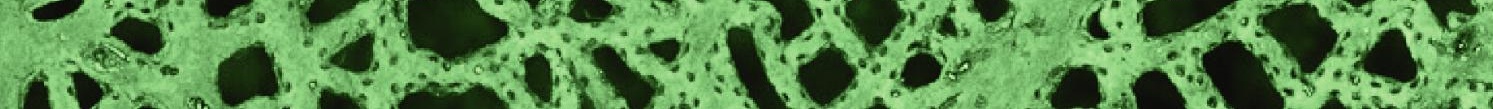




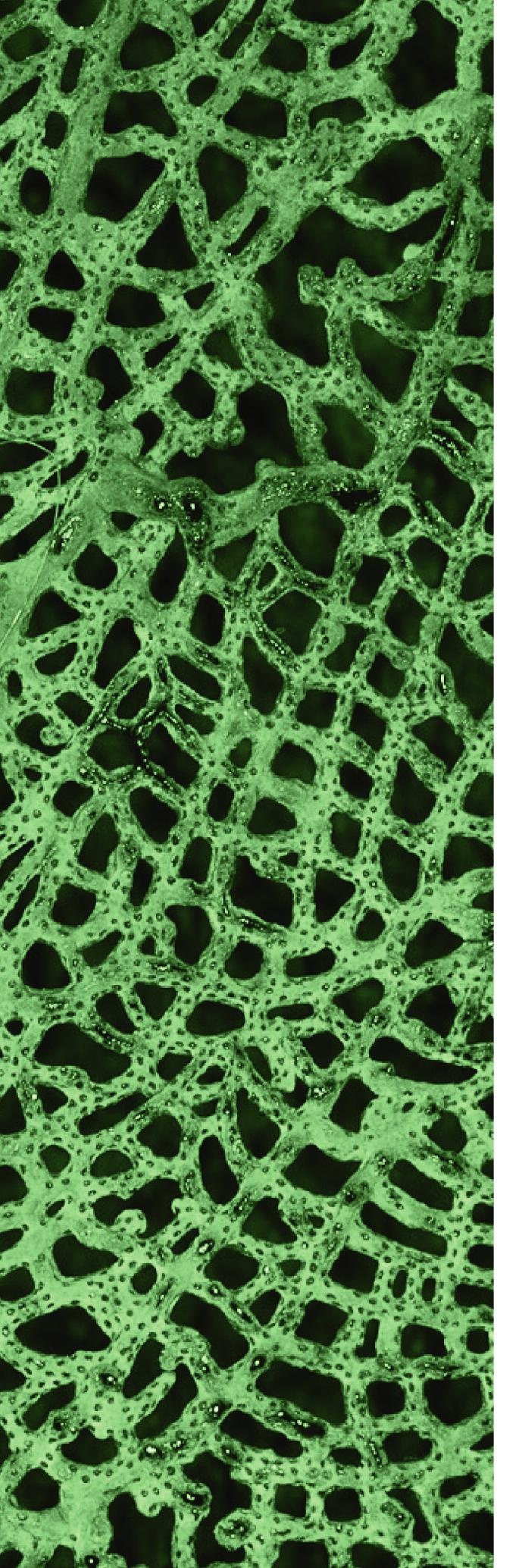

«Im weitesten Sinne könnte man unter Biodiversität das gesamte Leben auf der Erde verstehen, und alles, was am Leben vielfältig ist - Ökosysteme, Arten, Gene.»

- Georgina Mace

\section{Gang durch die Ausstellung}




\section{Prolog: Was ist Biodiversität?}

Der Prolog führt in das Thema der Ausstellung ein, liefert den Besucher*innen eine Definition für Biodiversität und gibt einen ersten Einblick in die Vielfalt der Natur.

Man betritt einen abgedunkelten Raum, in dem die drei Säulen der Biodiversität stehen (1. Vielfalt innerhalb von Arten (Genotypen und Phaenotypen), 2. Vielfalt der Arten, 3. Vielfalt der Ökosysteme). Wenn die Besucher*innen sich ihnen nähern, können sie leise Stimmen vernehmen, die Fragen stellen wie "Warum brauchen wir Biodiversität? Warum ist es wichtig, Biodiversität zu verstehen? Welchen Wert hat sie?". Auf einigen Monitoren werden Videos von anderen Besucher*innen gezeigt, die diese Fragen beantworteten, kurz bevor sie die Ausstellung betreten haben. Im Epilog der Ausstellung werden den Besucher*innen diese Fragen nochmal gestellt. So wird deutlich, ob und wie sich ihr Wissen und ihre Meinung über Biodiversität und Erhaltungsmaßnahmen verändert hat.

Jede der drei transparenten Säulen repräsentiert einen Aspekt der Biodiversität: die Diversität von Ökosystemen, die interspezifische Diversität und die intraspezifische Diversität.

\section{Themen:}

- Definition von Biodiversität und deren Veranschaulichung

- Grundlegende Fragen zur Bedeutung der Biodiversität, die im Laufe der Ausstellung beantwortet werden

\section{Schlüsselexemplare:}

- Erste Säule (Artenvielfalt): Nasspräparate zur Darstellung der Artenvielfalt

- Zweite Säule (Vielfalt innerhalb einer Art): Genotypen und Phaenotypen; eine große Anzahl von Schneckengehäusen der gleichen Art, die die phänotypische (und genotypische) Vielfalt illustriert - Dritte Säule (Vielfalt der Ökosysteme): Modelle, die verschiedene Ökosysteme zeigen, wie Regenwald, Wüste oder alpines Ökosystem 


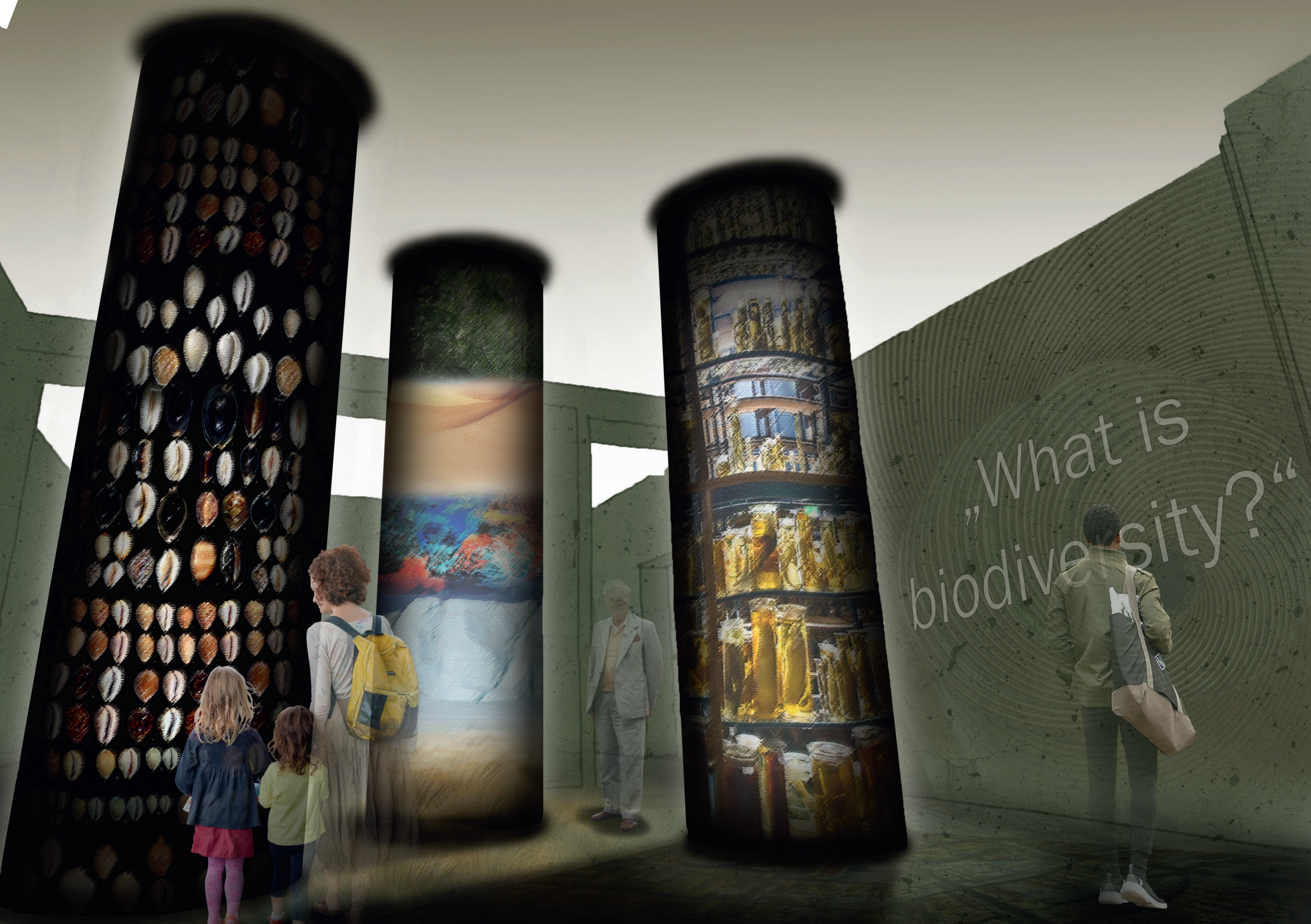






\section{Szenografie \& Motive}

In diesem Kapitel der Ausstellung wird die Grundlage für das Verständnis der Evolutionsbiologie geschaffen, indem veranschaulicht wird, wie Leben und Umwelt zusammenwirken.

Der Raum mag für Besucher*innen, die Vielfalt erwarten, erstmal überraschend sein: abgedunkelt und eher karg ausgestattet, mit einer Gruppe von beleuchteten Felsen im Mittelpunkt. Die Wände sind mit Flechtenreproduktionen bedeckt, während die Felsen mit echten Flechten überwachsen sind. Man bekommt den Eindruck, eine solche Umgebung sei scheinbar lebensfeindlich. Doch wie die Besucher*innen erfahren, ist der mit Flechten bedeckte Felsen eines der kleinsten kompletten Ökosysteme.

\section{Kristall: Schlüsselexemplare}

Flechten und flechtenfressende Mottenlarven (zum Beispiel Tigermottenarten der Gattung Coscinia)
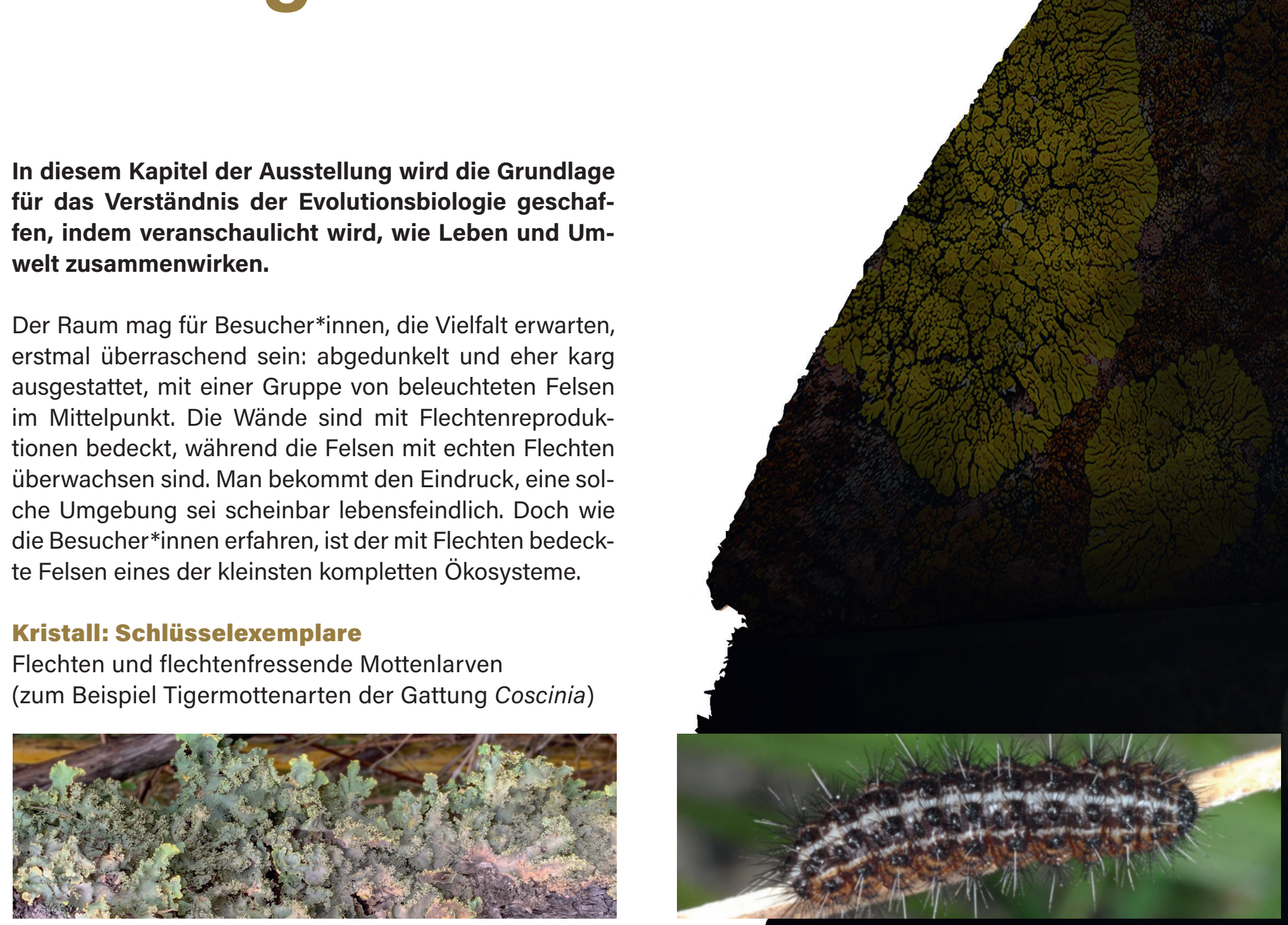

Die flechtenfressende Mottenlarve 


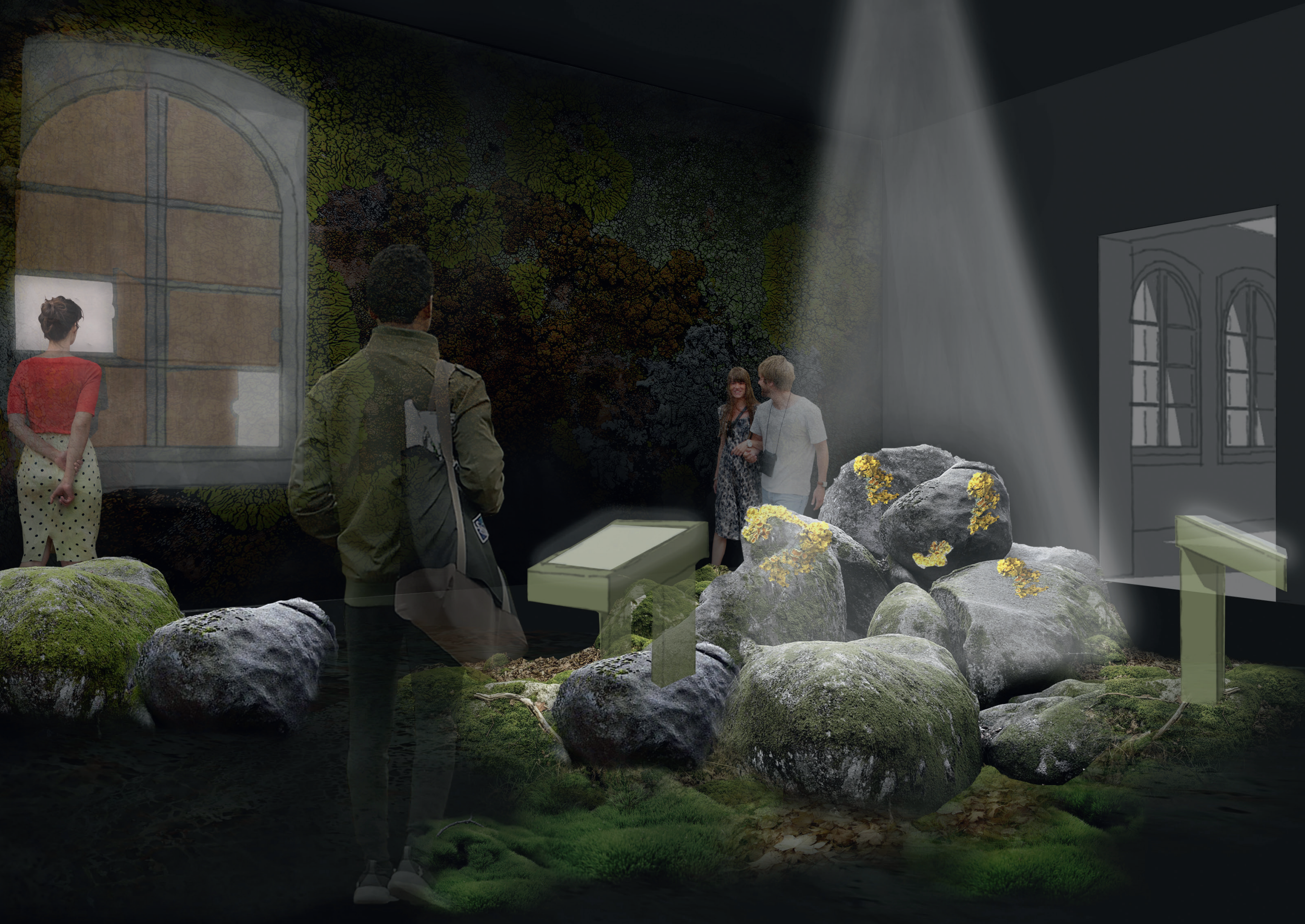




\section{Themen \& Interaktionen}

Ring 1: interaktive Stationen

- Interaktive Station: Definition von Ökosystem

- Interaktive Station: Ökologische Nische

- Interaktive Station: Symbiose

Ring 2: interaktive Station

Eine Station, an der Flechten unter dem Mikroskop betrachtet werden können. Für Schüler*innen eine zusätzliche Aufgabe geben, bei der sie verschiedene Flechtengruppen identifizieren.

\section{Ring 3: Fensterelemente}

Erst vor etwa 150 Jahren erkannten Wissenschaftler, dass Flechten aus verschiedenen Organismen zusammengesetzt sind: Pilze und Algen.

\section{Tiny Explorers:}

Flechtenpuzzle mit legoartigen Bausteinen, bei dem sie verschiedene Formen zusammensetzen und eine 3D-Flechte bauen können.

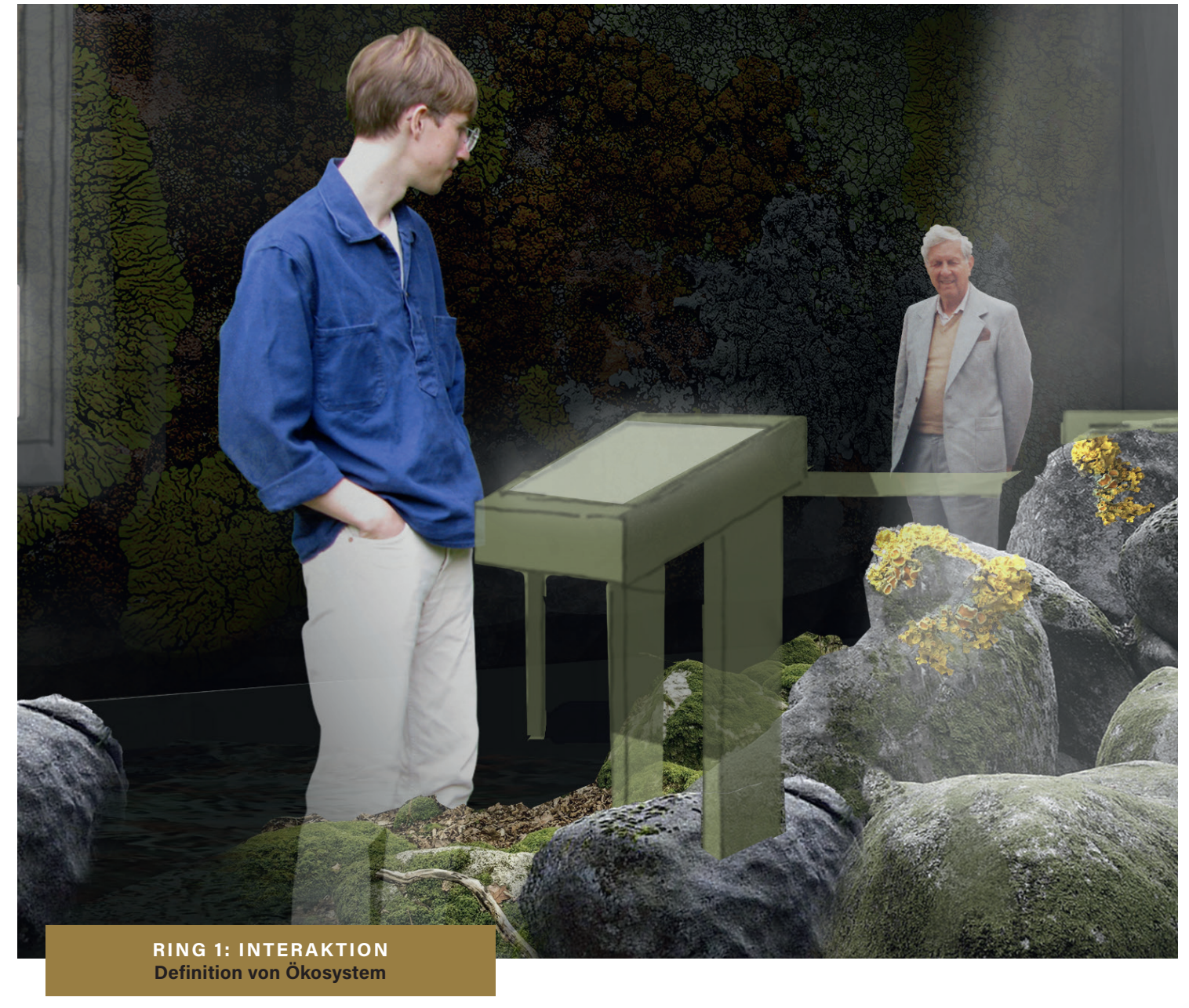



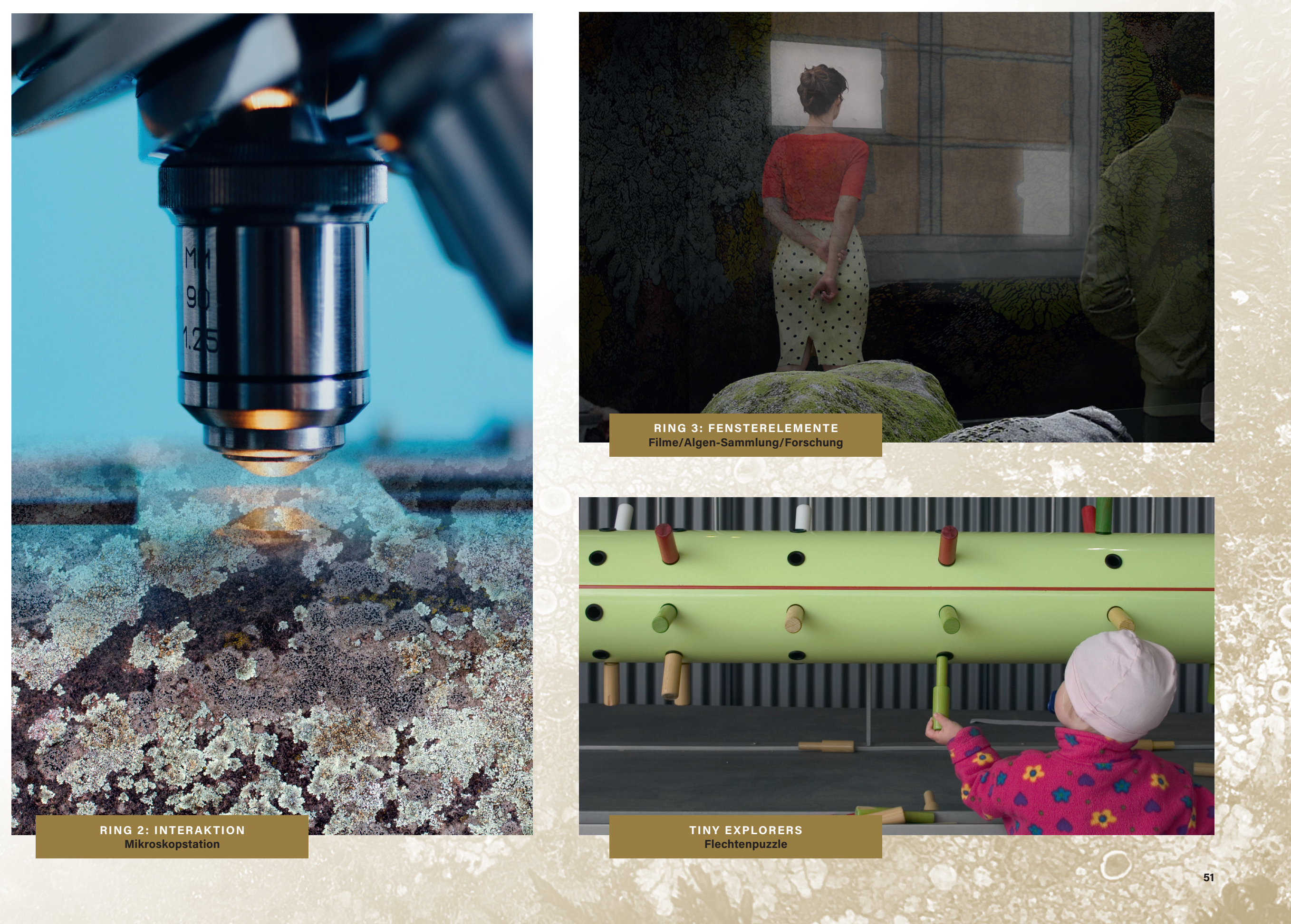


\section{Räumliche Aufteillung}

\section{Dr. Maike Lorenz}

Maike Lorenz ist die Kuratorin der Sammlung von Algenkulturen (SAG) und seit 1999 in der Abteilung für Experimentelle Phykologie und Sammlung von Algenkulturen sowie Dozentin am Albrecht-von-Haller-Institut. Ihre Expertise liegt vor allem in der Isolation, Identifikation, Kultivierung und Kryokonservierung von Mikro- und Makroalgen und ihren möglichen biotechnologischen Anwendungen.

\section{Prof. Dr. Thomas Friedl}

Thomas Friedl ist seit 1999 Professor und Leiter der Abteilung Experimentelle Phykologie und Sammlung von Algenkulturen sowie der wissenschaftliche Direktor der Sammlung von Algenkulturen (SAG). Seine Forschungsschwerpunkte liegen in den Bereichen der Systematik, molekularen Phylogenie, Biodiversität und Biogeographie von terrestrischen Algen und Süßwasseralgen, Algen in komplexen mikrobiellen Systemen und den Potentialen der Algenbiodiversität als nachhaltige Ressourcen.

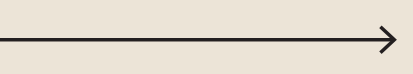




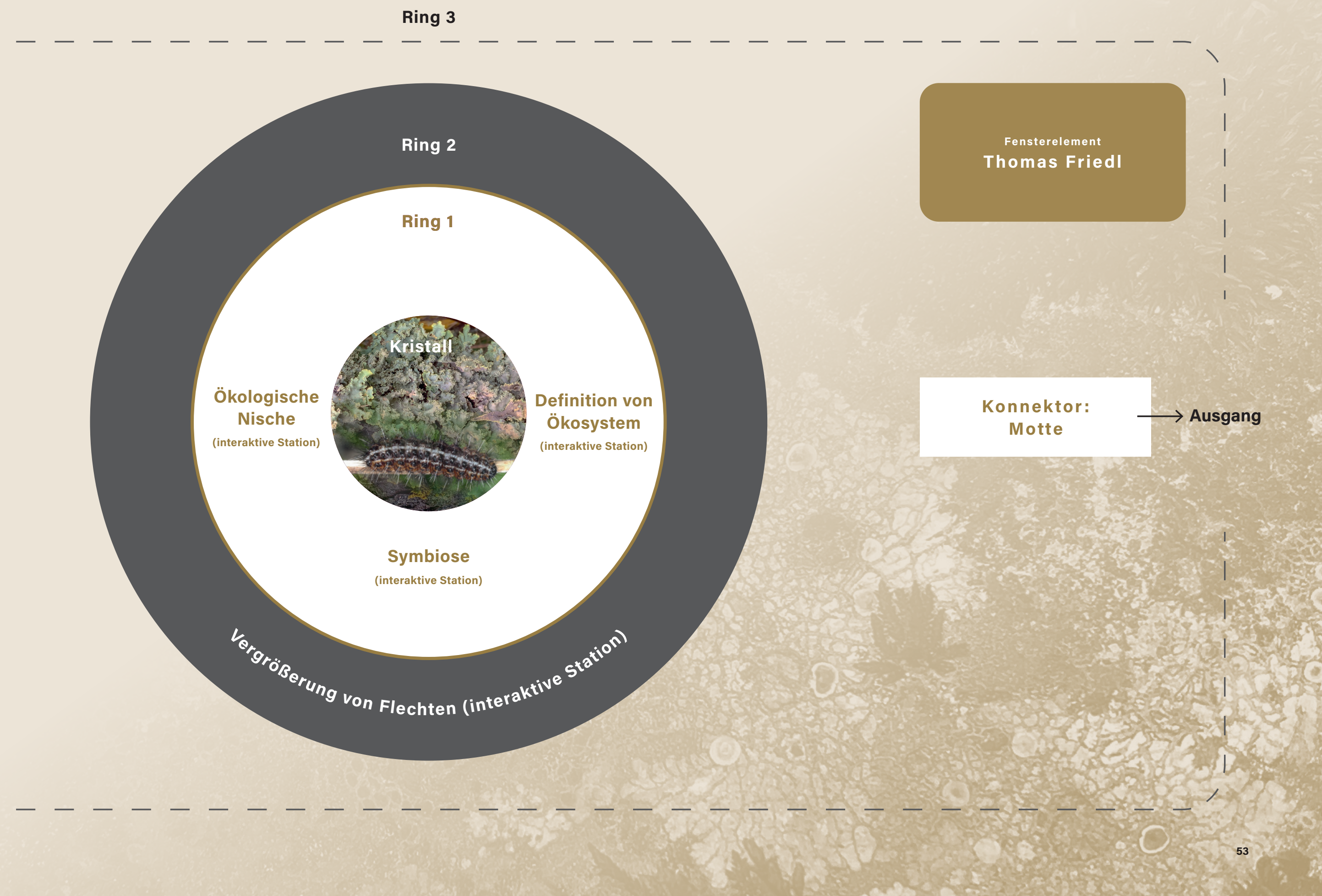




\section{Der Konnektor}

Flechten können auf einem Felsen leben, können aber auch Teil eines größeren Ökosystems sein.

Einige Raupen (Flechtenmotten) ernähren sich ausschließlich von Flechten. Stellvertretend wird eine dieser Arten aus der Gattung Coscinia hier vorgestellt. Nach der dargestellten Metamorphose folgen die Besucher*innen der Motte in den nächsten Raum. 


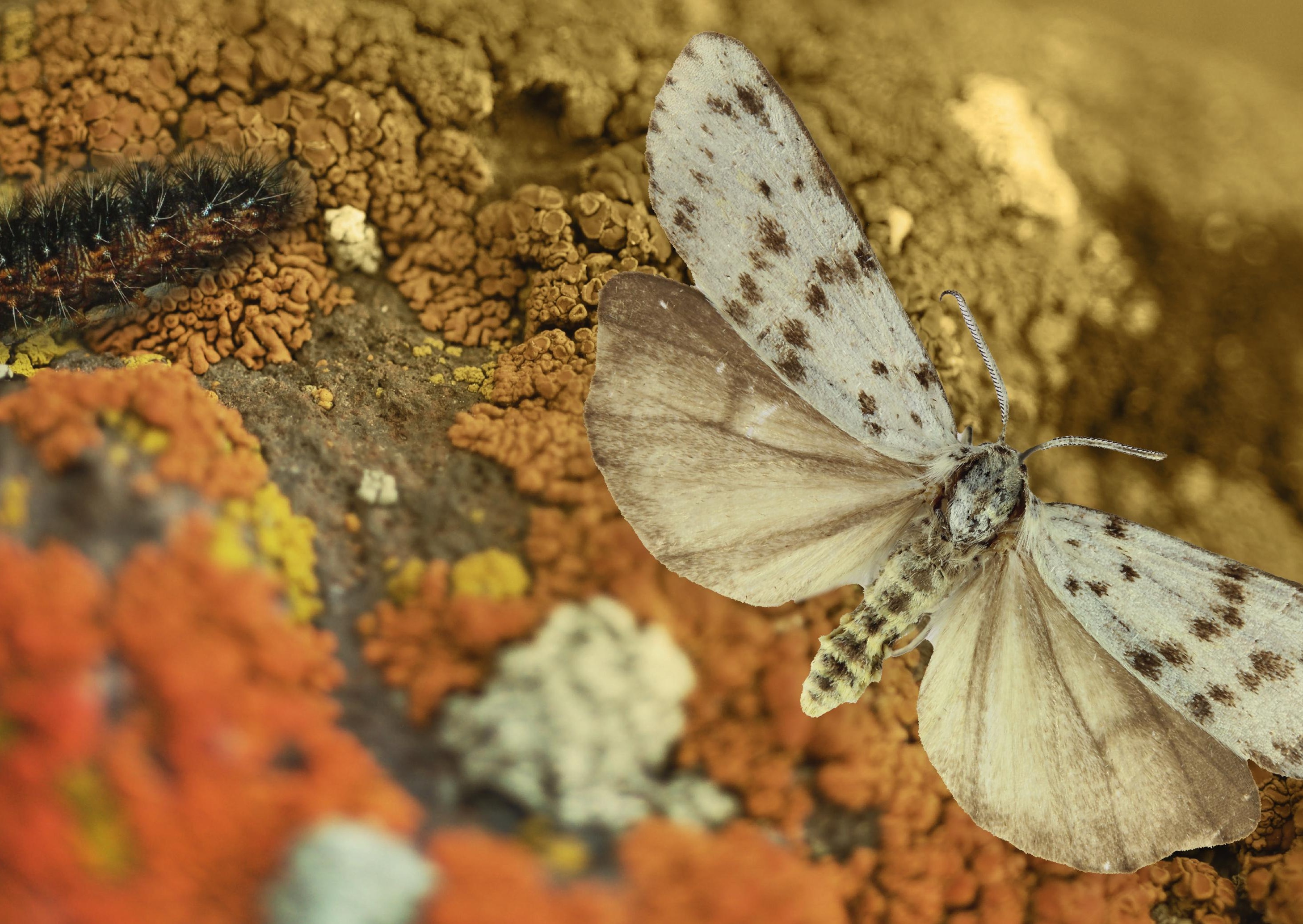




\section{Gang durch die Ausstellung}

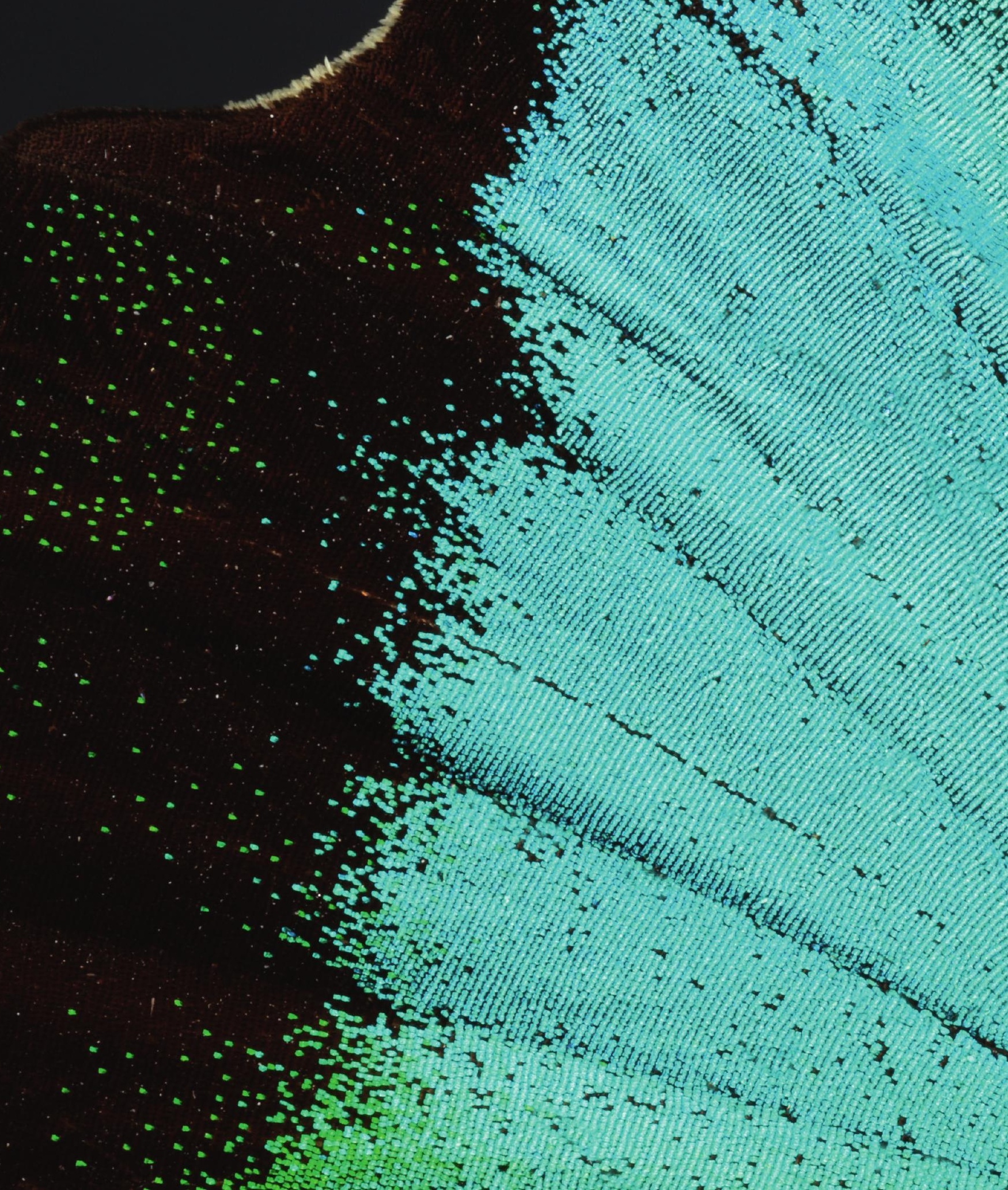




\section{Was ist eine Art?}

«Insekten sind die artenreichste Tiergruppe und stellen $70 \%$ aller Arten - damit sind sie zentraler Teil aller Ökosysteme. Wahrscheinlich wurden die meisten Insektenarten noch gar nicht entdeckt und haben noch nicht einmal einen Namen,»

- Teja Tscharntke

\section{Kapitel 2}




\section{Szenografie \& Motive}

In diesem Kapitel wird erklärt, was eine Art ist, wie Arten entstehen und wie sie von Wissenschaftler*innen untersucht und systematisiert werden.

Nachdem sie der Motte aus dem abgedunkelten Flechtenraum gefolgt sind, betreten die Besucher*innen nun einen Raum, der eine Fülle von Farben, Mustern und Formen zeigt. Der Kristall ist ein Mobile, welches die evolutionären Abstammungslinien der Insekten zeigt und in den Silhouetten der repräsentierten Gruppen endet.

Die Wände sind bedeckt mit vergrößerten Reproduktionen von Flügelmustern, Panzern, Facettenaugen und Fühlern von Insekten. Es gibt viele Insektenkästen mit hunderten von Exemplaren - Bienen und Wespen, Schmetterlinge,

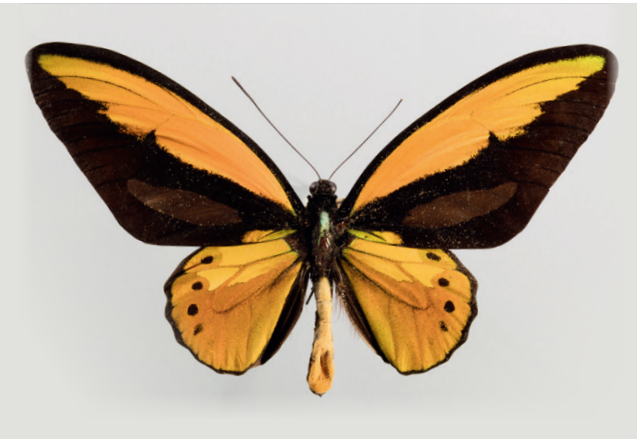

Ornithoptera croesus lydius (Felder \& Felder, 1865) Zoologische Sammlung / Universität Göttingen
Libellen und Käfer. In diesem Raum erfahren die Besucher*innen am Beispiel der artenreichsten Tiergruppe mehr über das Artkonzept und damit verwandte Themen. Sie können die Vielfalt bestaunen, Arten aus verschiedenen Gruppen und Habitaten vergleichen und beginnen die Komplexität der Evolution zu verstehen.

\section{Der Kristall:}

Mobile mit Schlüsselexemplaren zur Darstellung der Evolution von Insekten. Unterschiedlichste Insekten, und zwar in Massen!

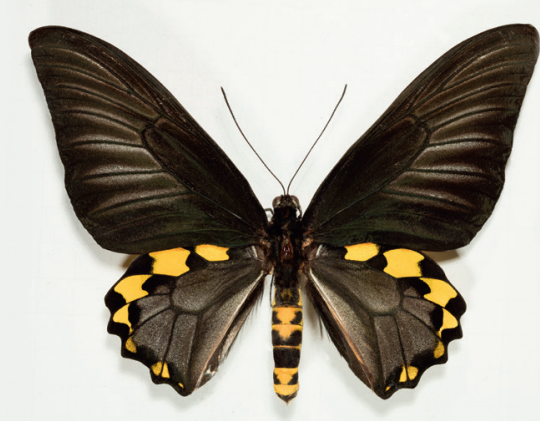

Troides hippolytus hippolytus (Cramer, 1775) Zoologische Sammlung / Universität Göttingen

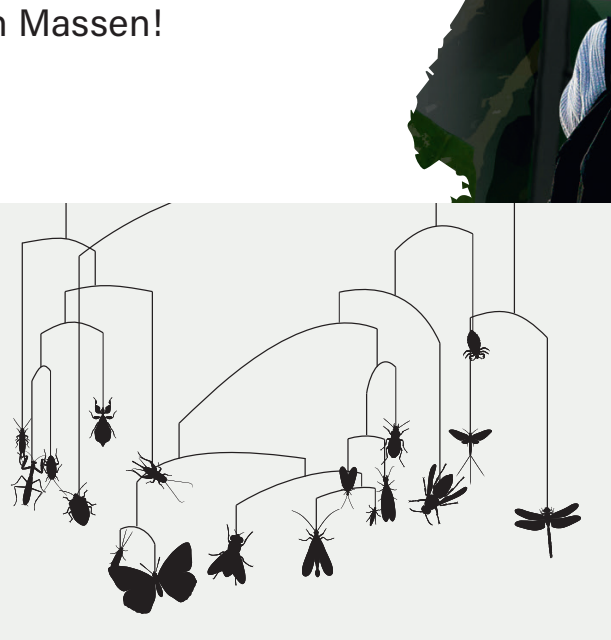

Der Kristall: Mobile mit Insekten 


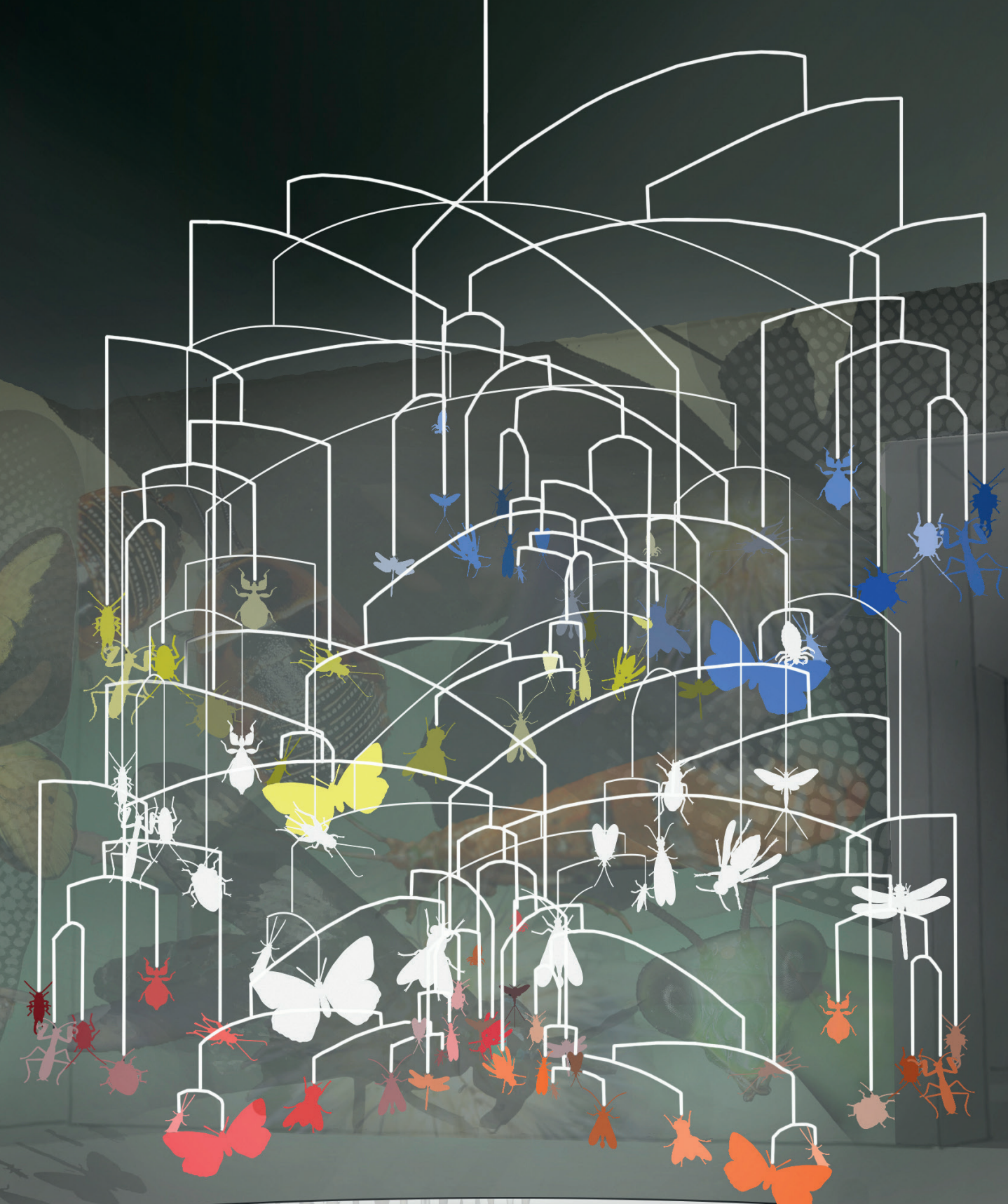




\section{Themen \& Interaktionen}

Ring 1: interaktive Stationen

- Definition von Arten

- Wie viele Arten gibt es und wie viele kennen wir?

- Insekten - die artenreichste Gruppe der Tiere

Ring 2: interaktive Stationen

- Definition von Evolution (einschließlich Variation)

- Wie entsteht genetische Vielfalt?

(Mutation, Rekombination, Genfluss, Hybridisierung)

- Besucher*innen können Hummeln anhand ihrer Farbmuster Gruppen zuordnen, um so zu verstehen, wie Systematisierung prinzipiell funktioniert.

Ring 3: Fensterelemente

- Natürliche Selektion und genetische Drift

- Systematik I - Warum ist es so wichtig, zu benennen und zu kategorisieren?

\section{Tiny Explorers:}

- „Ich sehe was, was du nicht siehst"-Spiel, bei dem sie bestimmte Insekten in den Vitrinen finden müssen.

- "Mach dein eigenes Insekt"-Spiel, bei dem die Kinder entweder Puzzleteile verwenden oder ein neues Insekt zeichnen.

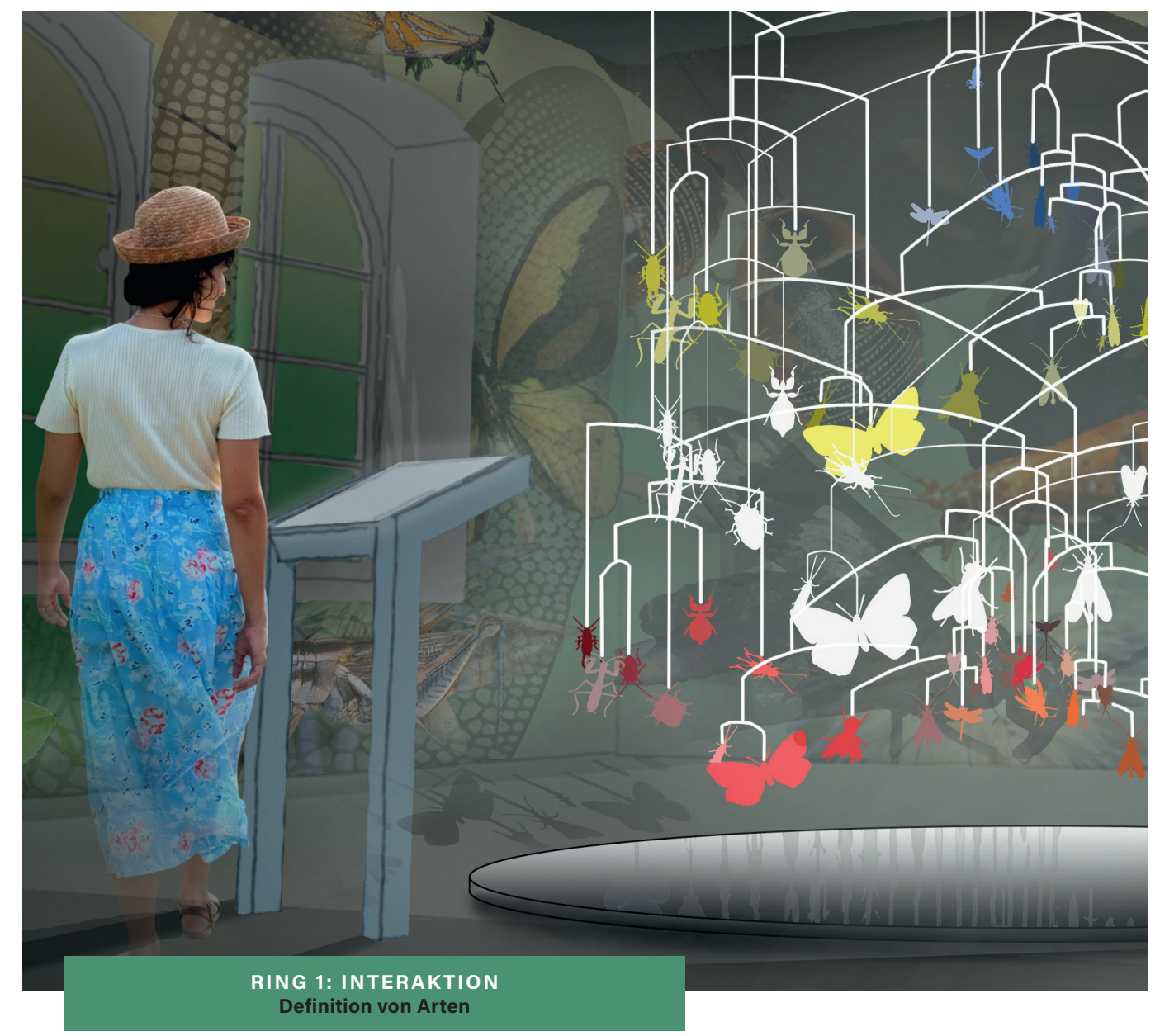




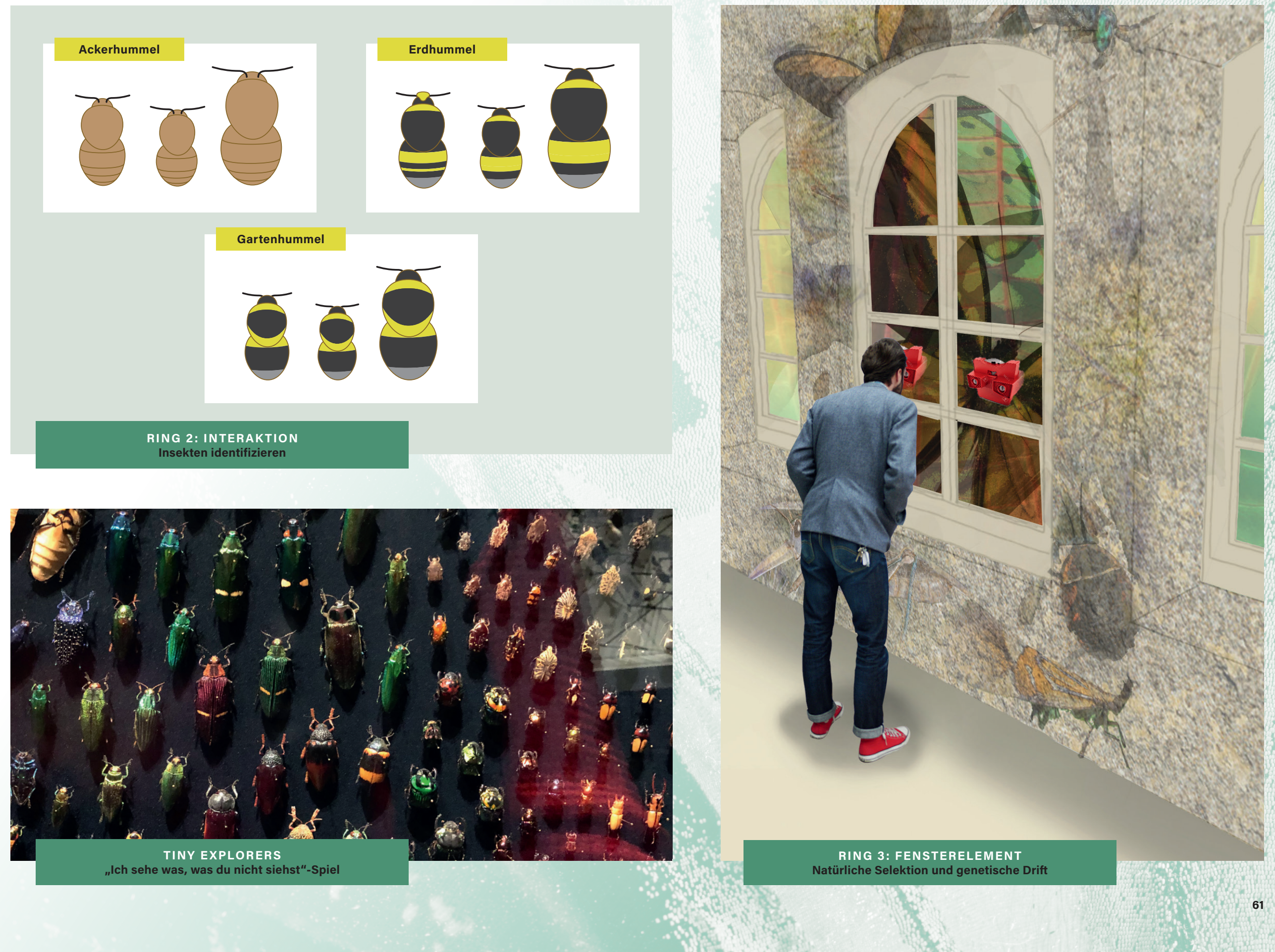




\section{Räumliche Aufteilung}

Dr. Sven Bradler

Sven Bradler ist wissenschaftlicher Mitarbeiter und Dozent in der Abteilung Evolution und Biodiversität der Tiere am Johann-Friedrich-Blumenbach-Institut. Seine wissenschaftliche Arbeit konzentriert sich auf die Erforschung makroevolutionärer Muster der taxonomischen und morphologischen Diversität und biogeografischer Verbreitung innerhalb eines phylogenetischen Rahmens.

\section{Prof. Dr. Catrin Westphal}

Catrin Westphal hat seit 2018 eine DFG-HeisenbergProfessur für Funktionelle Agrobiodiversität an der Fakultät für Agrarwissenschaften inne. Ihr Forschungsschwerpunkt liegt auf der Entwicklung und Erforschung innovativer ökologischer Intensivierungsverfahren, die die Produktivität, Agrobiodiversität und ihre vielfältigen Funktionen innerhalb landwirtschaftlicher Systeme erhalten und fördern.

\section{Eingang}

Fensterelement 


\section{Ring 3}

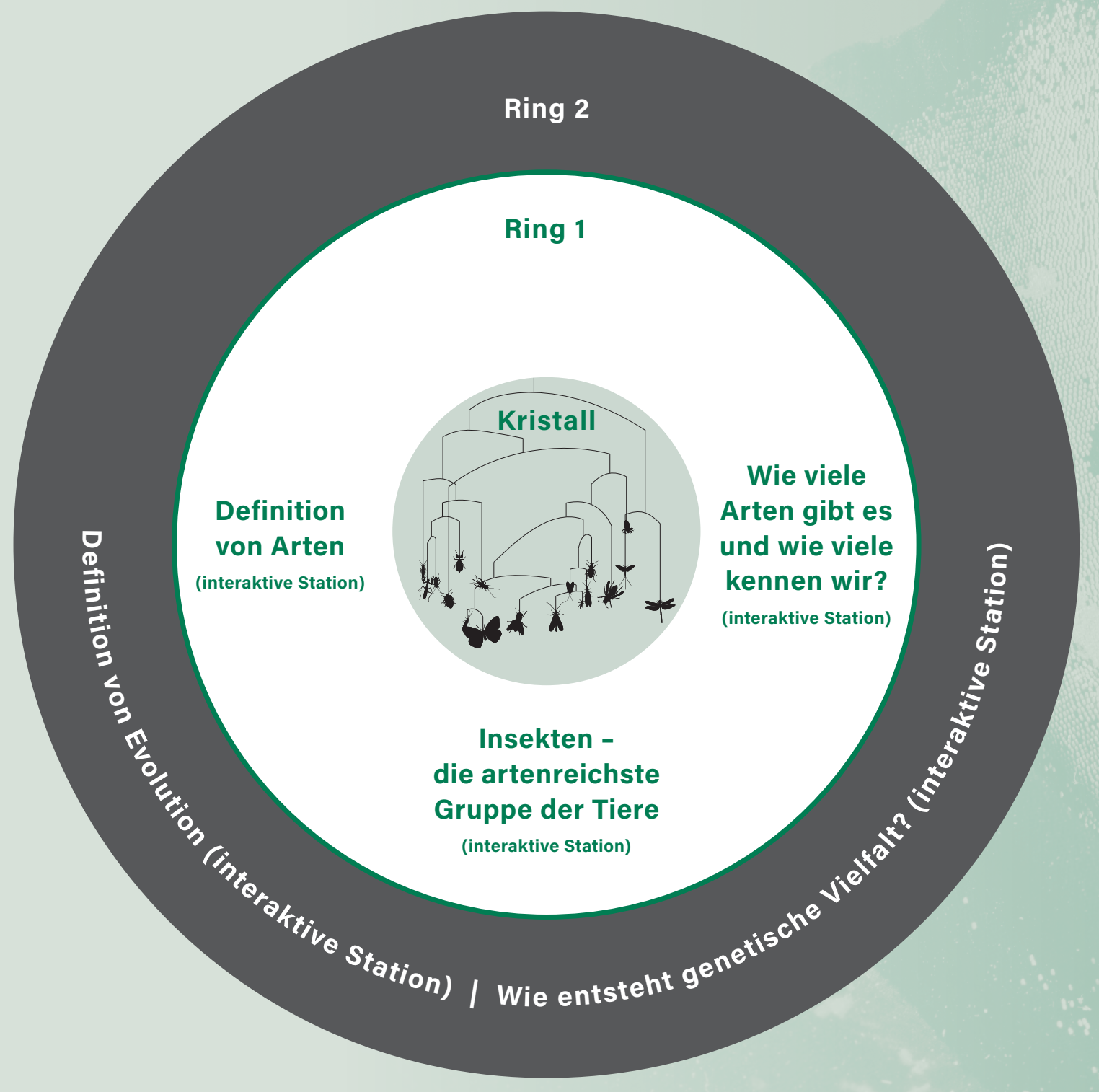

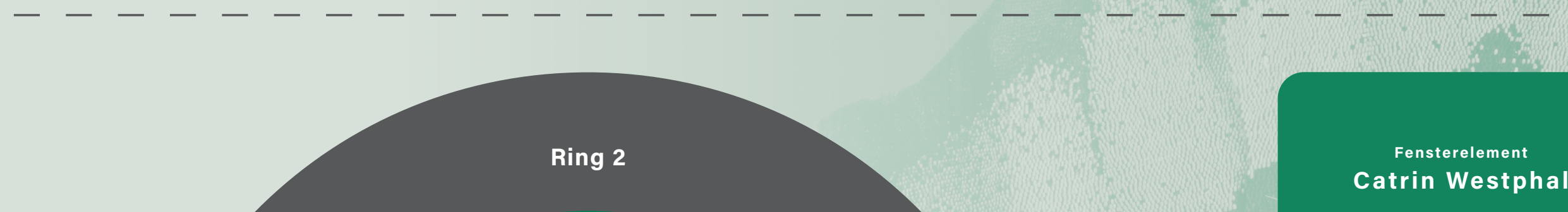

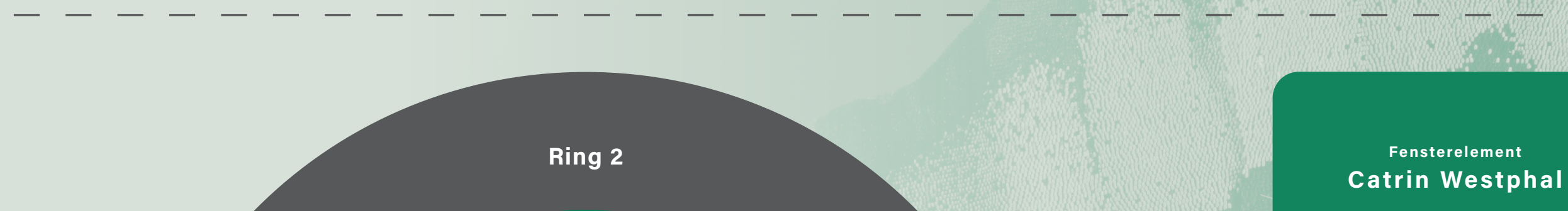

Konnektor:

Fledermaus

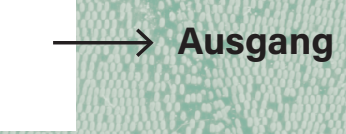




\section{Der Konnektor}

Natürlich sind Insekten nur ein Teil der Geschichte und Nahrungsgrundlage für viele andere Tiere.

Am Ende des Raumes stoßen die Besucher auf eine Motte, die der Flechtenmotte ähnelt, die sie dorthin geführt hat. Diese Motte wird von einer Fledermaus gefressen, die zusammen mit den neugierigen Besucher*innen ihren Weg in den nächsten Raum antritt.

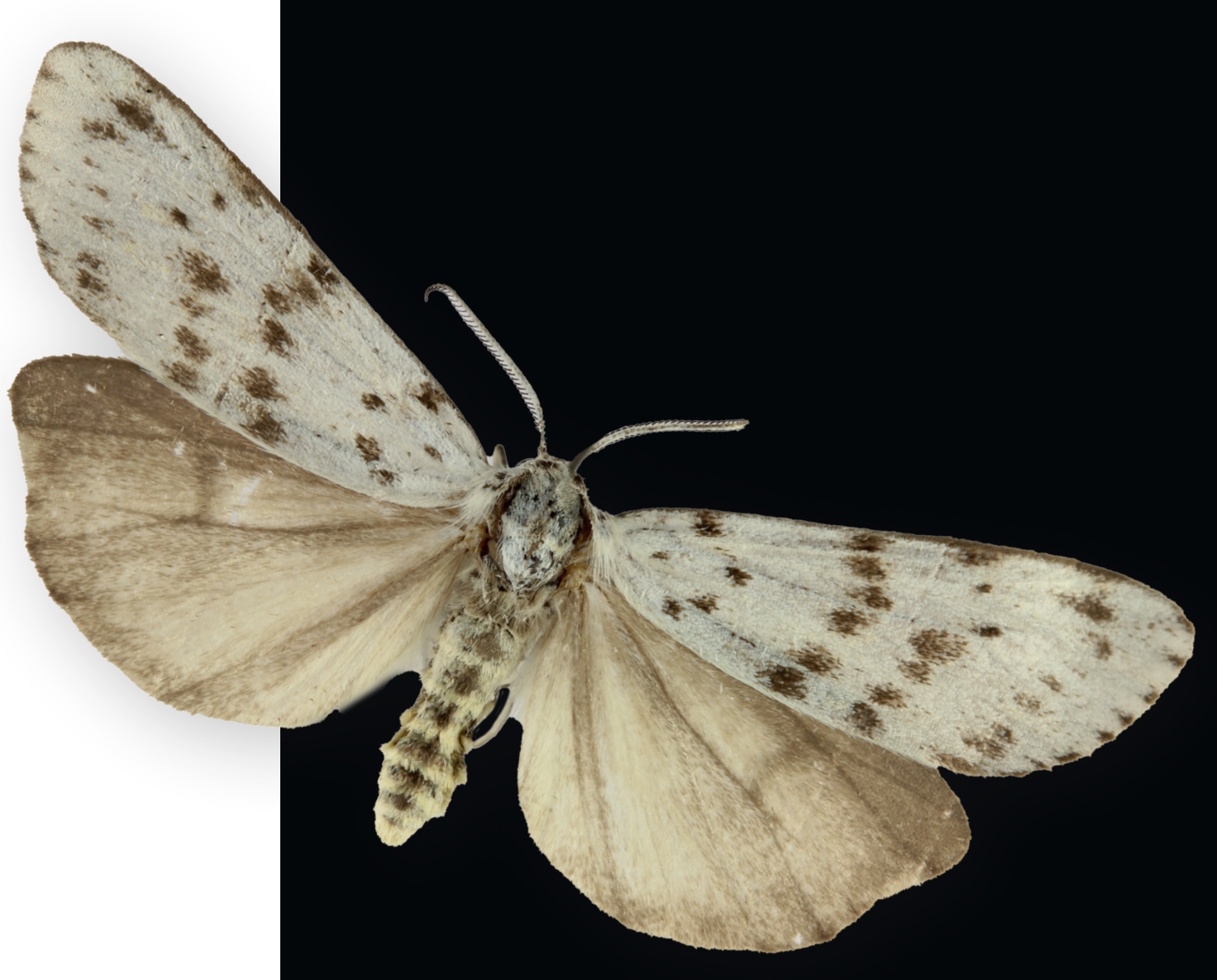




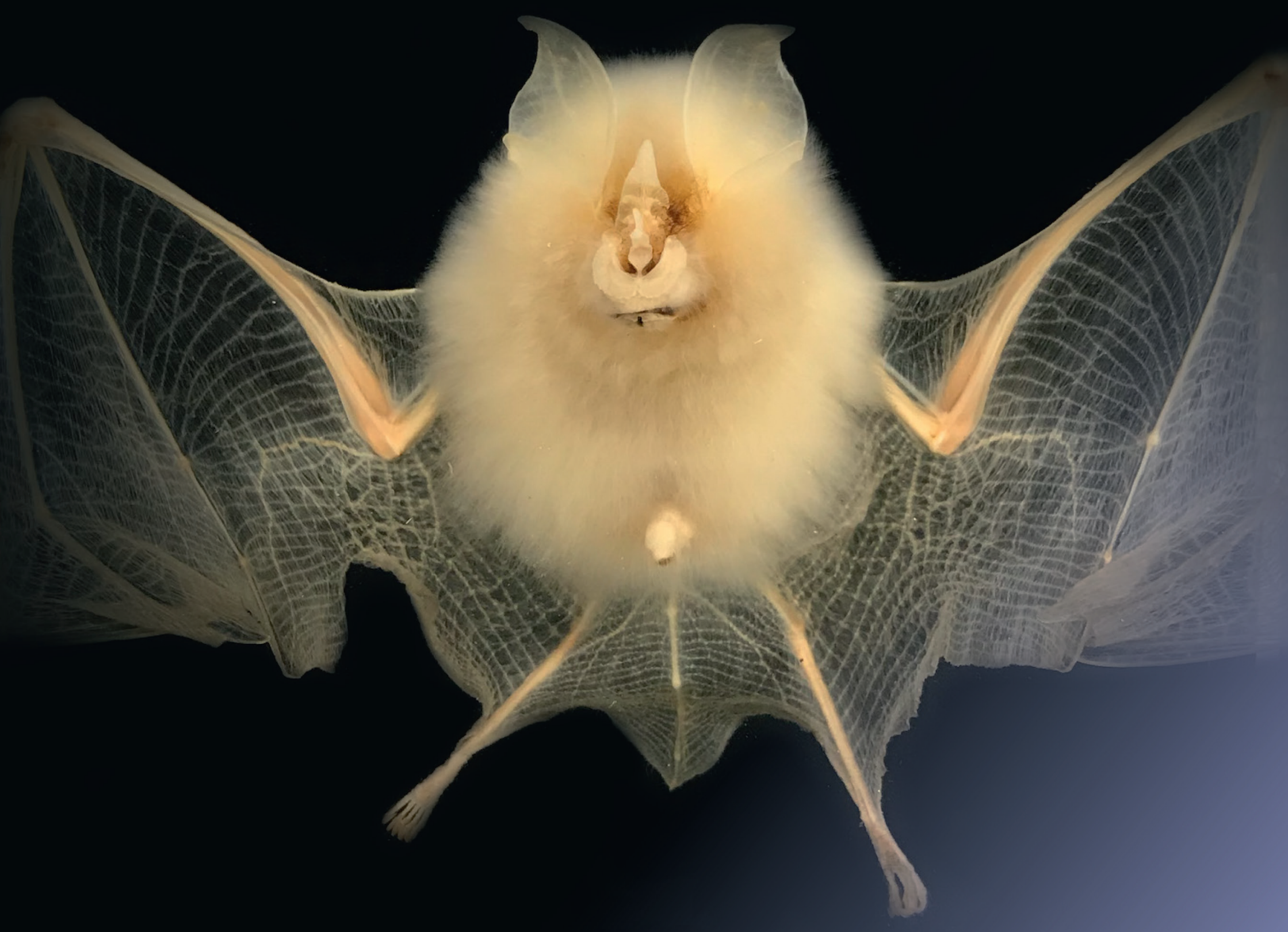




\section{Gang durch die Ausstellung}

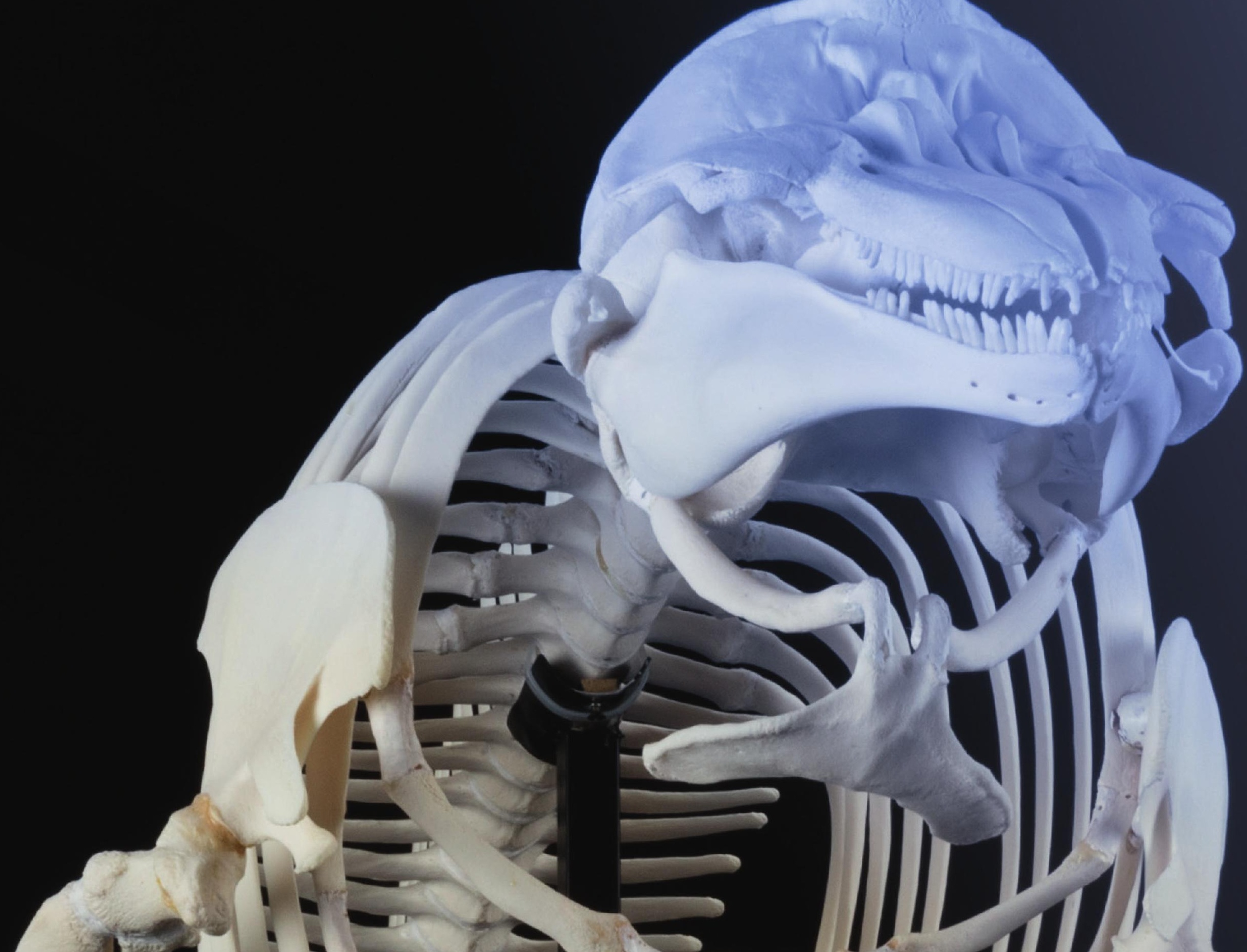




\section{Was ist Evolution?}

"So geht aus dem Kampfe der Natur, aus Hunger und Tod unmittelbar die Lösung des höchsten Problems hervor, das wir zu fassen vermögen, die Erzeugung immer höherer und vollkommenerer Thiere. Es ist wahrlich eine grossartige Ansicht, dass der Schöpfer den Keim alles Lebens, das uns umgibt, nur wenigen oder nur einer einzigen Form eingehaucht hat, und das, während unser Planet den strengsten Gesetzen der Schwerkraft folgend sich im Kreise geschwungen, aus so einfachem Anfange sich eine endlose Reihe der schönsten und wundervollsten Formen entwickelt hat und noch immer entwickelt.»

- Charles Darwin 


\section{Szenografie \& Motive}

Wenn die Besucher*innen das dritte Kapitel betreten, erkennen sie, dass die Fledermaus sie in die Welt der Säugetiere gebracht hat. Sie befinden sich in einem atmosphärischen Raum, dessen Szenografie einen Übergang vom Land, an der Seite des Eingangs, zum Meeresgrund, am anderen Ende, rekonstruiert.

Der Kristall besteht aus einer Installation dreier Skelette: einem Nilpferd, einem Belugawal und einem Fossil (z. B. Pakicetus), welches eine nah verwandte, ausgestorbene Art der Wale repräsentiert. Das Fossil hilft, den landlebenden Vorfahren der heutigen Wale zu rekonstruieren. Den Besucher*innen wird schnell vermittelt, dass sich in diesem Raum alles um Säugetiere und ihre Evolution dreht. Der Kristall erzählt von der Lösung eines der Rätsel, die Evolutionsbiologen bereits lösen konnten: Die heutigen aquatischen Wale und die terrestrischen Flusspferde haben einen gemeinsamen Vorfahren. Ein prächtiges 17 $\mathrm{m}$ langes Skelett eines Pottwals aus der Zoologischen Sammlung, das in einem anderen Teil des Gebäudes gezeigt wird, wird ebenfalls in Kontext gesetzt. Rund um den Kristall gibt es verschiedene Interaktionen, die erklären, wie Evolution, neben anderen Mechanismen, durch das Wechselspiel von natürlicher Selektion und Aussterben angetrieben wird.
Der Kristall: Schlüsselexemplare

- Exemplare, die die Evolution der

Säugetiere verdeutlichen

- Skelette und Dermoplastiken: Säugetiergruppen und Evolution: Monotremata (Schnabeltier, Schnabeligel), Beuteltiere (Känguru, Wallaby, Wombat etc.) und Plazentatiere (Fledermäuse, Primaten etc.)

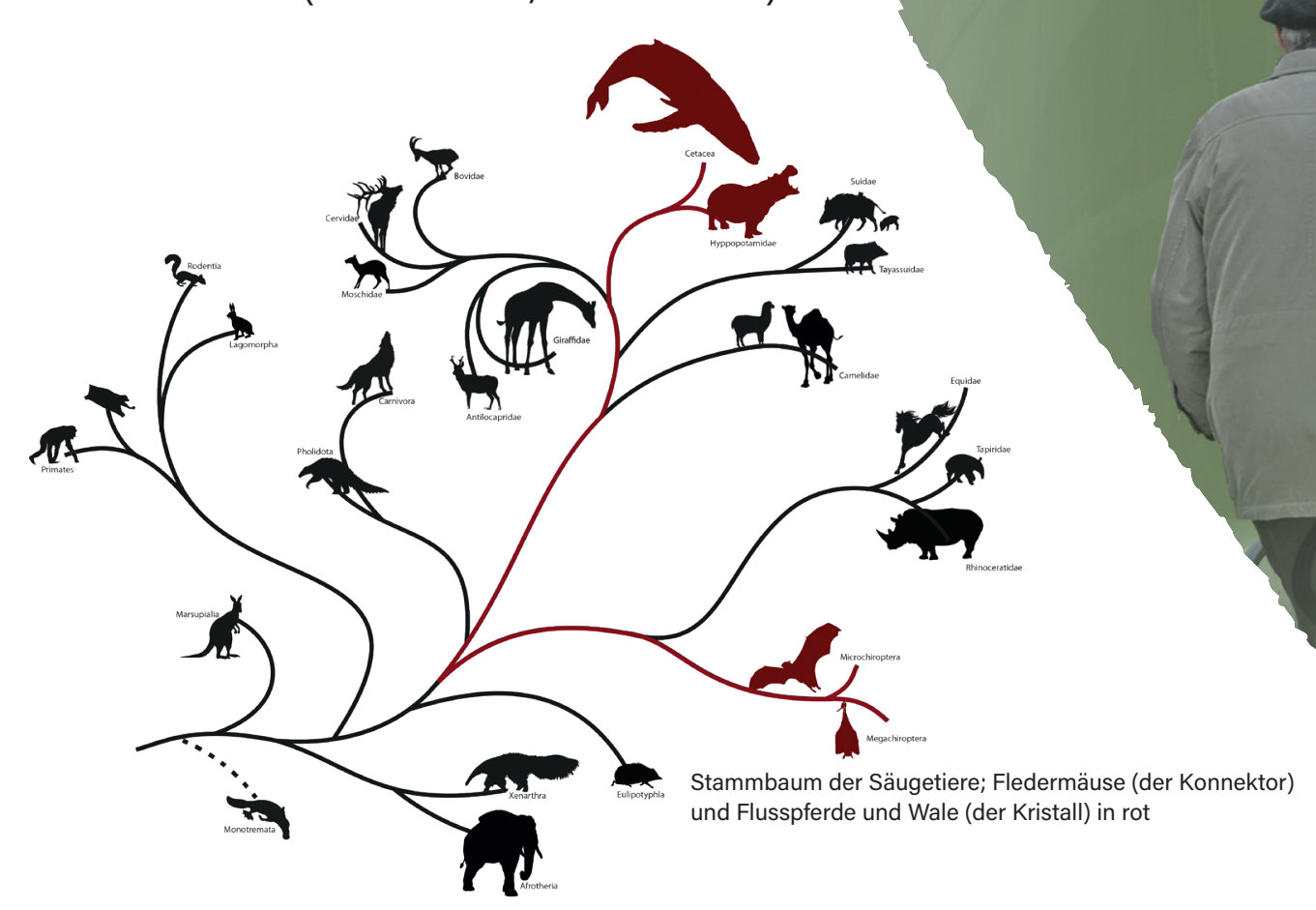




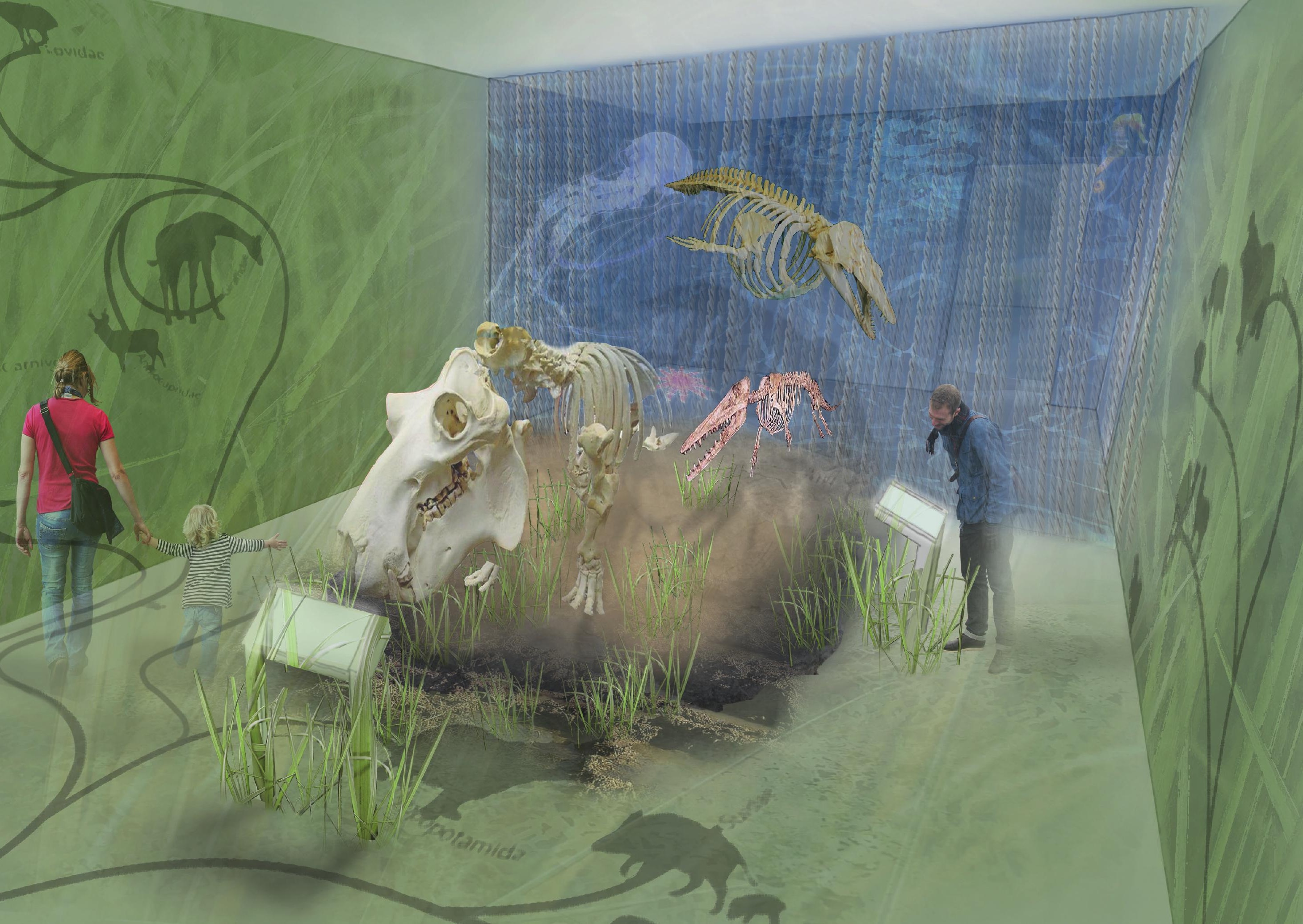




\section{Themen \& Interaktionen}

Ring 1: interaktive Stationen

- Systematik II: Phylogenetik; der Baum des Lebens (z. B. Evolution der Säugetiere)

- Evolution von terrestrischen zu marinen

Ökosystemen (bei Säugetieren, z. B. Evolution der Wale)

- Wal-Kadaver als Ökosystem

\section{Tiny Explorers}

Kinder werden aufgefordert, sich auf den Bauch zu legen und entweder ihre Beine seitwärts wie ein Fisch, oder auf und $a b$, wie ein Wal zu bewegen. Sie werden feststellen, dass sie wie Wale sind - oder vielmehr, dass Wale nicht wie Fische gebaut sind.

\section{Ring 2: interaktive Stationen}

- Diversifikation vs. Aussterbeereignisse (Diversifikationsereignisse, Aussterbeereignisse über erdgeschichtliche Zeiträume und Massenaussterben)

- Stehen wir am Rande des 6. Massenaussterbens? Das Anthropozän

- Verschiedene Skelett-Rätsel: Besucher*innen können anhand des Schädels erraten, zu welcher Tierart dieser gehört. Besucher*innen können Teile eines Skeletts zusammensetzen, einen fehlenden oder einen „falschen" Knochen finden.

Ring 3: Fensterelemente

- Wie die Wissenschaft herausfand, dass Nilpferde und Wale eng miteinander verwandt sind

- Die Bedeutung von Darwins Theorie

- Sand und interstitielle Fauna: Die „unbekannten“ Phyla: Loricifera und Gnathostomulida

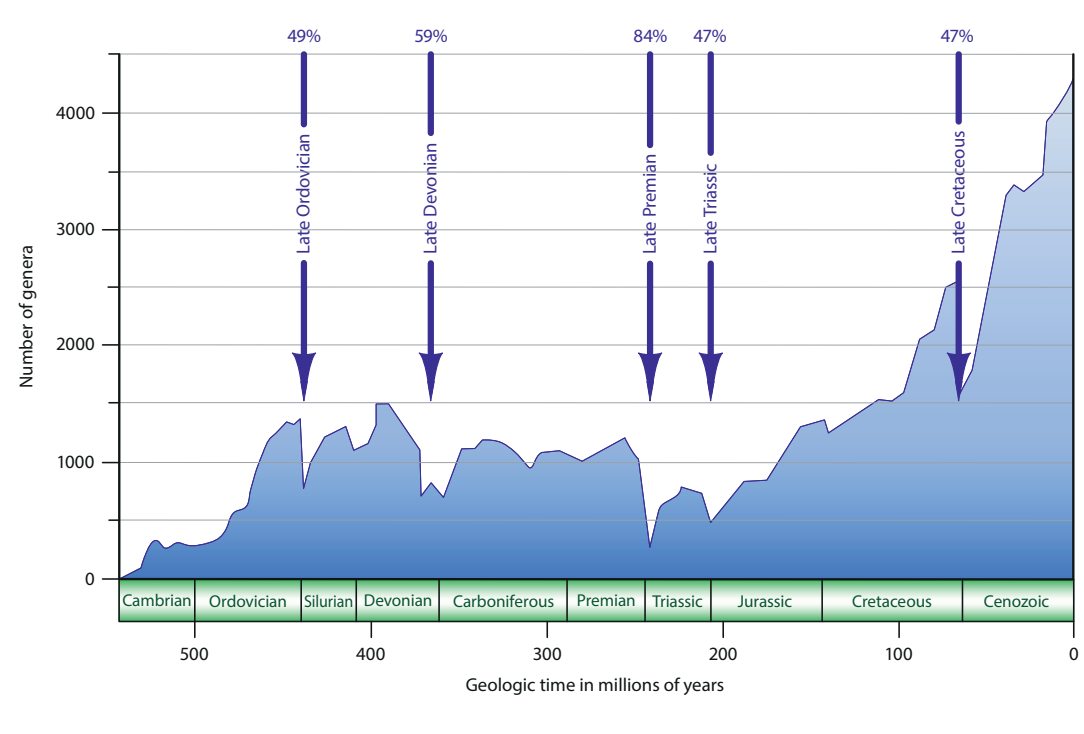

Die fünf großen Massenaussterben seit dem Kambrium. Der angenommene Prozentsatz ausgestorbener Gattungen ist angegeben (Abbildung modifiziert nach Reitner, 2011).
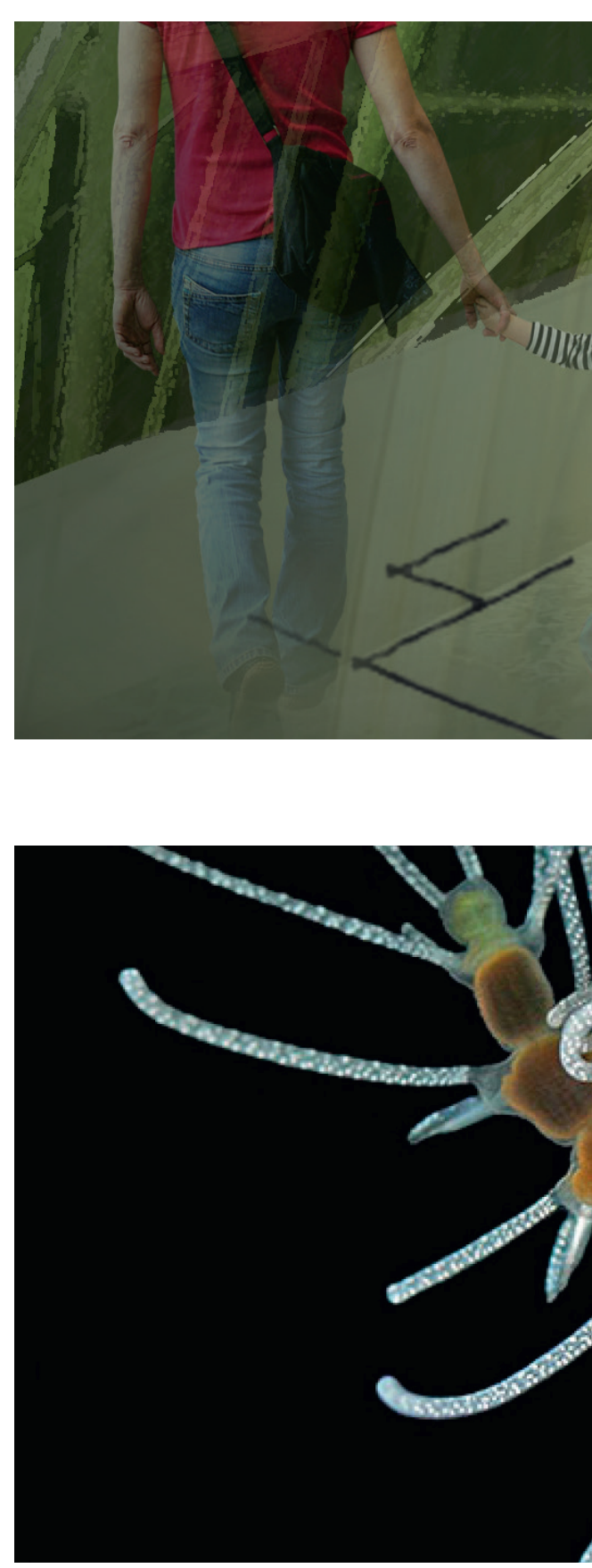


\section{Räumliche Aufteillung}

Prof. Dr. Christoph Bleidorn

Seit 2017 Professor und Leiter der Abteilung für Evolution und Biodiversität der Tiere, J.F. Blumenbach Institut, und seit 2018 Direktor des Zoologischen Museums der Universität. Seine Forschungsinteressen umfassen die Phylogenie und Evolution von Anneliden, die Evolution symbiotischer Beziehungen zwischen Insekten und Bakterien sowie die Bestandsaufnahme der lokalen Bienenfauna.

\section{Eingang}

Fensterelement

\section{Dr. Maria Teresa Aguado Molina}

Kuratorin der Zoologischen Sammlung und des Biodiversitätsmuseums und seit 2019 Dozentin am J.F. Blumenbach Institut. Ihre Forschungsinteressen umfassen die Biodiversität der marinen Wirbellosen, ihre Evolution, die phylogenetischen Beziehungen, Entwicklungsmuster und deren Anpassungen an extreme Lebensräume, wie die Tiefsee.

\section{Prof. Dr. Julia Fischer}

Julia Fischer ist Professorin für Primatenkognition der Universität Göttingen und seit 2016 Leiterin der Kognitiven Ethologie des Deutschen Primatenzentrums in Göttingen. Zu den Schwerpunkten ihrer Forschung gehören Primatenkognition, Kommunikation und Sozialverhalten sowie die Evolution von Sprache.

\section{Christoph Bleidorn}


Ring 3:

Vorhang / Raumtrennung zwischen terrestrischen und marinen Ökosystem

- - - - - - - - - - - - - - - -

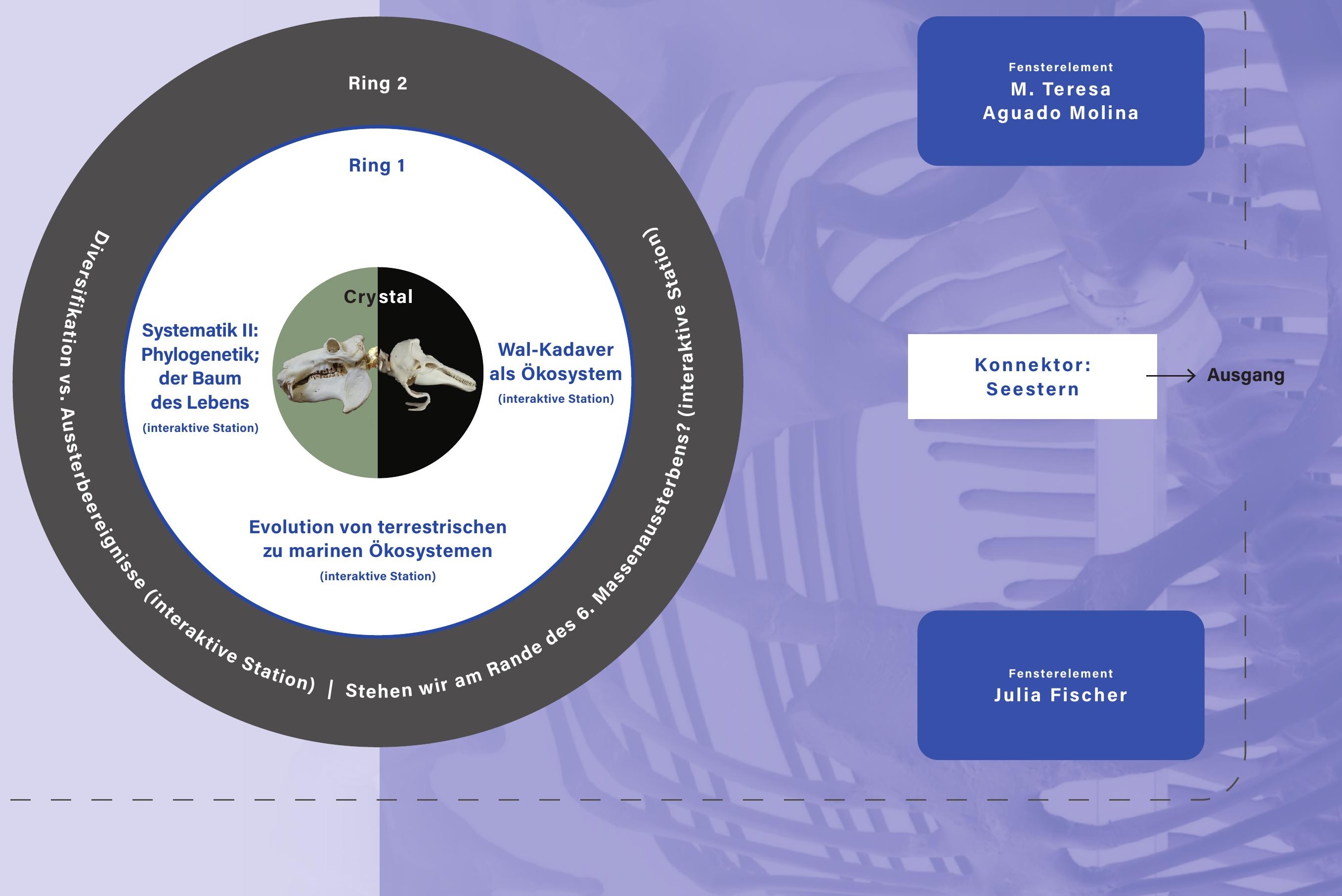




\section{Der Konnektor}

Wenn die Besucher*innen den Raum durchquert haben und an dem Kristall vorbeigegangen sind, befinden sie sich auf dem Meeresgrund.

Illustriert wird dieser durch die Projektion eines Wal-Kadavers, der von verschiedenen Organismen, darunter auch Seesterne, gefressen wird. Einer der Seesterne krabbelt über den Boden, stirbt und wird im nächsten Raum an das Ufer gespült.

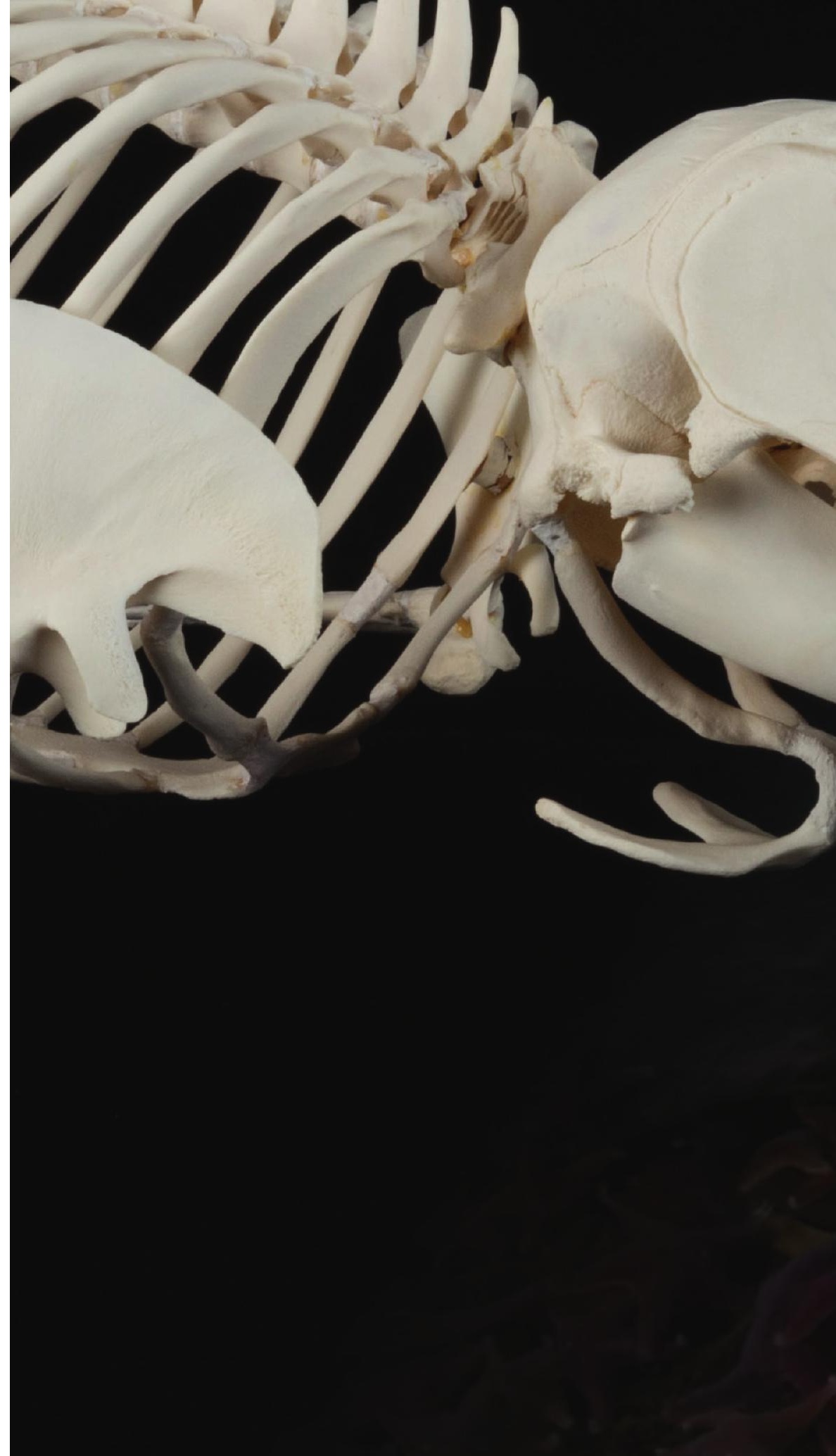




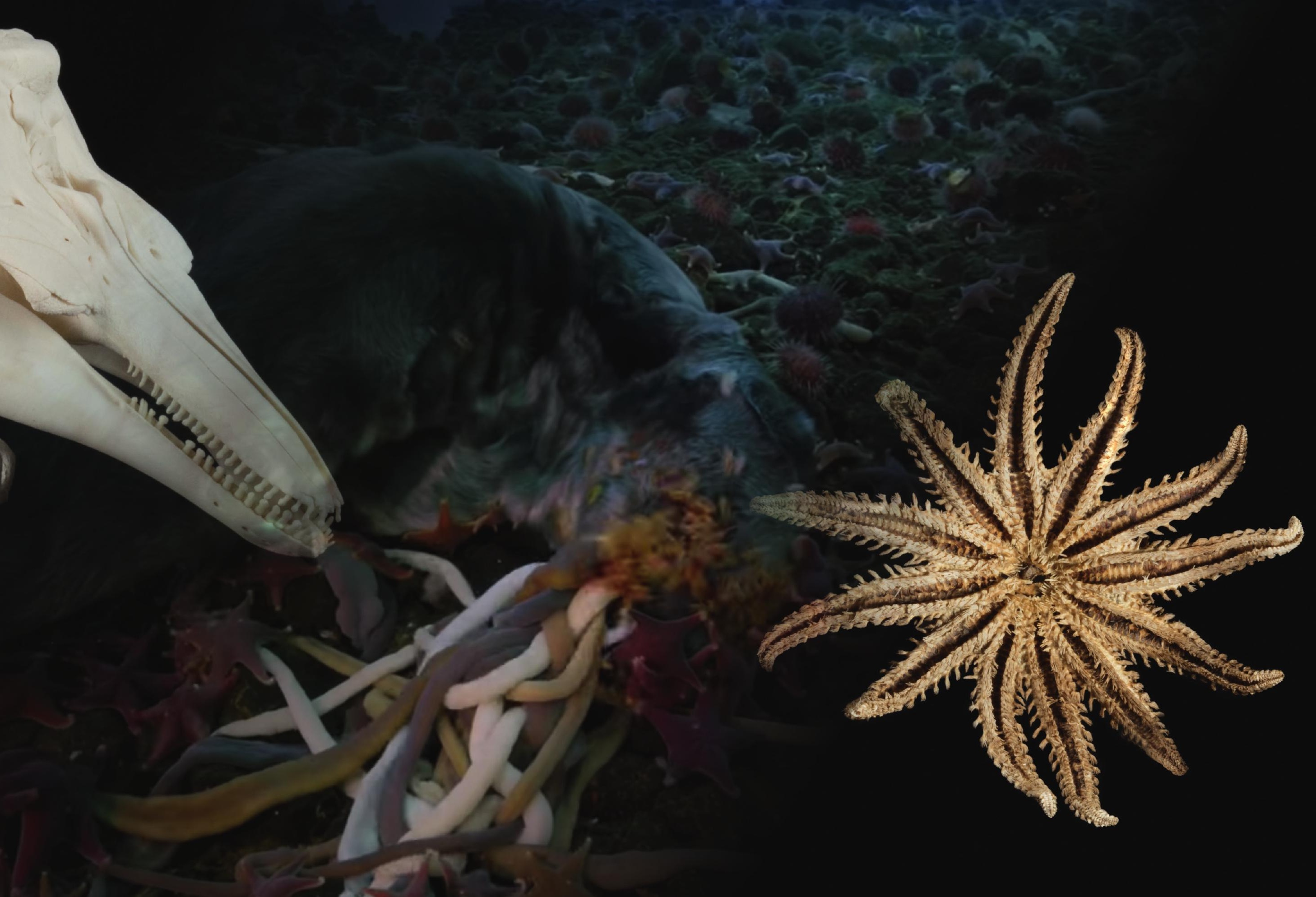




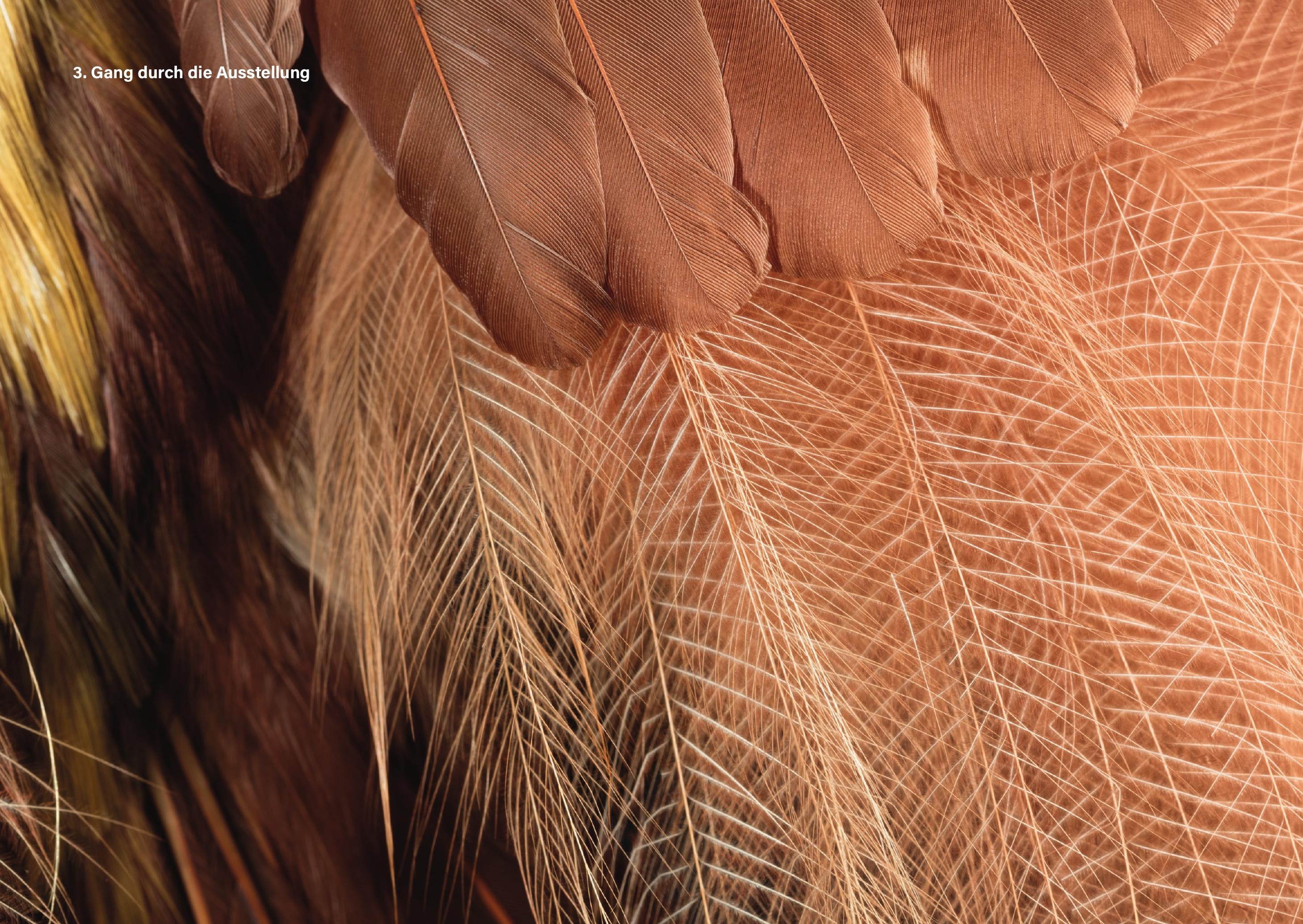




\section{Warum ist}

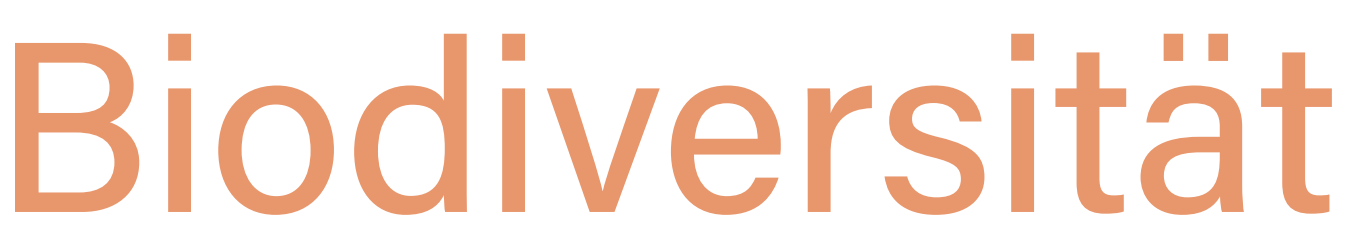

wichtig?

«Biologische Vielfalt [...] ist der Schlüssel zur Erhaltung der Welt, wie wir sie kennen. Das Leben an einem Ort, der von einem vorbeiziehenden Sturm heimgesucht wird, kommt schnell zurück, weil noch genügend Vielfalt vorhanden ist.»

- Edward O. Wilson 


\section{Szenografie \& Motive}

Nachdem sie mit den Grundlagen der Evolutionsbiologie vertraut gemacht wurden, gelangen die Besucher*innen mitten in eine komplexe Umwelt.

Kapitel 4 spielt in einem Dschungel einer indonesischen Insel, an deren Küste unser Seestern gestrandet ist. Der Dschungel ist voller verschiedener Formen, Gerüche und Geräusche; vor allem Pflanzen spielen eine große Rolle in diesem Raum. Die beiden Kernthemen in diesem Kapitel sind Resilienz und Fragilität. Die Besucher*innen lernen, dass Ökosysteme mit hoher Artenvielfalt widerstandsfähiger sind als Ökosysteme mit einer geringen Anzahl von Arten. Jedoch hat jedes Ökosystem auch einen gewissen Grad an Fragilität. Die Einführung eines einzigen neuen Raubtiers auf einer Insel kann beispielsweise das komplette System verändern und zum Aussterben ganzer Arten führen.

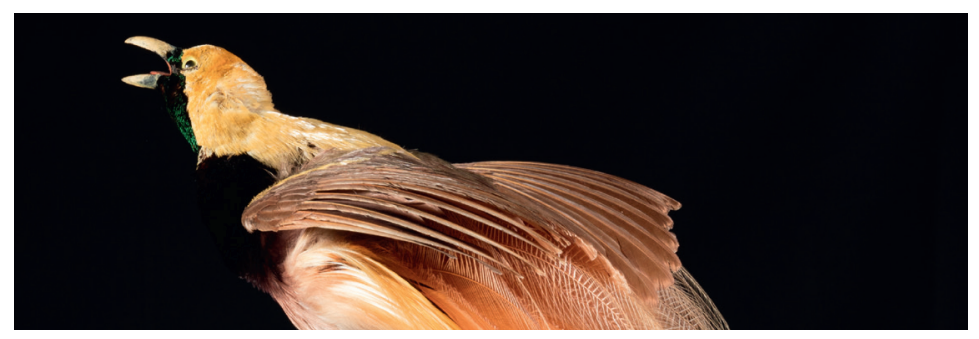

Paradisaea minor Shaw, 1809 Zoologische Sammlung / Universität Göttingen 78
Der Kristall: Schlüsselexemplare Der tropische Regenwald in Indonesien

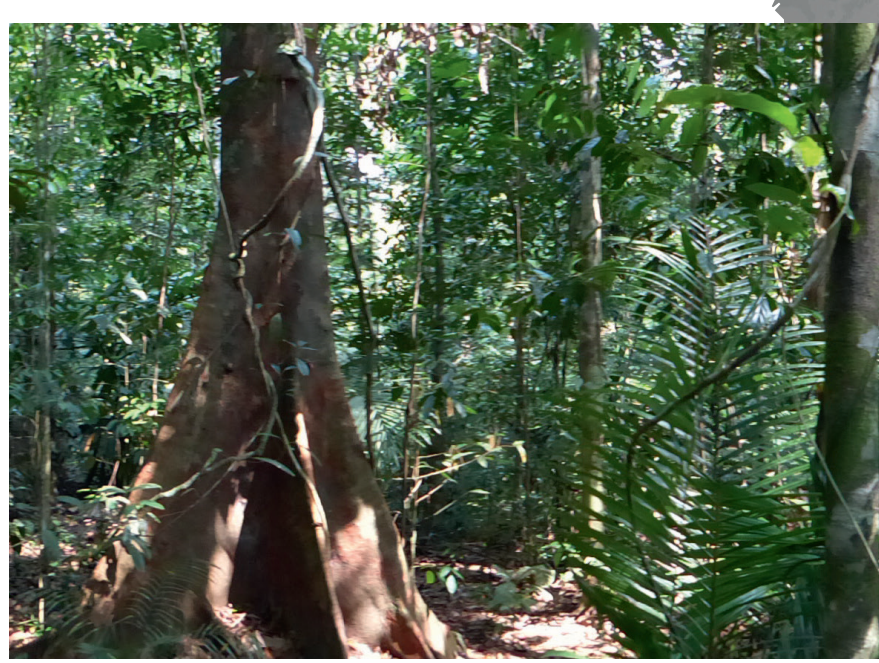

Der Kristall: Der indonesische Dschungel. Jambi, Sumatra (Foto K. Rembold)

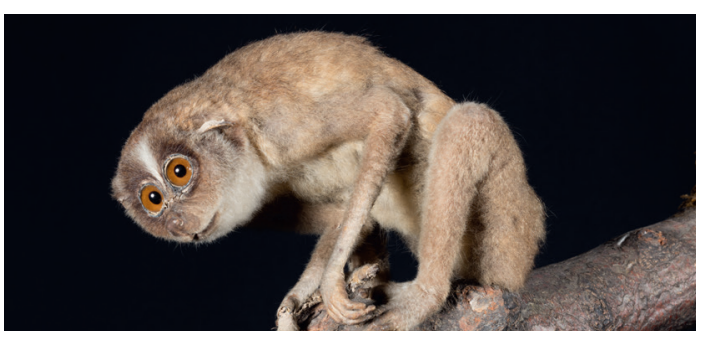

Loris tardigradus (Linnaeus, 1758) Zoologische Sammlung / Universität Göttingen

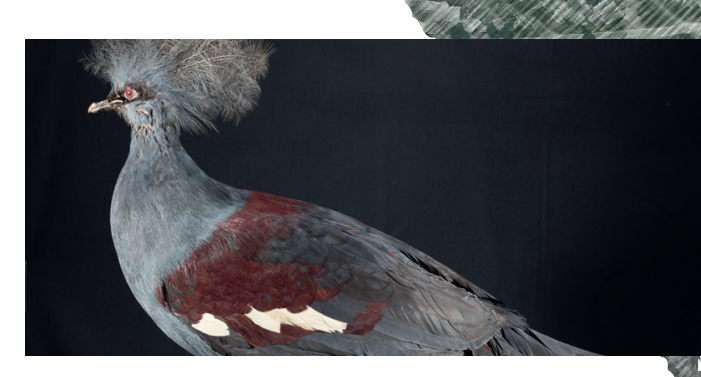

Goura cristata (Pallas, 1764) Zoologische Sammlung / Universität Göttingen 


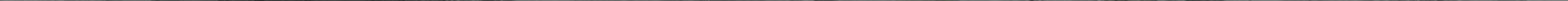




\section{Themen \& Interaktionen}

Ring 1: interaktive Stationen

- Biodiversitäts-Hotspots

- Biodiversität und Ökosystemfunktionen

(Verbindung zu „warum wollen

wir die Biodiversität erhalten?")

- Rückkopplungsschleifen

(z. B. die voneinander abhängigen

Populationen eines Räubers

und seiner Beute)

\section{Ring 2: interaktive Stationen}

- Endemismus und Artenreichtum auf Inseln (globale Vielfalt der Inselflora)

- Resilienz in Ökosystemen und Fragilität von Insel-Ökosystemen

- Das Orchester der Artenvielfalt: Besucher*innen können einem Orchester beim Spielen zuhören. Anschließend können sie Instrumente einzeln "löschen". Wie verändert sich die Musik? Wann wird sie unerkennbar?

- Eine Simulation, bei der die Besucher Ökosysteme mit vielen oder wenigen Arten aufbauen und dann sehen, was im Falle einer Störung oder Veränderung in der Umgebung passiert.
Ring 3: Fensterelemente

- Humboldt (der auch an der Georg-AugustUniversität studierte) und seine Ideen

- Insel-Biogeographie (z. B. Art-HabitatBeziehungen, Wilson, Mc Arthur und Simberloff)

Tiny Explorers

- Ein „Bandolino-Puzzle", bei dem Kinder Vogelschnäbel und Nahrung oder Insektenrüssel und verschiedene Blumen zusammenbringen können.

- Ein „Wimmelbild”, das ausgemalt werden kann.

- Eine haptische Station, an der verschiedene Arten von Blättern oder Rinde erfühlt werden können.

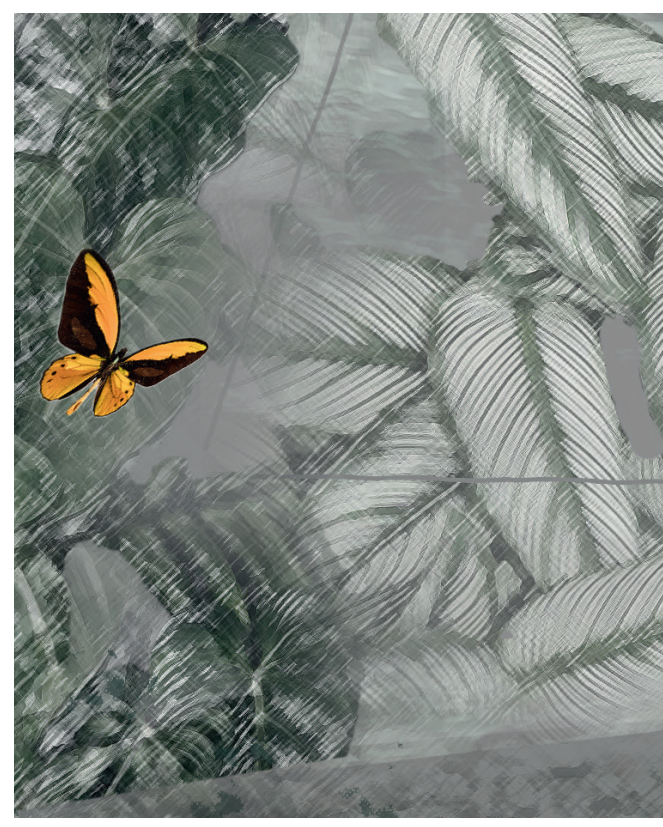




\section{Räumliche Aufteillung}

Prof. Dr. Holger Kreft

Holger Kreft ist seit 2015 Leiter der Abteilung Biodiversität, Makroökologie und Biogeographie und Professor an der Fakultät für Forstwissenschaften und Waldökologie. Er untersucht ökologische und biogeografische Muster um die Entwicklungen räumlicher Verteilung von Biodiversität zu dokumentieren und verstehen.

Prof: Drr Hermann Behling

Hermann Behling ist seit Oktober 2005 Professor für Botanik und Leiter der Abteilung Palynologie und Klimadynamik am Albrecht-von-Haller-Institut für Pflanzenwissenschaften. Seine Forschungsschwerpunkte liegen in terrestrischer und mariner Palynologie, Palaeoökologie, Biodiversitätsdynamik, Palaeoklimatologie, Bestäubung, Feuer- und Siedlungsgeschichte.

\section{Eingang}

Fensterelemente Holger Kreft 
Ring 3

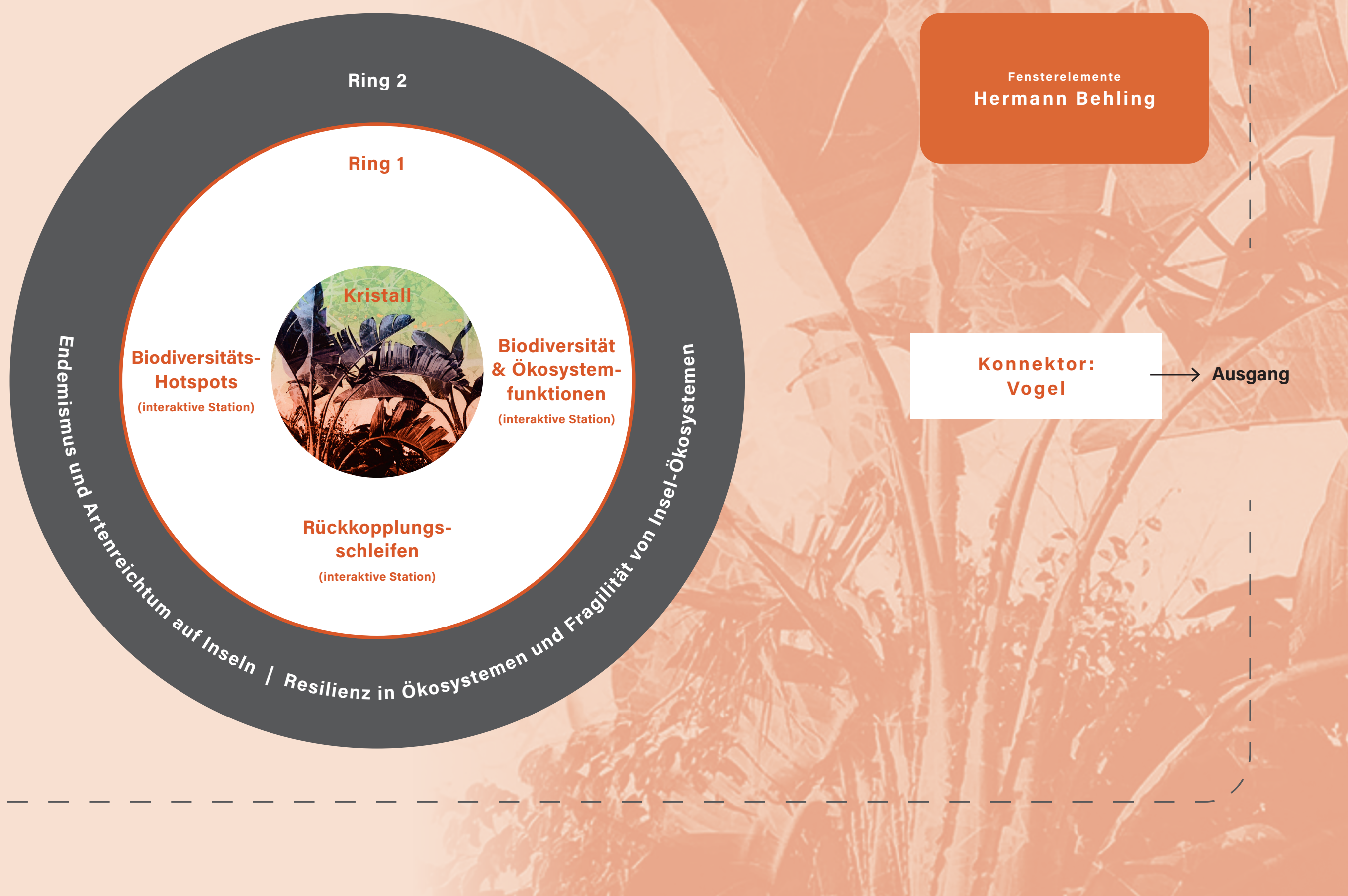




\section{Der Konnektor}

In Indonesien werden riesige Flächen des tropischen Regenwaldes brandgerodet, um Platz für PalmölPlantagen zu schaffen.

Der Konnektor zum nächsten Raum ist ein tropischer Vogel, welcher im Regenwald lebt. Er fliegt durch einen (projizierten) Vorhang aus Feuer. Das Feuer ist eine schnelle und günstige Methode, das Ökosystem des Regenwaldes in ein für Monokulturen nutzbares Gebiet zu verwandeln, was, wie man sich vorstellen kann, verheerende Folgen hat. Die Besucher*innen "gehen durch das Feuer", um in den nächsten Raum zu gelangen. Der Vogel ist zwar auf der anderen Seite noch am Leben, aber was ist mit all den anderen Tieren des Regenwaldes passiert? 


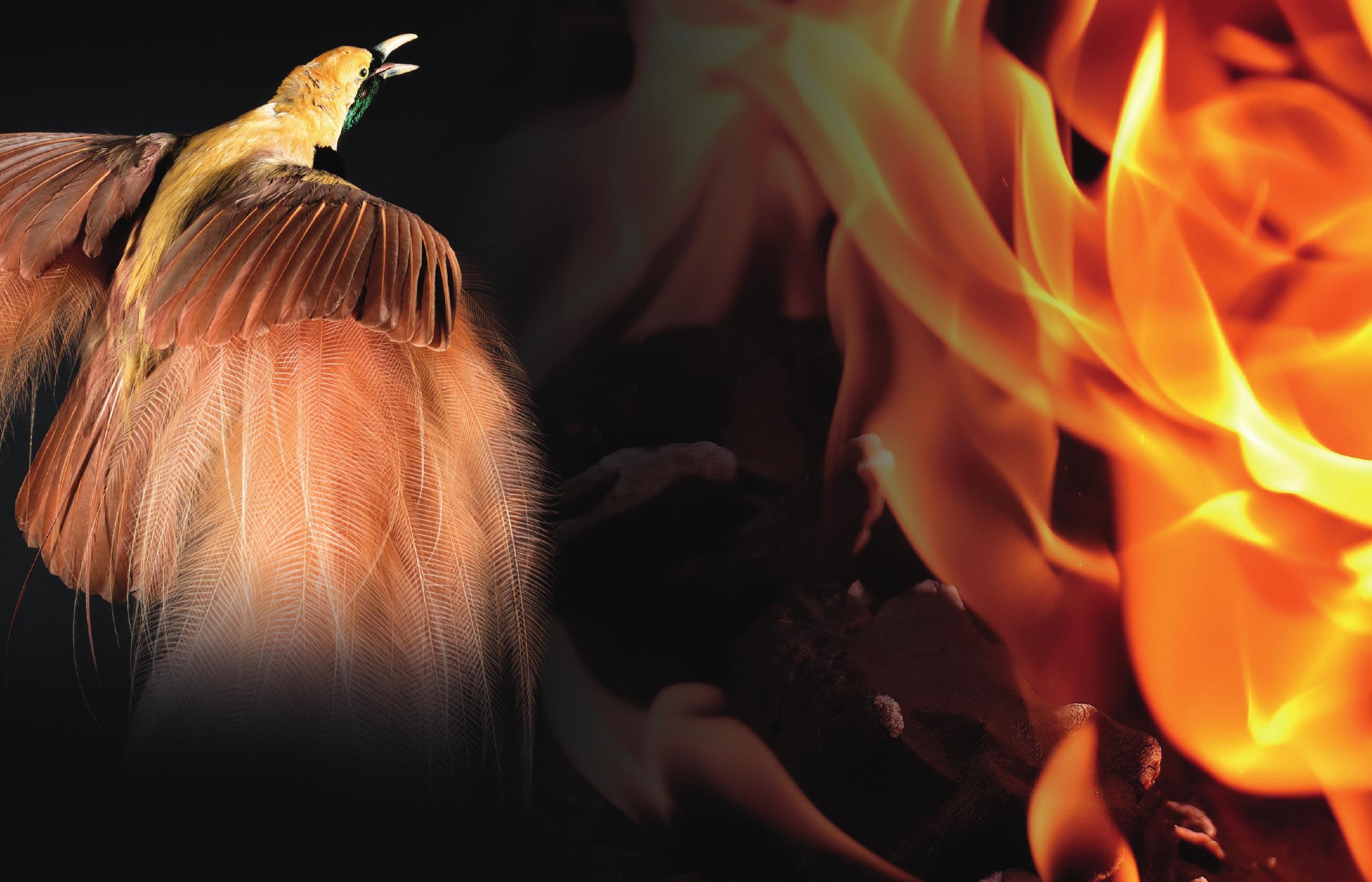




\section{Gang durch die Ausstellung}
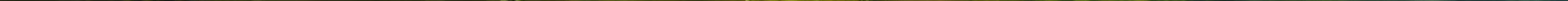


\section{Menschlicher}

Einfluss

«Das Holozän ist vorbei. Der Garten Eden existiert nicht mehr. Wir haben die Welt so stark verändert, dass Wissenschaftler sagen, dass wir in einem neuen geologischen Zeitalter leben: das Anthropozän, das Zeitalter der Menschen.»

- David Attenborough

\section{Kapitel 5}




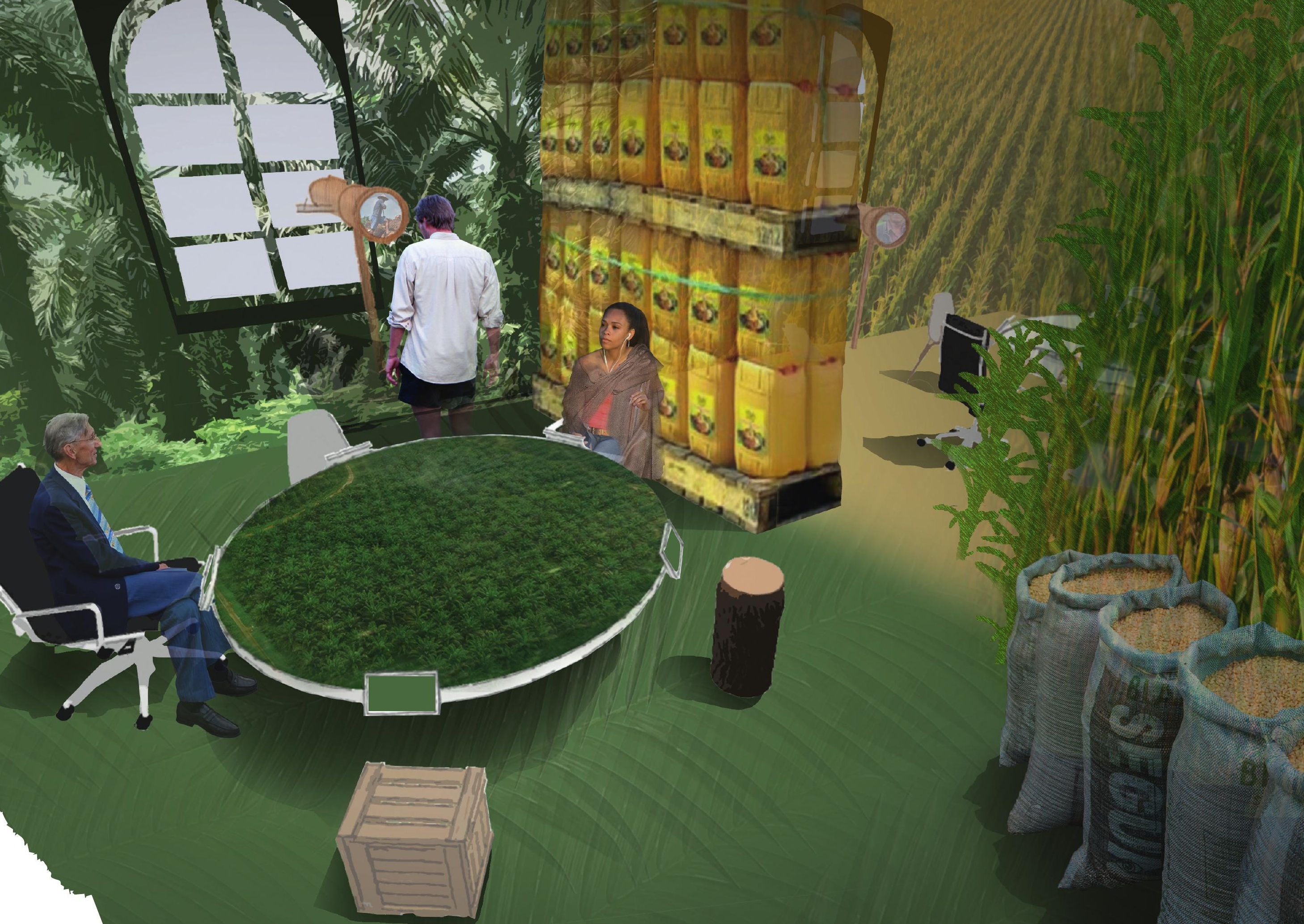




\section{Themen \& Interaktionen}

\section{Ring 1: interaktive Stationen}

Indonesien - fünf verschiedene Protagonist*innen (Videos): Ein Tisch mit einem Modell (Drohnenansicht) einer Palmölplantage in der Mitte. Die Besucher*innen können sich an den Tisch setzen und "treffen" die Protagonist*innen, die darüber sprechen, was die Plantage für ihre Arbeit oder ihr Leben bedeutet. Das kann ein Bauer oder eine Bäuerin sein oder Arbeiter*innen, die von dem Palmöl profitieren, Umweltaktivist*innen, die gegen den Verlust des Regenwaldes kämpfen, Wissenschaftler*innen oder Lokalpolitiker*innen. Die unterschiedlichen Ansichten und Konflikte werden diskutiert und mögliche Lösungen vorgeschlagen.

Deutschland - Rollenspiel: Ein Tisch mit dem Modell eines Maisfeldes in der Mitte. Die Besucher*innen können sich hinsetzen und spielen ein Rollenspiel. Die Charaktere sind ähnlich denen des anderen Tisches (Bauer*innen, Aktivist*innen, Wissenschaftler*innen, Konsument*innen ...), jedoch ist der Schauplatz in Deutschland. Beim Spielen werden die Besucher*innen feststellen, dass die Konflikte ähnlich sind und kreative Lösungsansätze gefragt sind.

\section{Tiny Explorers}

Eine Multimedia-Station, bei der die Besucher*innen ein
Gebiet mit vielen kleinen oder wenigen großen Lebensräumen erschaffen können. Das Ergebnis zeigt, wie viele Arten angezogen werden.

\section{Ring 2: interaktive Stationen}

\section{Indonesien:}

- Eine Multimedia-Station, die zeigt, wie sich Individuenreichtum und Artenvielfalt in den verschiedenen Umgebungen verändern

- Animation Farbmetapher

\section{Deutschland:}

- Globale Folgen des menschlichen Einflusses auf den Verlust der Biodiversität (z. B. menschlicher Lebensstil in westlichen Gesellschaften)

- Insektensterben (Verlust der Biodiversität verbunden mit dem Verlust von Lebensräumen)

\section{Ring 3: Fensterelemente}

- Wie sich Artenreichtum und Artenvielfalt mit der Umwandlung des Ökosystems in eine Monokultur verändern.

- Öffnen Sie das Fenster und besuchen Sie eine Ölpalmenplantage.

- Öffnen Sie das Fenster und besuchen Sie ein Maisfeld.

- Audioaufnahme - Vermessung der Biodiversität

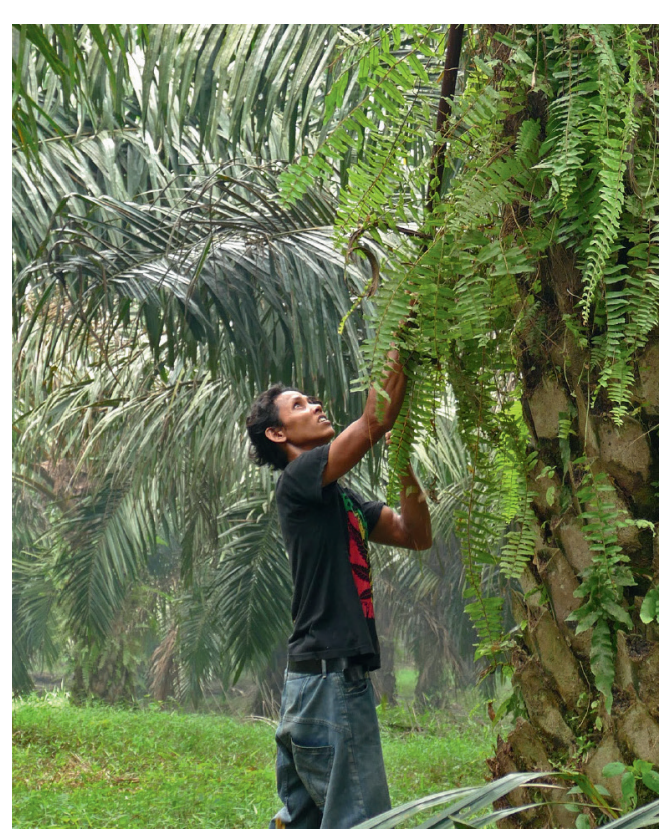

Ernte von Ölpalmen,

Jambi, Indonesien (Foto von S. Eckelmann)

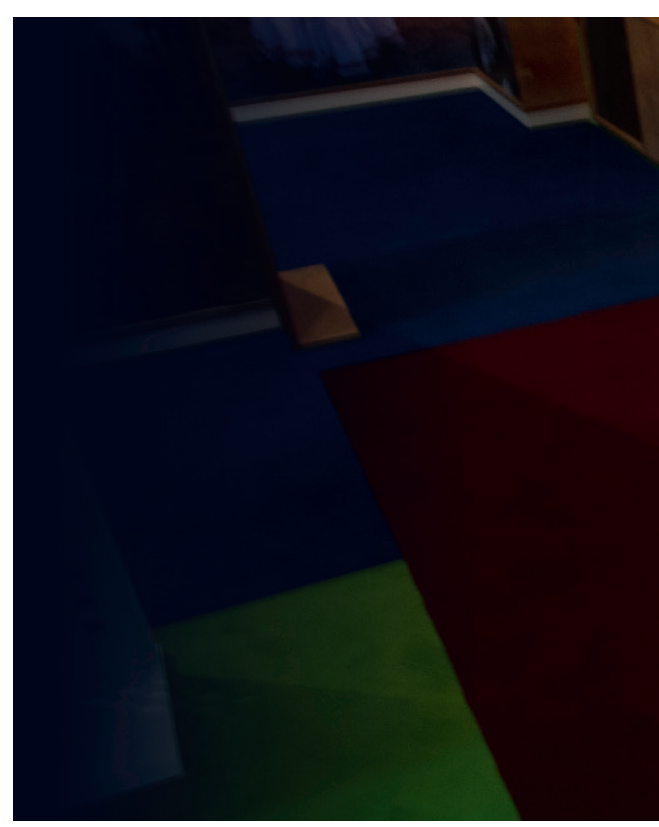




\section{Räumliche Aufteilung}

Prof. Dr. Matin Qaim

Matin Qaim ist Professor für Welternährungswirtschaft und Rurale Entwicklung am Department für Agrarökonomie und Rurale Entwicklung. Diese Position hat er seit 2007 inne. Zu seinen Forschungsgebieten zählen Ernährungssicherung und nachhaltige Ernährungssysteme, nachhaltige Landnutzung und Auswirkungen von technischen und institutionellen Innovationen im Kleinbauernsektor.

\section{Prof: Dr. Stefan Scheu}

Stefan Scheu ist seit 2008 Professor und Leiter der Abteilung für Tierökologie am J. F. Blumenbach Institut. Seine wissenschaftliche Arbeit konzentriert sich unter anderem auf die Struktur und Funktionsweise von Bodentiergemeinschaften und die Wechselbeziehungen zwischen dem unter- und oberirdischen System. 


\section{Ring 3}

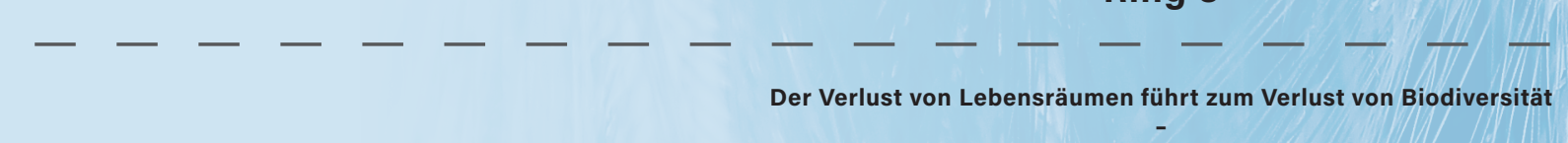
Auswirkungen des Menschen

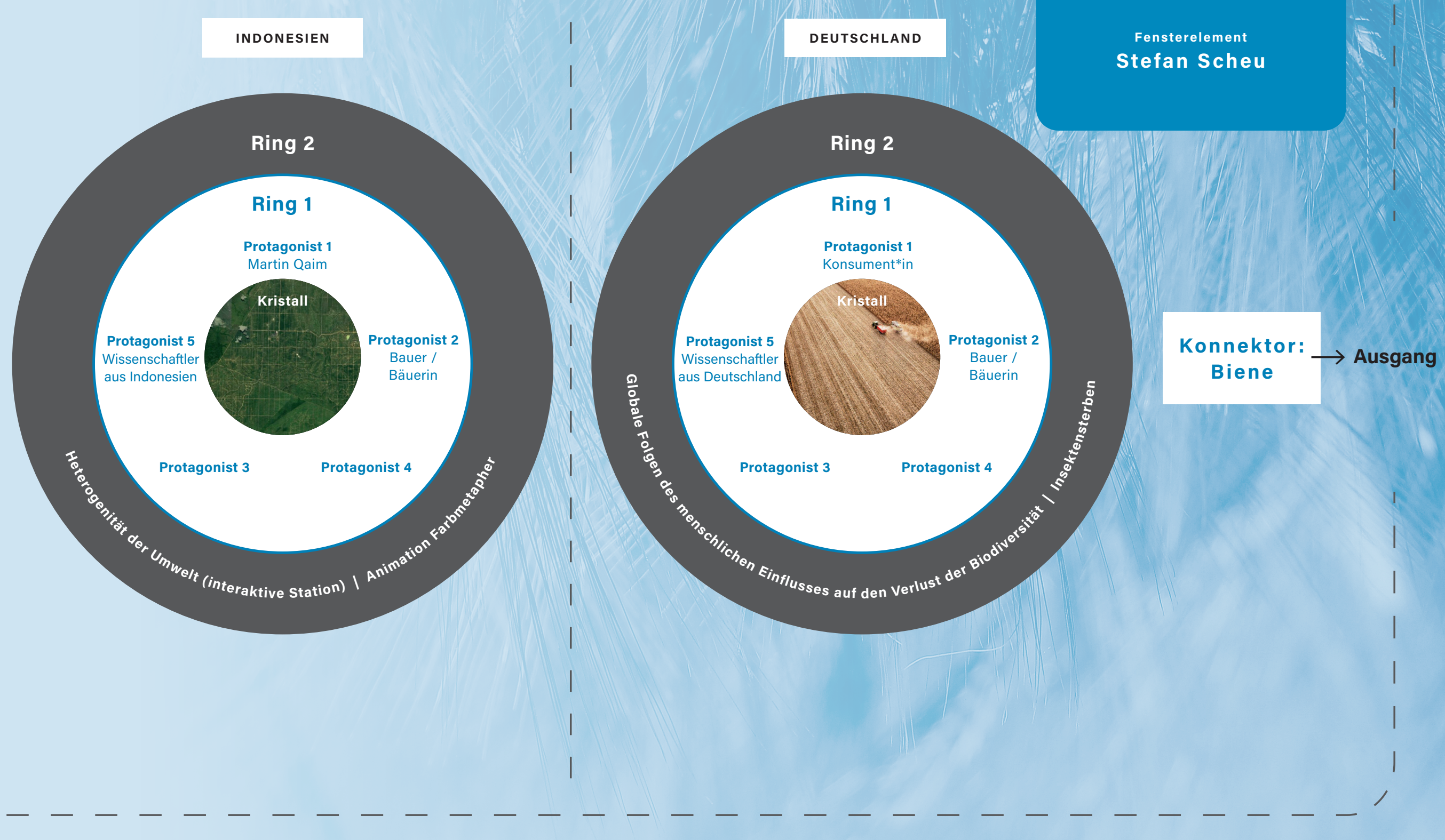




\section{Der Konnektor}

In letzter Zeit hat sich die Zahl der Insekten drastisch verringert, was zum Teil auf den Einsatz von Insektiziden und veränderte Landnutzung zurückzuführen ist.

Wie andere Insekten auch, haben Bienen damit zu kämpfen, in einem von Monokulturen und intensiver Landwirtschaft geprägten Lebensraum genügend Ressourcen zu finden. Am Ende des Kapitels sucht eine einzelne Biene zwischen all den Maispflanzen nach Blüten und fliegt dann in den nächsten Raum, wo sie eine günstigere Umgebung vorfindet. 


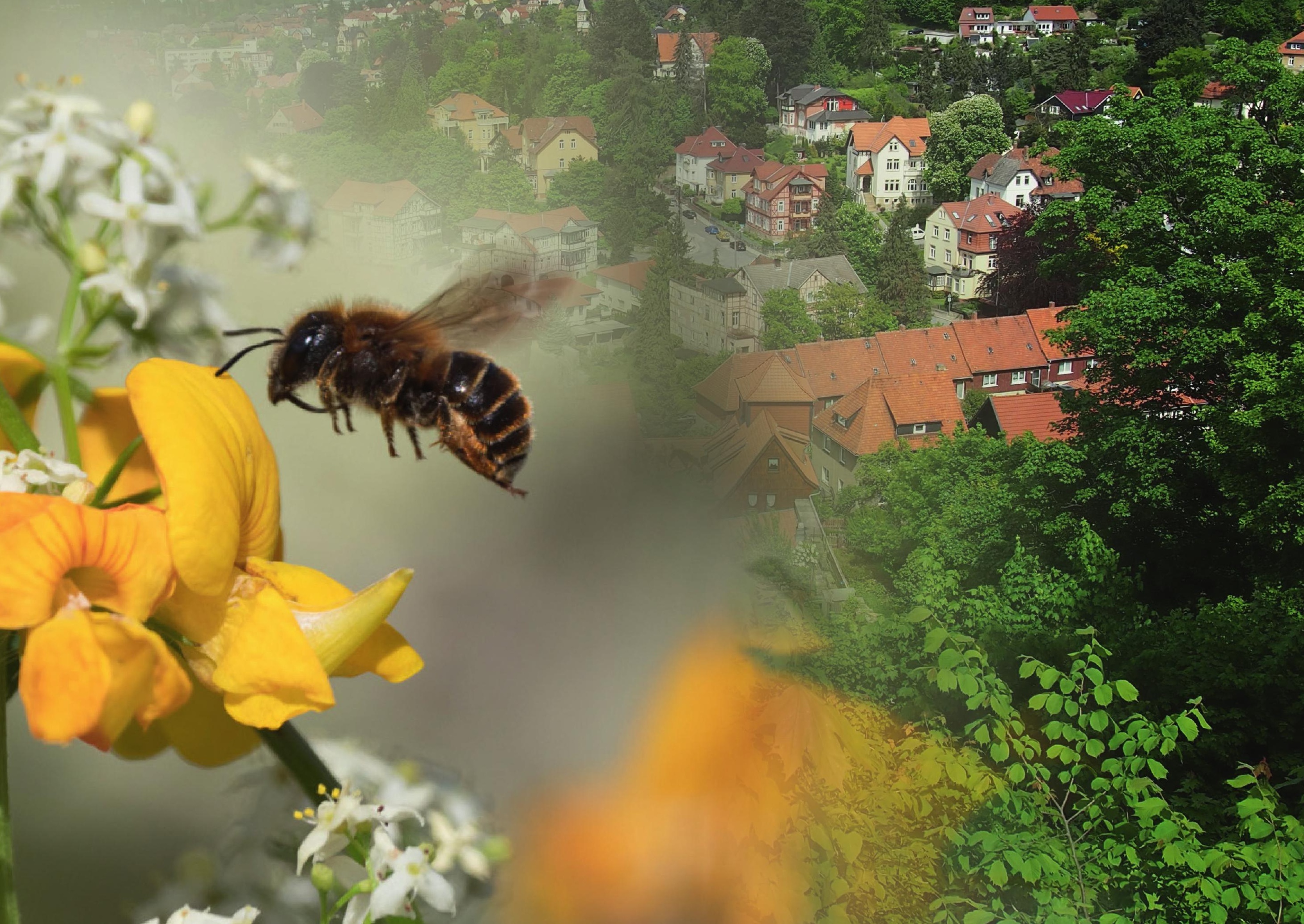




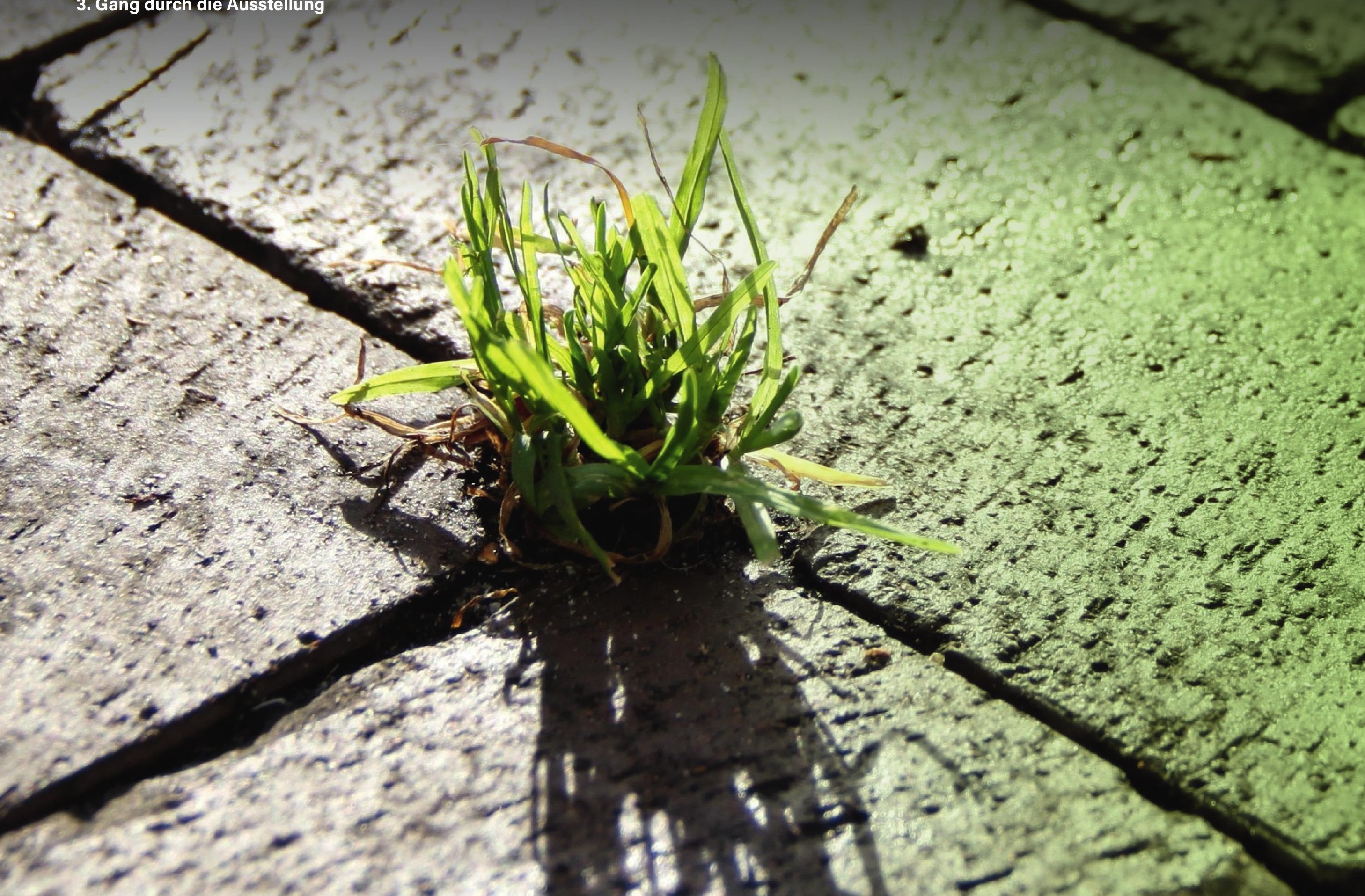




\section{Entdecke}

Biodiversität in deiner Umgebung

«Zugleich glaubt fast jeder Zweite, dass der Verlust der Biodiversität in Deutschland ", kein großes Problem" sei. Das Artensterben vermuten die meisten in den Entwicklungsländern. Bei der Option, im eigenen Garten Raum für seltene Pflanzen und Tiere zu schaffen, kreuzten die meisten dagegen an: „Kommt für mich nicht infrage."»

- Artenschwund im Kopf, GEO 3/10

\section{Kapitel 6}




\section{Szenografie \& Motive}

Im letzten Kapitel sind die Besucher*innen von ihren Erkundungen exotischer Ökosysteme zurückgekehrt. Wieder zu Hause in Göttingen sind sie bereit die Artenvielfalt in ihrer Umgebung zu entdecken.

Dazu gehören Naturschutzgebiete wie der Nationalpark Harz und das Grüne Band, die eine hohe Biodiversität aufweisen. Außerdem werden regionale Erfolgsgeschichten von Arten vorgestellt, die lange Zeit selten oder vollständig verschwunden waren und zurückgekehrt sind, wie der Wolf, der Luchs oder der Biber.

Schauplatz des Kapitels ist ein Friedhof in Göttingen. Obwohl er mitten in der Stadt liegt, ist er vielfältiger und natürlicher als die Monokulturen des letzten Kapitels - es gibt Bäume, Vögel und Insekten, sogar einen Fuchs. Hier lernen die Besucher*innen, dass eine Stadt eine viel gröBere Biodiversität haben kann als ländliche Regionen eine überraschende Tatsache für die meisten Menschen. Auch wird gezeigt, wie man in seiner näheren Umgebung auf Biodiversität achten kann und sie, wann immer möglich, fördern kann.

Der Kristall: Schlüsselexemplare

Regionale Erfolgsgeschichten von zurückgekehrten Arten: der Wolf, der Luchs, der Biber etc.

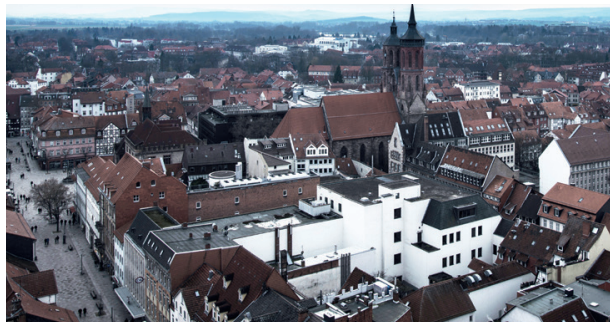

Göttingen

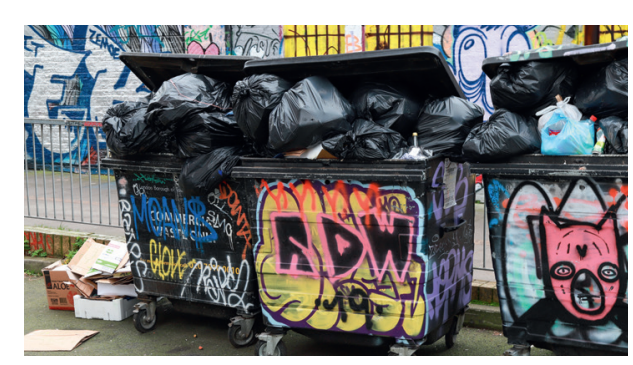

Müllcontainer

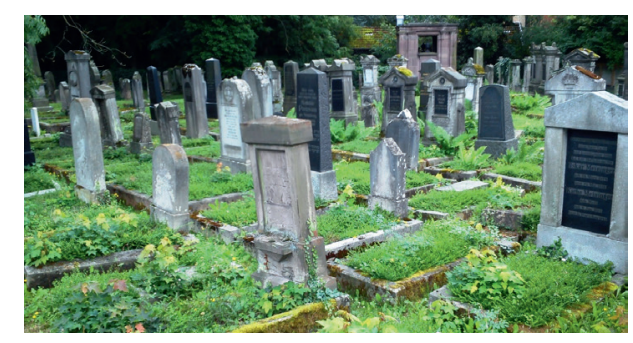

Friedhof

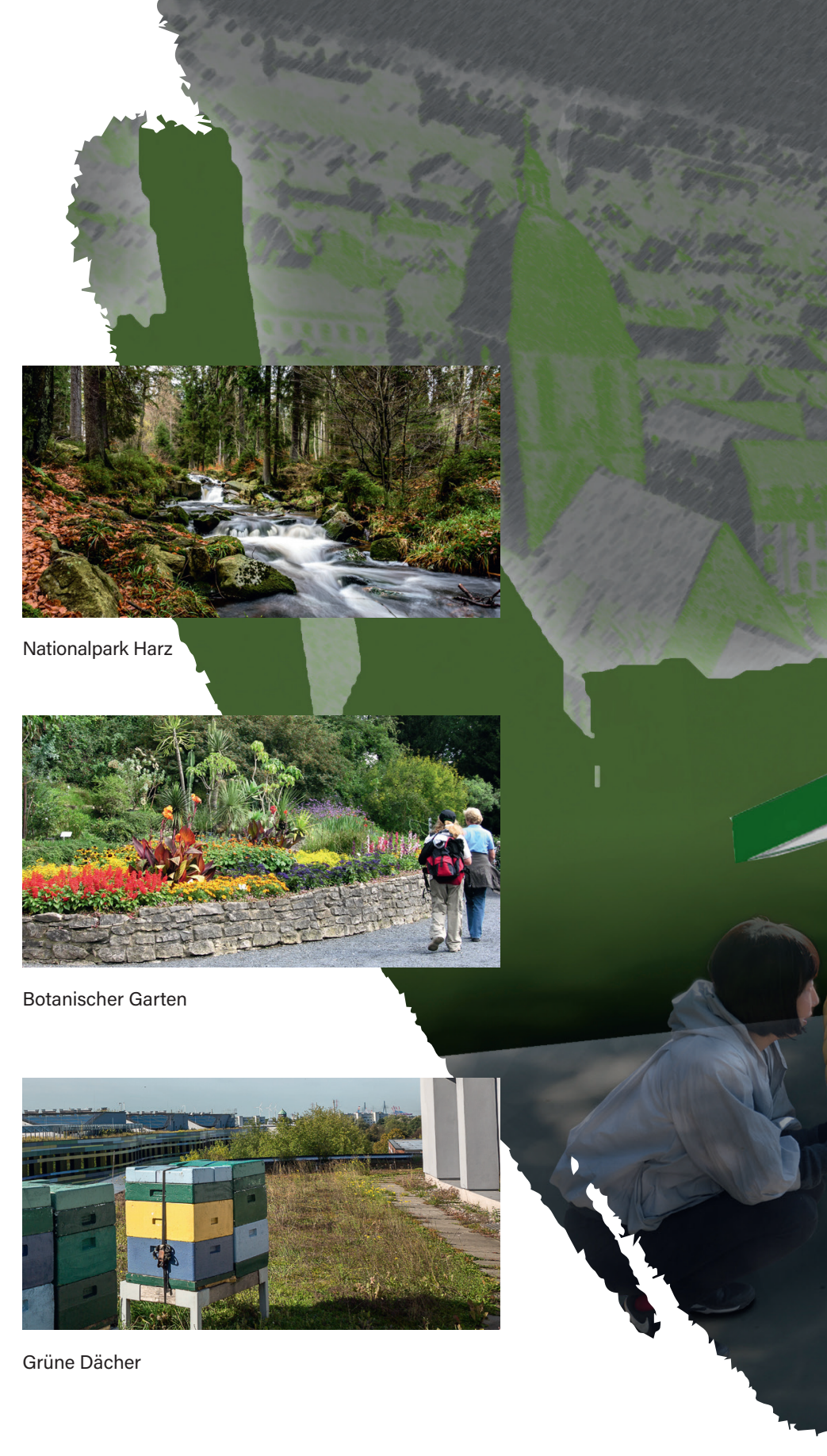




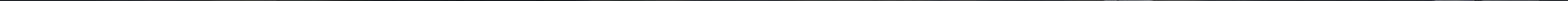




\section{Themen \& Interaktionen}

Ring 1: interaktive Stationen

- Wilde Tiere in den Stadtgebieten

- Verschiedene Arten von Insekten in unterschiedlich gestalteten Gärten

- Missverständnisse über städtische Ökosysteme

- Grünes Netzwerk

- Friedhöfe

\section{Ring 2: interaktive Stationen}

- Migration von Tieren aus anderen Lebensräumen in die Stadt (Fuchs, Wildschwein, Ringeltaube)

- Anpassung von Tieren an städtische Ökosysteme (Stadttauben, Amseln, Spatzen etc.)

- Eine Interaktion, die zeigt, wie sich der Individuenreichtum von Arten und die Artenvielfalt während der Umwandlung eines komplexen Ökosystems in andere, einfachere Ökosysteme (bspw. Monokulturen) verändert.
Ring 3: Fensterelemente

- Grünes Band - die ehemalige innerdeutsche Grenze, heute "das längste Naturschutzgebiet" in Deutschland (1300 km)

- Nationalpark-Interview mit einem/einer Ranger/Rangerin

- Naturpark Solling-Vogler

- Städtebauliche Projekte

\section{Tiny Explorers:}

Einfaches Quiz, bei dem Kinder erraten können, welche Tiere in der Stadt und welche auf dem Land leben. Ein Quiz, das zeigt, warum manche Blumen von Käfern, manche von Schmetterlingen und manche von Bienen bevorzugt werden.
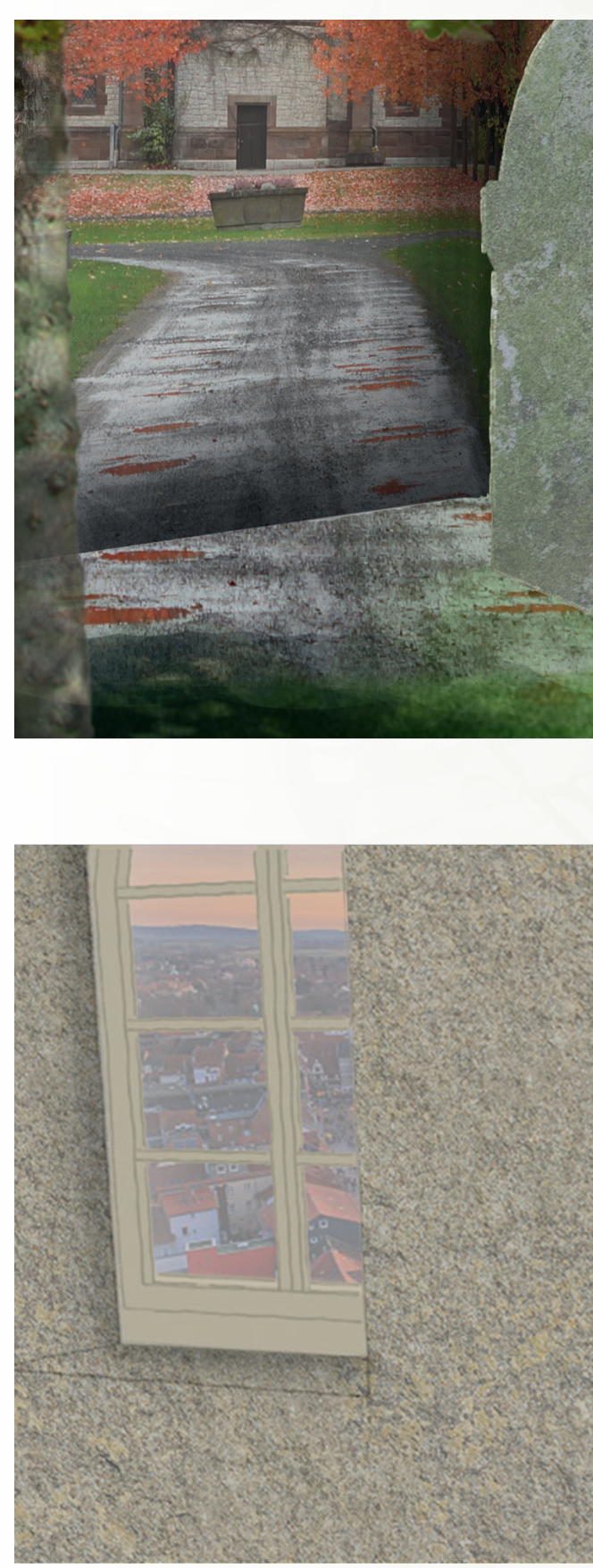

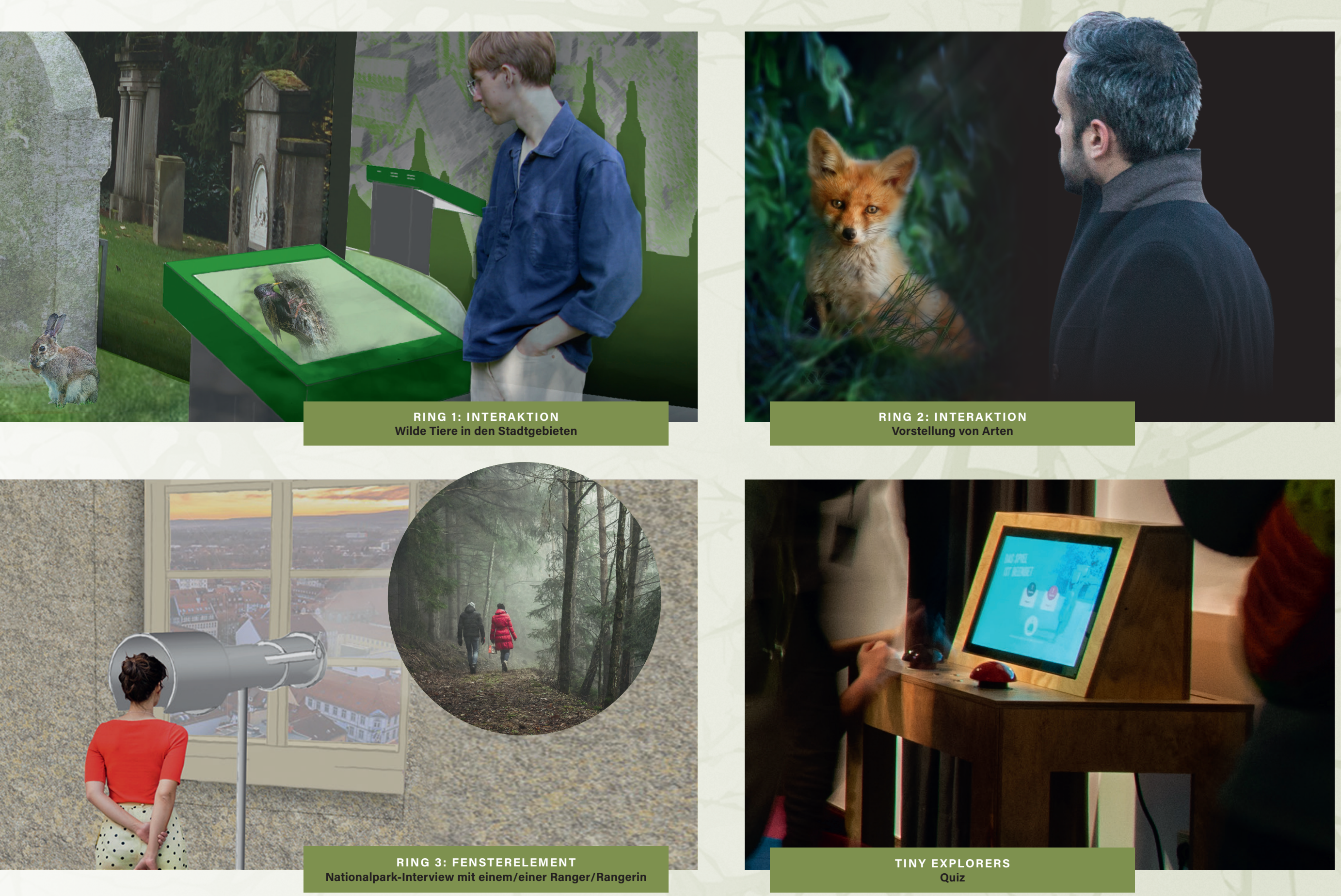


\section{Räumliche Aufteilung}

Prof. Dr. Christoph Leuschner

Christoph Leuschner ist seit 2000 Professor für Pflanzenökologie und Ökosystemfoschung am Albrechtvon-Haller-Institut für Pflanzenwissenschaften. Seine Forschungsschwerpunkte sind unter anderem Ökophysiologie von Bäumen gemäßigter und tropischer Zonen, Ökologie von Baumwurzelsystemen, Walddynamikforschung, Biodiversität und Ökosystemfunktion in Wäldern und Biodiversitätserhaltung in Agrarökosystemen.

\section{Prof. Dr. Alexander Knoh}

Alexander Knohl ist seit 2009 Professor und Leiter der Abteilung Bioklimatologie am Büsgen-Institut. Seine wissenschaftliche Arbeit fokussiert sich auf die Bereiche Biosphären-Atmosphären-Interaktion, Kohlenstoff- und Wasserzyklus in terrestrischen Ökosystemen, Mikrometeorologie und Eddy-Kovarianz-Messungen wie auch auf den Bereich prozessbasierende Ökosystemmodellierung. 
Ring 3:

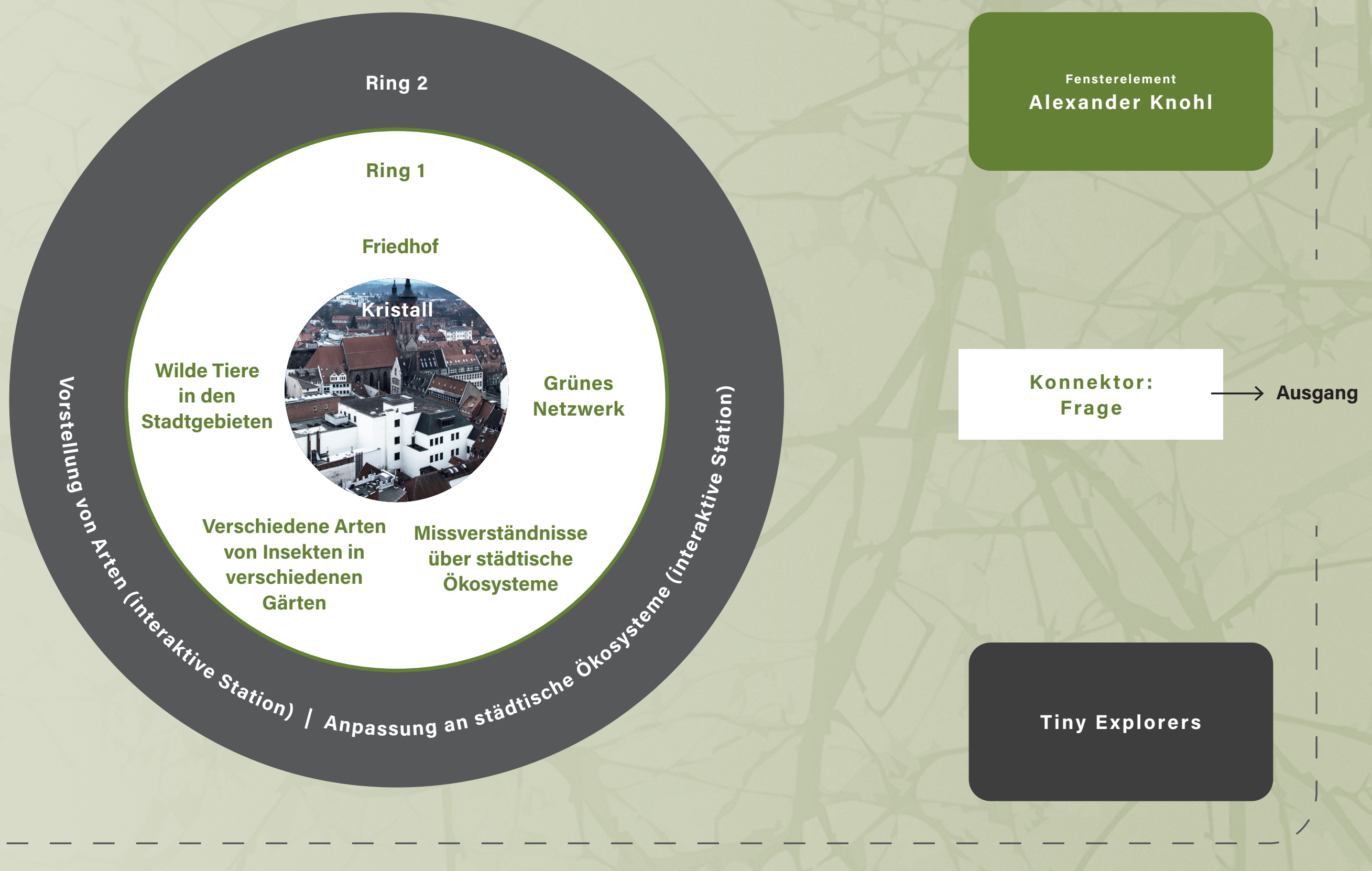




\section{Gang durch die Ausstellung}

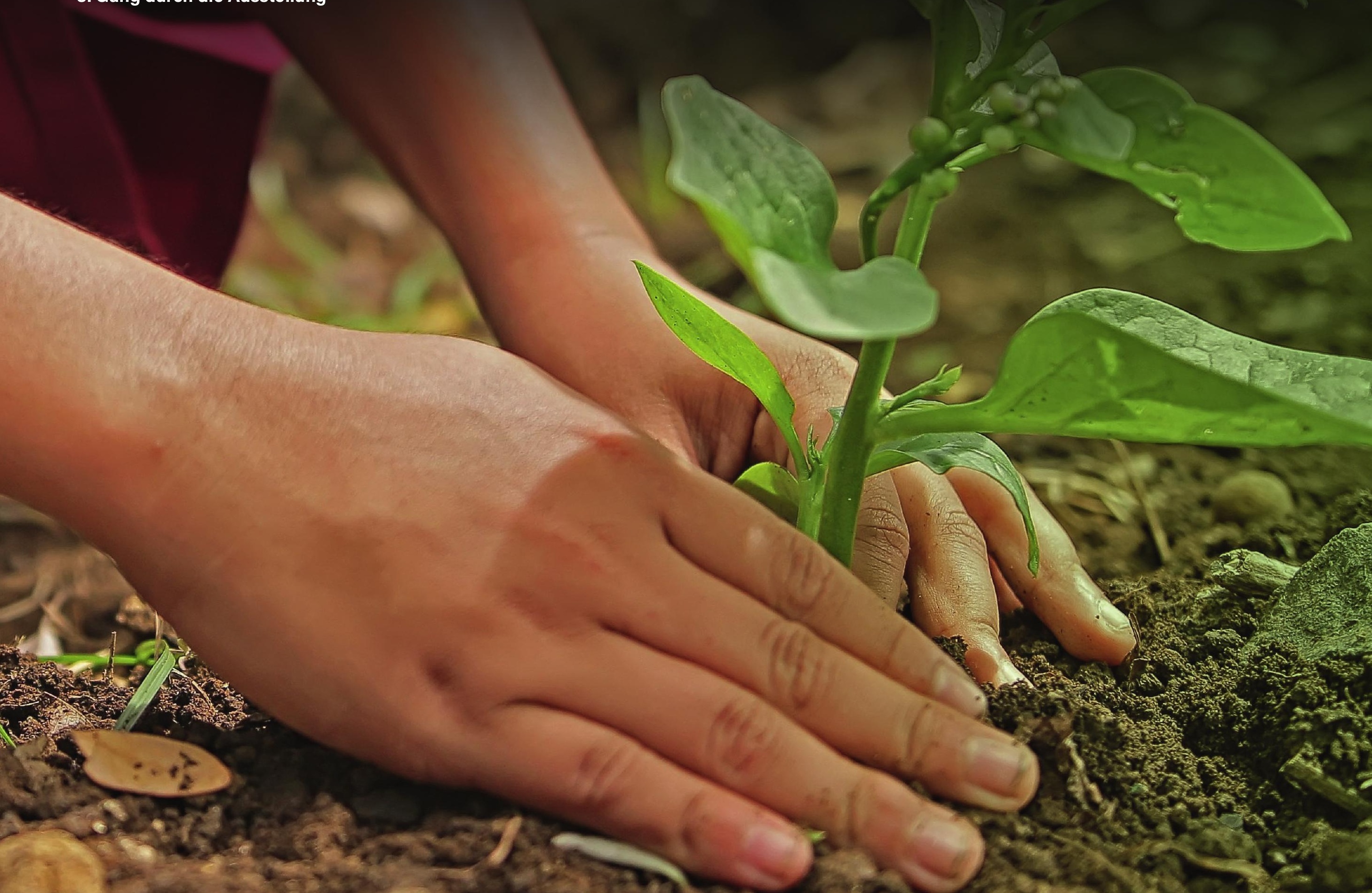




\section{Was kann}

ich tun?

«Dann gäbe es vielleicht eine Antwort auf die Frage, die mir am häufigsten zur Vielfalt des Lebens gestellt wird: Wenn genug Arten ausgelöscht wurden, bricht dann das Ökosystem zusammen, und folgt das Aussterben der meisten anderen Arten bald darauf? Die einzige Antwort, die man geben kann, ist: möglicherweise. Bis wir das herausfinden, könnte es allerdings schon zu spät sein. Ein Planet, ein Experiment.»

- Edward O. Wilson

\section{Der Epilog}




\section{Epilog: Was kann ich tun?}

Nachdem die Besucher*innen nun so viel über Biodiversität erfahren haben, ist es Zeit zu handeln! Im Epilog finden sie zusätzliche Informationen über Menschen, die sich für den Erhalt der Biodiversität einsetzen, und über Projekte, die gerade entwickelt werden.

Die Besucher*innen können sich auch über ihren eigenen ökologischen Fußabdruck und über Möglichkeiten des Handelns informieren. Schließlich können sie Wünsche oder Versprechen auf einen Zettel schreiben und diesen an den "Baum der Wünsche" hängen, damit auch andere Besucher*innen ihn lesen können. Nach der Reise durch die verschiedenen Kapitel sind die Besucher idealerweise in der Lage, auf die gleichen Fragen, die innen am Anfang gestellt wurden, komplexere Antworten zu geben. Hoffentlich sind innen auch die aktuellen Umweltprobleme und der Verlust der Biodiversität bewusster. Es wird Monitore geben, die die Antworten vorheriger Besucher*innen zeigen.

Zusätzlich zu diesen Fragen wird es weitere geben, die sich mehr darauf beziehen, was wir in unserem täglichen Leben tun können, um zu einer nachhaltigeren Zukunft beizutragen.

\section{Der Kristall:}

Baum der Wünsche

\section{Themen:}

- Wie können wir mehr über Biodiversität lernen (Beispiel: App „Flora Incognita")?

- Wie können wir dazu beitragen, das Wissen über unsere lokale Tier- und Pflanzenvielfalt auszubauen (Beispiel: Citizen-Science-Projekt „Stunde der Gartenvögel")?

- Was können wir in unserem täglichen Leben tun, um die Biodiversität zu erhalten, sowohl global als auch zu Hause?

- Wo können wir unseren Einfluss als Bürger nutzen?

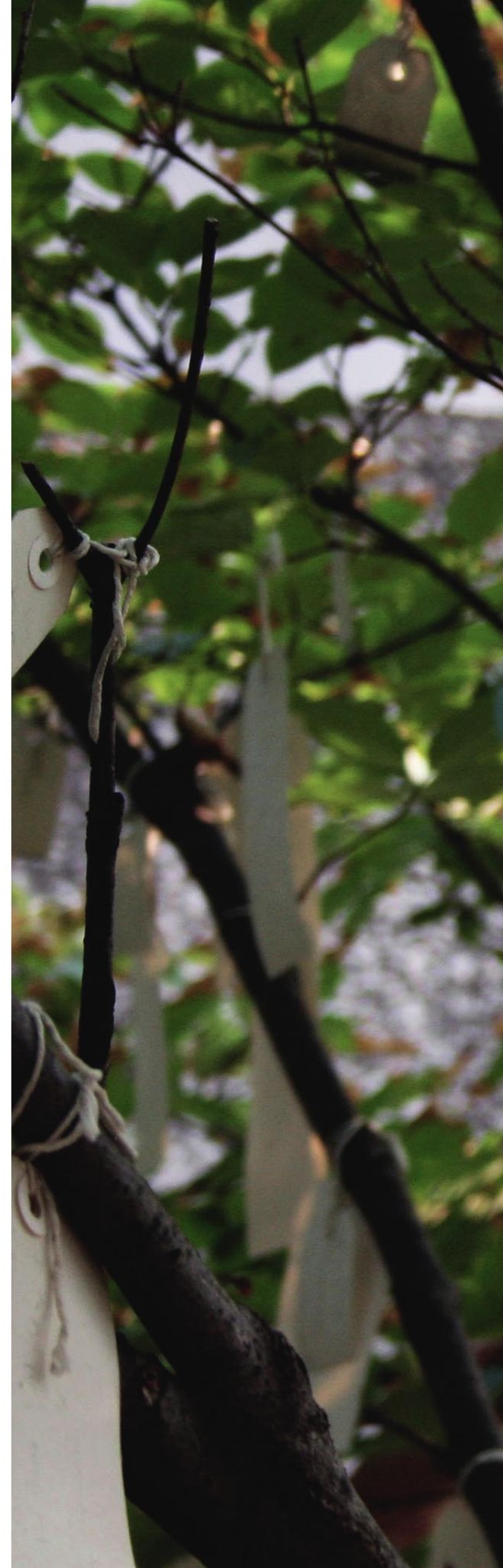




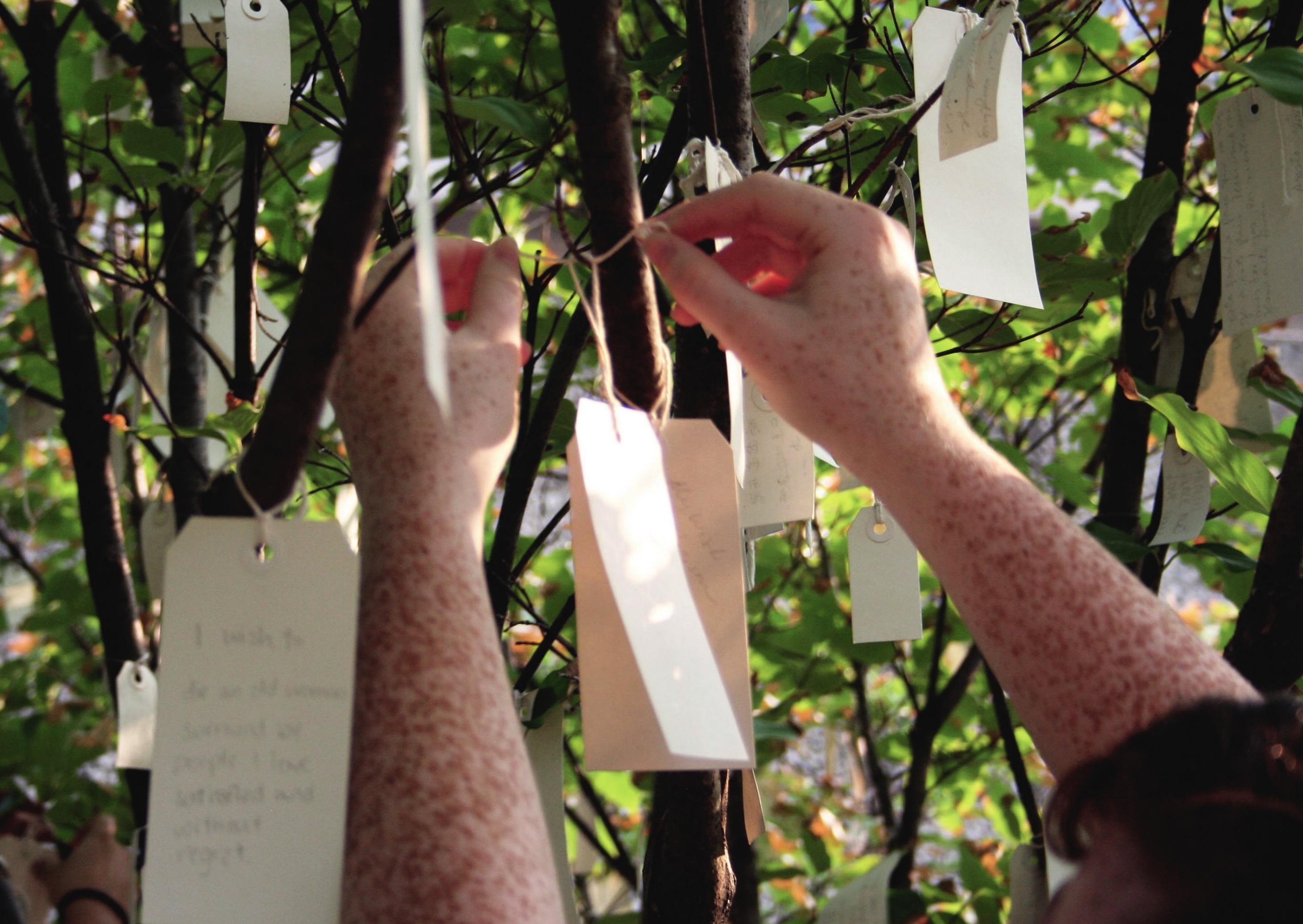




\section{Räumliche Aufteilung}

\section{Dr. Simone Pfeiffer}

Simone Pfeiffer ist seit 2013 wissenschaftliche Koordinatorin für tropische und subtropische Agrar- und Forstwissenschaften im Zentrum für Biodiversität und Nachhaltige Landnutzung. Ihre Arbeit beinhaltet die Antragstellung und Administration interdisziplinärer Verbundprojekte in Forschung und Lehre, Alumniarbeit und Veranstaltungsmanagement. Sie studierte Biologie und promovierte an der Universität Jena.

Fensterelement 
Ring 3:

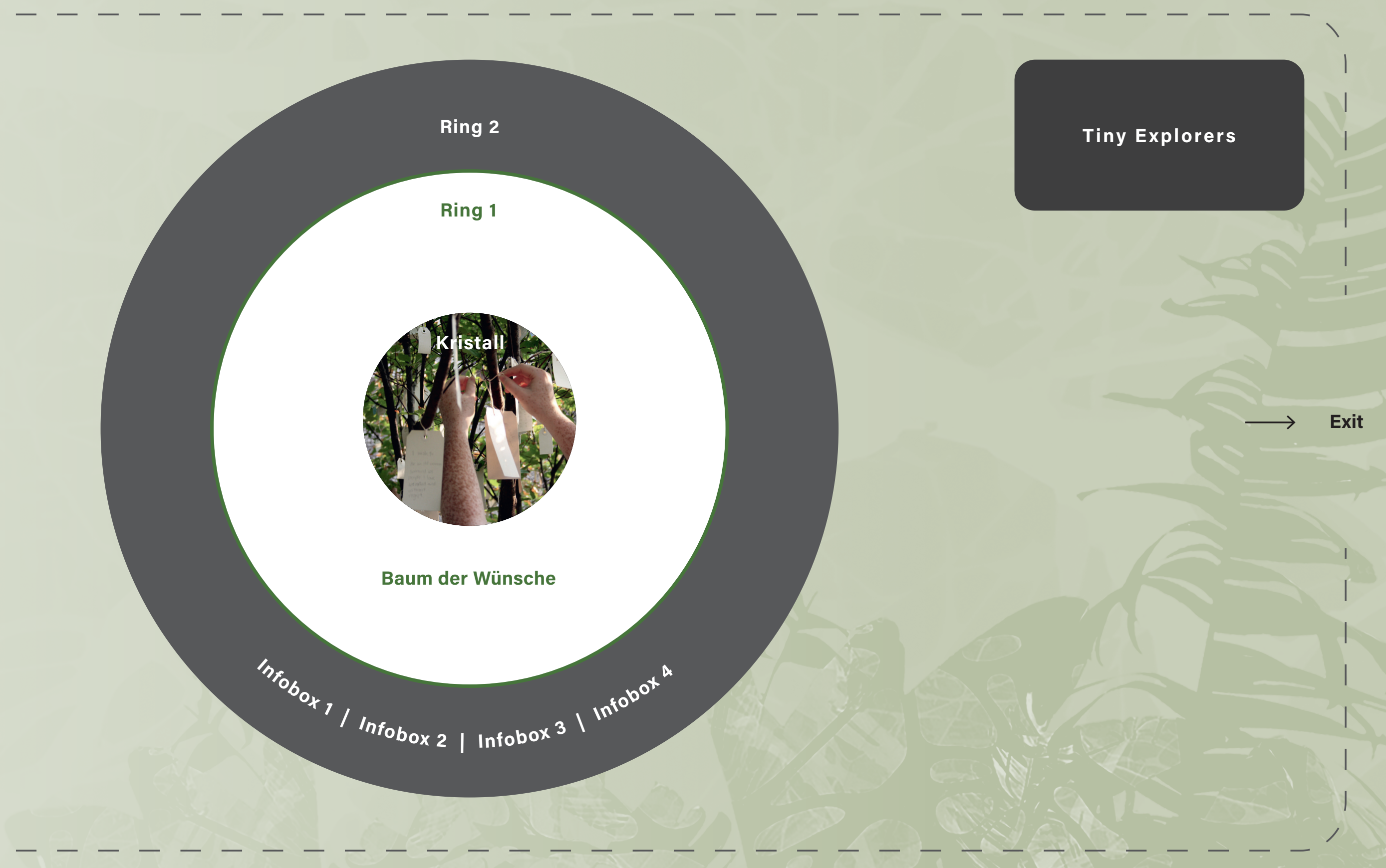


«Es spielt keine große Rolle, ob es den Leuten egal ist oder nicht. Was zählt, ist, dass die Menschen die Welt verändern,»

- Elizabeth Kolbert 


\section{Das Team}

Team des Biodiversitätsmuseums

\section{Göttingen}

Dr. Maria Teresa Aguado Molina

Kuratorin, Projektleiterin des Konzeptdesigns

\section{Prof. Dr. Christoph Bleidorn}

Direktor

\section{Gisa Heinemann}

Sammlungsverwaltung und Didaktik

\section{Monika Endres}

Sammlung und Design

\section{Gerhard Hundertmark}

Fotograf

\section{Carsten Wortmann}

Präparator

\section{Dr. Gert Tröster}

Kurator Zool. Museum (im Ruhestand)

Gisela Vogel

Technische Mitarbeiterin (im Ruhestand)

Illustrationen von Ana Sanz Duran
Wissenschaftlicher Beirat

an der Universität Göttingen

\section{Prof. Dr. Hermann Behling}

Fakultät für Biologie \& Psychologie

\section{Dr. Sven Bradler}

Fakultät für Biologie \& Psychologie

\section{Prof. Dr. Julia Fischer}

Fakultät für Biologie \& Psychologie

Prof. Dr. Thomas FriedI

Fakultät für Biologie \& Psychologie

\section{Prof. Dr. Alexander Knoh}

Fakultät für Forstwissenschaften \& Waldökologie

\section{Prof. Dr. Holger Kreft}

Fakultät für Forstwissenschaften \& Waldökologie

\section{Prof. Dr. Christoph Leuschner}

Fakultät für Biologie \& Psychologie

\section{Dr. Maike Lorenz}

Fakultät für Biologie \& Psychologie

\section{Dr. Simone Pfeiffer}

Fakultät für Forstwissenschaften \& Waldökologie

\section{Prof. Dr. Matin Qaim}

Fakultät für Agrarwissenschaften

Prof. Dr. Stefan Scheu

Fakultät für Agrarwissenschaften

Prof. Dr. Catrin Westphal

Fakultät für Agrarwissenschaften
Agentur für Ausstellungsdesign Kunstraum GfK

Friedo Meger

Geschäftsführer

\section{Maria Negrao}

Projektmanagement,

Ausstellungsgestaltung

Melanie Ramsay

Innenarchitektur

\section{Sonja Vogt}

Recherche, Texte und

redaktionelle Arbeit

\section{Alexander Boz}

Ausstellungsgestaltung

\section{Andreas Voigt}

Innenarchitektur,

Lichtdesign

Boris Meise

Industriedesign

\section{Lisa Walter}

Ausstellungsgestaltung

\section{Max Glimm}

Kommunikationsdesign 
«Die Natur kann bewahrt, wiederhergestellt und nachhaltig genutzt werden, während gleichzeitig andere globalgesellschaftliche Ziele durch dringende und konzertierte Anstrengungen erreicht werden, die einen transformativen Wandel fördern.»

- IPBES global assessment report on biodiversity and ecosystem services

\section{Impressum}

Bibliografische Information der Deutschen Nationalbibliothe

Die Deutsche Nationalbibliothek verzeichnet diese Publikation in der Deutschen Nationalbibliografie; detaillierte bibliografische

Daten sind im Internet über http://dnb.dnb.de abrufbar.

Unser Dank gilt der Lindemann-Stiftung, Stiftung de

Georg-August-Universität Göttingen, für ihre großzügige Unterstützung.

Herausgeber*innen: Maria Teresa Aguado Molina, Christoph Bleidorn

Georg-August-Universität Göttingen

Evolution und Biodiversität der Tiere

Untere Karspüle 2

D-37073 Göttingen

Dieses Buch ist auch als freie Onlineversion über die Homepage

des Verlags sow unber den Gottinger Universitatskatalog (GUK)

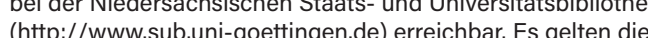

(http://www.sub.uni-goettingen.de) erreicht
Konzept: Maria Teresa Aguado Molina, Christoph Bleidorn, Maria Negrao, Sonja Vogt, Friedo Meger

Text: Maria Teresa Aguado Molina, Christoph Bleidorn, Sonja Vogt

Bearbeitung: Maria Teresa Aguado Molina, Christoph Bleidorn Monika Endres, Gert Tröster, Sonja Vogt

Design: Max Glimm, Maria Teresa Aguado Molina,

Monika Endres, Ana Sanz Duran, Maria Negrao

Visualisierungen: Maria Negrao, Melanie Ramsay

Fotografie: Gerhard Hundertmark

○ 2021 Göttingen University Press/

Universitätsverlag Göttingen

http://univerlag.uni-goettingen.de

ISBN: 978-3-86395-487-1

DOI: https://doi.org/ 10.17875/gup2021-1581
GEORG-AUGUST-UNIVERSITÄT GÖTTINGEN

Georg-August-Universität Göttingen

Wilhelmsplatz 1, 37073 Göttingen

www.uni-goettingen.de

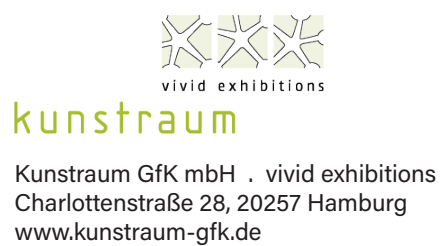




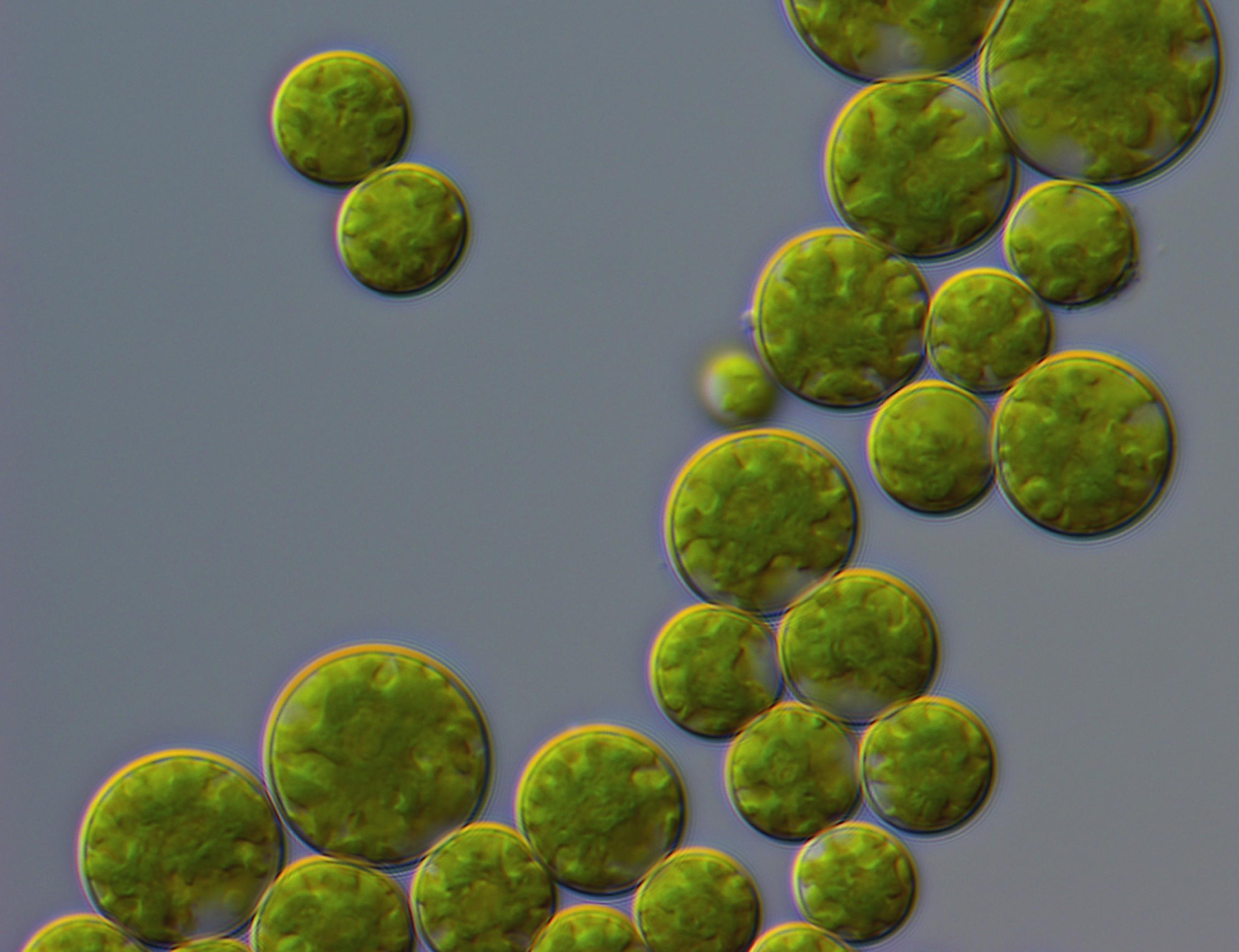




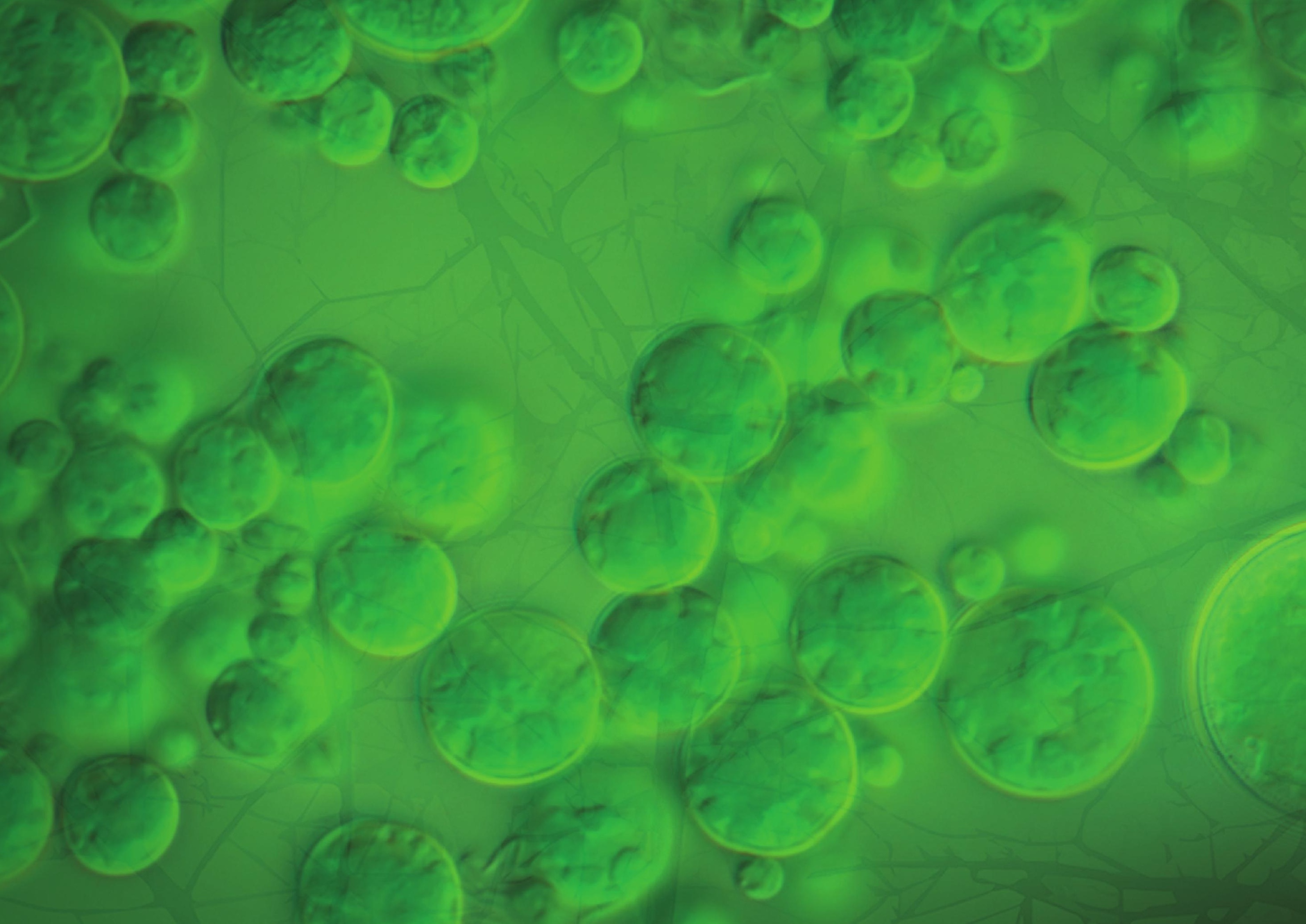

